\title{
INVESTIGATION OF THERMODIFFUSION PHENOMENON UNDER INFLUENCE OF VIBRATION USING IMAGE PROCESSING APPROACH
}

\author{
By \\ Armin Kianian \\ B.Sc. in Mechanical Engineering, Ryerson University, Toronto, Canada, 2010
}

\author{
A thesis \\ presented to Ryerson University \\ in partial fulfillment of the \\ requirement for the degree of \\ Master of Applied Science \\ in the Program of \\ Mechanical Engineering
}

Toronto, Ontario, Canada, 2013

(C) Armin Kianian 2013 


\section{AUTHOR'S DECLARATION}

I hereby declare that I am the sole author of this thesis. This is a true copy of the thesis, including any required final revisions, as accepted by my examiners.

I authorize Ryerson University to lend this thesis to other institutions or individuals for the purpose of scholarly research.

I further authorize Ryerson University to reproduce this thesis by photocopying or by other means, in total or in part, at the request of other institutions or individuals for the purpose of scholarly research.

I understand that my thesis may be made electronically available to the public. 


\title{
ABSTRACT \\ Investigation of Thermodiffusion Phenomenon under Influence of Vibration using Image Processing Approach
}

\author{
Armin Kianian \\ Master of Applied Science \\ Mechanical and Industrial Engineering, 2013 \\ Yeates School of Graduate Studies \\ Ryerson University, Toronto, ON, M5B 2K3, Canada
}

Thermodiffusion is the appearance of concentration gradient in a mixture when subjected to a temperature gradient. Thermodiffusion or Soret effect plays an important role in the underground reservoir distribution. Consequently, a majority of petroleum research is focused on understanding this phenomenon. There are many experimental measurements of thermodiffusion. However, measurements conducted in a microgravity environment minimize the effect of gravity and leads to accurate results.

This study demonstrates the influence of vibration on thermodiffusion measurement in a microgravity environment. The aim is to show how the variation of certain parameters such as the frequency and amplitude of translational vibration as well as the temperature would impact the composition of components in the mixture of Water-Isopropanol.

The Fast Fourier Transform image processing technique is used to analyse the data obtained from optical digital interferometry. Moreover, two sets of experimental runs with negative and positive Soret coefficients are tested.

The analysis shows the maximum separation of components for the case without any forced vibration. Furthermore, it is shown the increase in Rayleigh number corresponds to decrease in separation of components. 


\section{ACKNOWLEDGEMENTS}

Firstly, I would like to express my deepest gratitude to Dr. Ziad Saghir for giving me the privilege to work under his supervision and also providing me with his support and guidance throughout different stages of my Master program.

I would like to thank the Canadian Space Agency and European Space Agency for funding this work.

I would like to thank my parents and my wife for their never ending supports.

Also, I would like to show my appreciation to my colleagues Mr. Ahadi, Mr. Khoshnevis, and Mr. Abdur Rahman for providing me with insightful information and helpful discussions. 


\section{TABLE OF CONTENTS}

AUTHOR'S DECLARATION ...........................................................................................ii

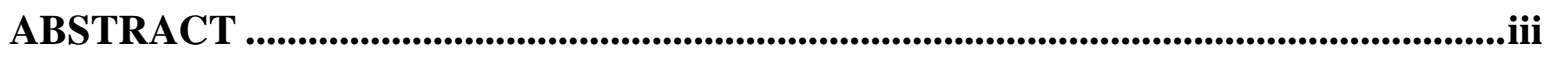

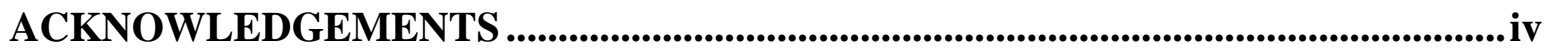

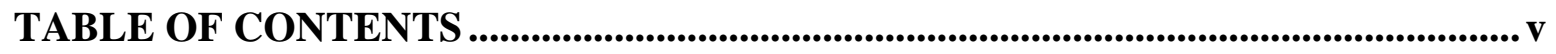

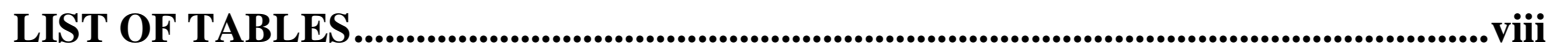

LIST OF FIGURES..............................................................................................................................ix

NOMENCLATURES ..................................................................................................

CHAPTER 1 INTRODUCTION ..............................................................................1 1

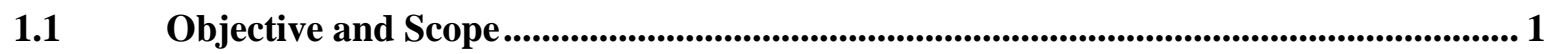

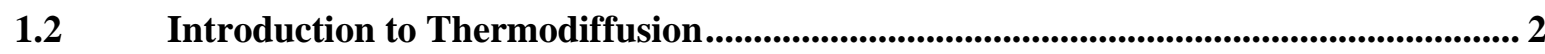

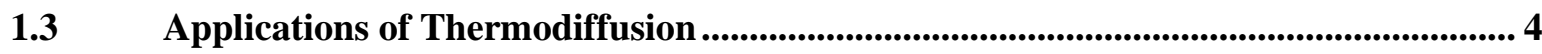

1.4 Experimental Measurements of Thermodiffusion ...............................................................5

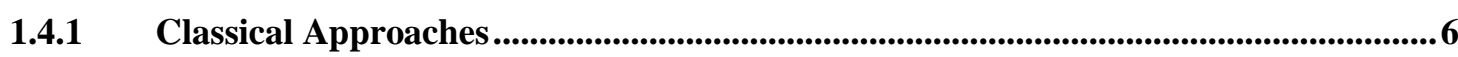

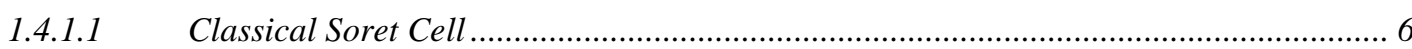

1.4.1.2 Thermogravitational Column (TGC) Technique ….................................................... 8

1.4.2 Optical Techniques ............................................................................................................................. 11

1.4.2.1 Soret Cell With Beam Deflection (BD) Technique ................................................... 12

1.4.2.2 Thermal Diffusion Forced Rayleigh Scattering (TDFRS) ........................................... 14

1.5 Thermodiffusion of Binary Fluid Mixture in Microgravity ............................................ 16

1.5.1 Microgravity Experiment............................................................................................. 18

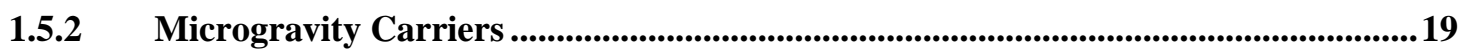

1.5.2.1 ～Drop Tower, Drop Tubes, Sounding Rockets ............................................................. 19

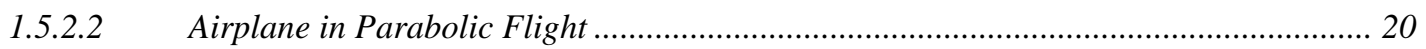

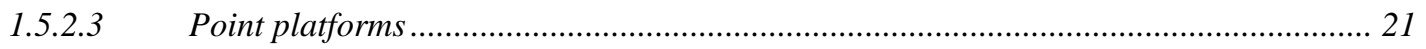

1.5.3 Disturbance in zero-g conditions .................................................................................................22 


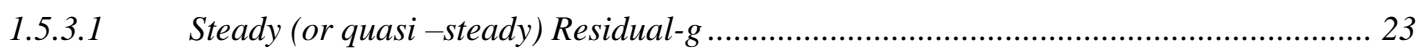

1.5.3.2 Pulses-like (single or compensating) Accelerations ................................................... 23

1.5.3.3 Periodic High Frequency Acceleration ................................................................ 23

1.5.4 Experiments to Study the Impact of Residual Acceleration .........................................25

1.5.4.1 On aboard the orbital station "MIR" by the convection sensor "DACON"................ 25

1.5.4.2 MEPHISTO data from USMP-3 space flight mission........................................... 26

1.5.4.3 Influence of $g$-jitter on solute diffusion coefficient on-board the MIR station.............. 26

1.5.4.4 IVIDIL Experiment on board ISS ......................................................................... 26

CHAPTER 2 SPACE EXPERIMENTAL SETUP ..................................................29

$2.1 \quad$ Pre-flight, In-flight, and Post-flight Activities ................................................................ 29

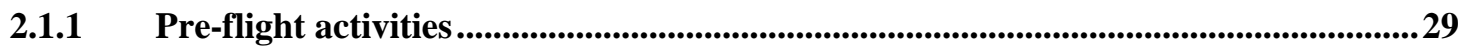

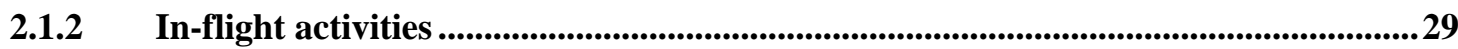

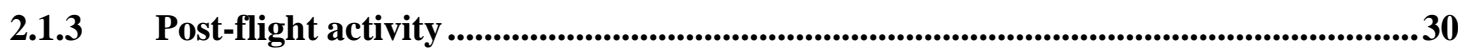

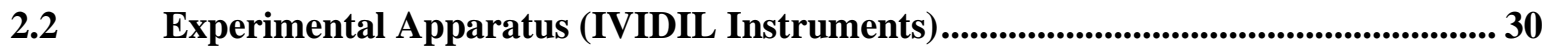

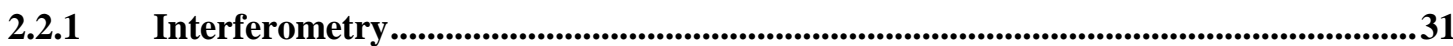

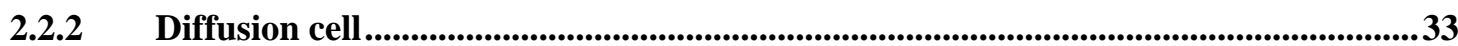

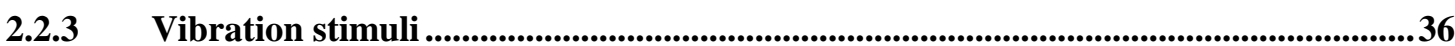

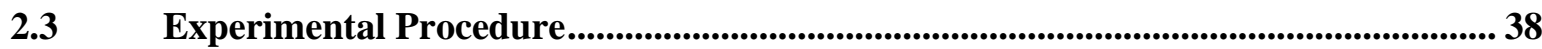

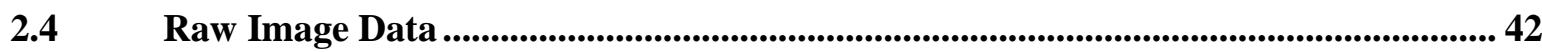

CHAPTER 3 IMAGE PROCESSING TECHNIQUE ..........................................45

Phase Extraction from Fringe Image ................................................................................. 45

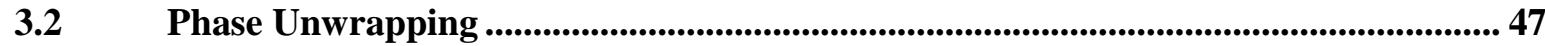

Calculation of Change in Refractive Index ...............................................................50

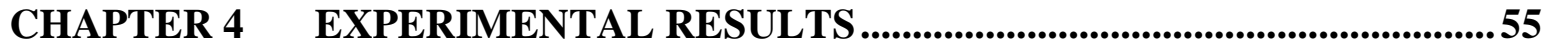

4.1 Analysis of Mixture with Negative Soret Coefficient .................................................55

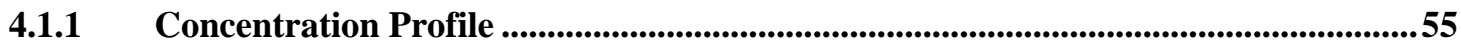

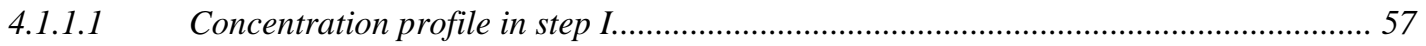




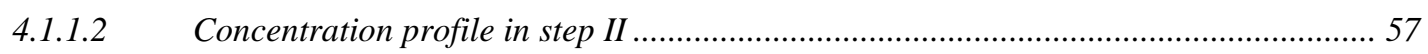

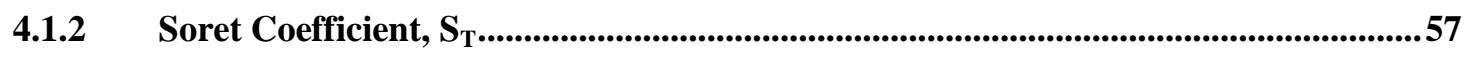

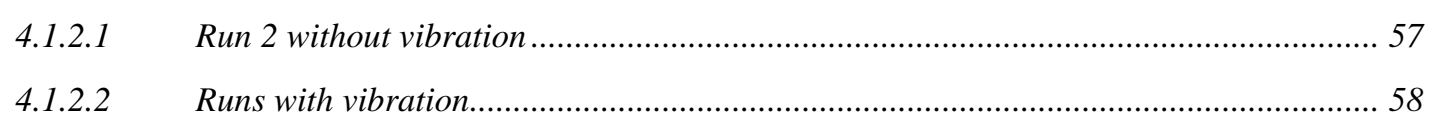

4.1.3 Frequency and Amplitude of Low Rayleigh Vibration ............................................58

4.1.3.1 Effect of frequency variation on Soret coefficient measurement ................................ 58

4.1.3.2 Effect of amplitude variation on Soret coefficient measurement ............................... 59

4.1.4 Temperature Distribution in the Experimental Cavity .......................................60

4.1.5 Concentration Distribution in the Experimental Cavity ......................................62

4.2 Analysis of Mixture with Positive Soret Coefficient................................................... 64

4.2.1 Analysis of Run 34 Results ................................................................................65

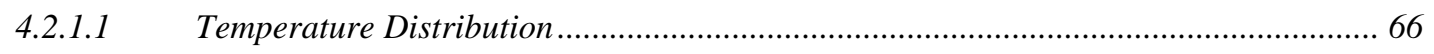

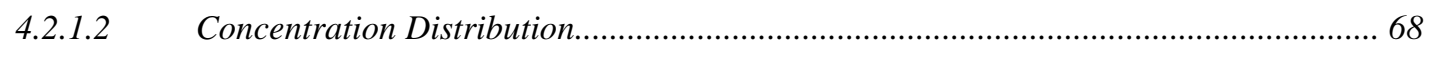

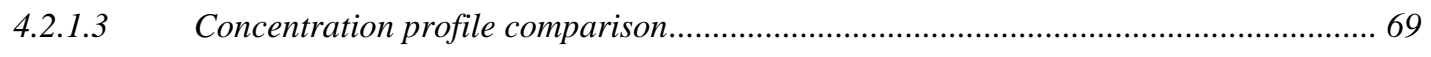

4.2.2 Analysis of Run 36 Results ..............................................................................70

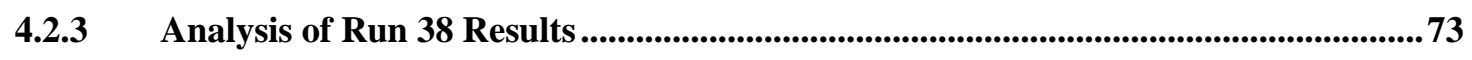

4.2.4 Analysis of Run 32 Results ...................................................................................... 74

CHAPTER 5 CONCLUSION AND RECOMMENDATIONS ................................78

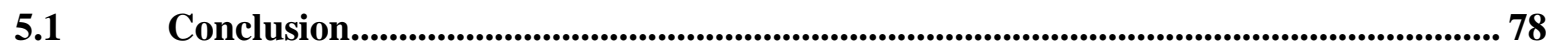

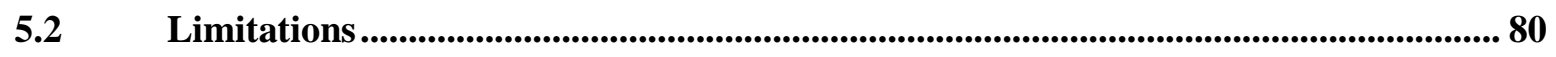

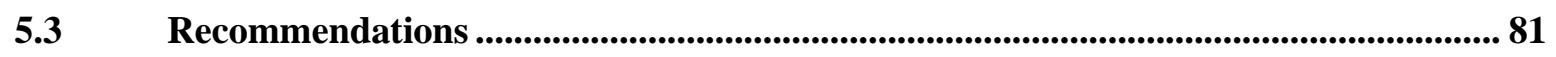

PUBLICATIONS.............................................................................................................................8

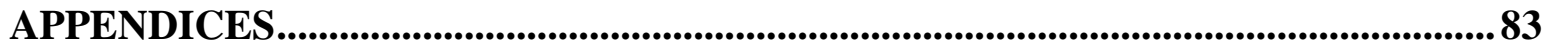

Appendix A: MATLAB code to generate phase difference ....................................................83

Appendix B: MATLAB code to generate concentration difference ..................................884

BIBLIOGRAPHY .................................................................................................................86 


\section{LIST OF TABLES}

Table 1-1: Characteristics of microgravity carriers [33] .............................................. 22

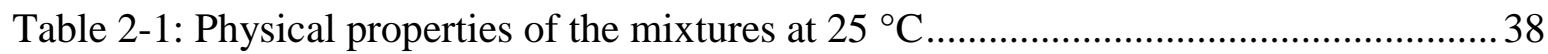

Table 2-2: Selected IVIDIL runs and properties ........................................................ 41

Table 2-3: Description of raw image naming convention [49] .................................... 43

Table 3-1: Variation of refractive index for Water-IPA [53] ........................................51

Table 4-1: Comparison of $\Delta \mathrm{C}$ and $\mathrm{S}_{\mathrm{T}}$ for ISS experimental runs at steady state condition .56

Table 4-2: Comparison of $\Delta \mathrm{C}$ and $\mathrm{S}_{\mathrm{T}}$ for ISS experimental runs at steady state condition . 77 


\section{LIST OF FIGURES}

Figure 1-1: 2-D Model of thermodiffusion cell [4] ..................................................... 2

Figure 1-2: Schematic of a classical Soret cell [6] ..................................................... 6

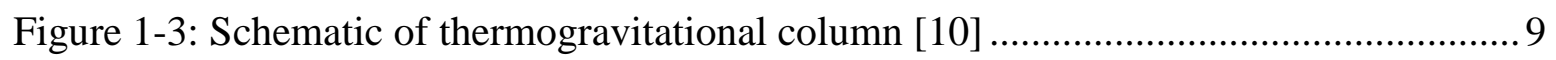

Figure 1-4: Schematic of beam deflection apparatus. A and B are the initial and steady state points of incidence of laser beam on the screen [6] ................................................... 12

Figure 1-5: Schematic of thermal diffusion forced Rayleigh scattering setup [24] ............ 15

Figure 1-6: Transient Soret separation, $S_{R}$ at different static gravity levels, $\nabla \mathrm{T} \perp \mathrm{g}[28] \ldots .17$

Figure 1-7: Dependence of the steady state Soret separation on the gravity level [28] ....... 17

Figure 1-8: Schematic view of the trajectory of parabolic flights [33] ............................ 21

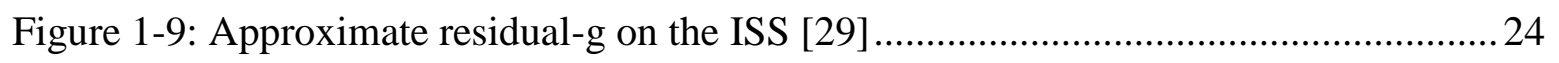

Figure 2-1: IVIDIL inside Glovebox at the ISS [45] ............................................. 31

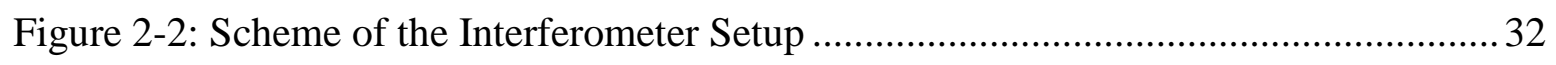

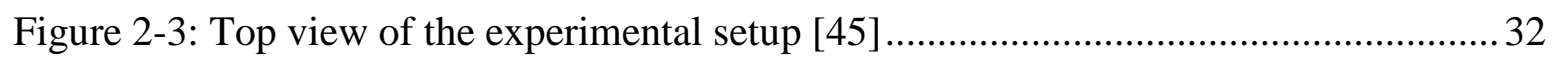

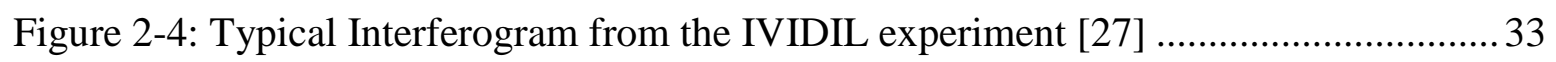

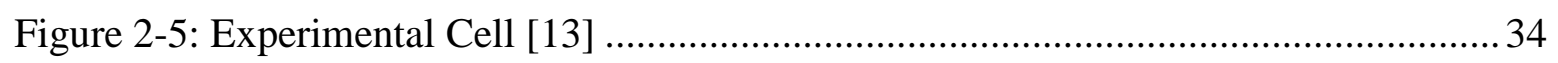

Figure 2-6: The vertical temperature profiles: inside glass wall, inside liquid (water) and expected linear behaviour [47] ........................................................................ 34

Figure 2-7: Cubical cell and core part of IVIDIL instrument; by courtesy of Verhaert Space

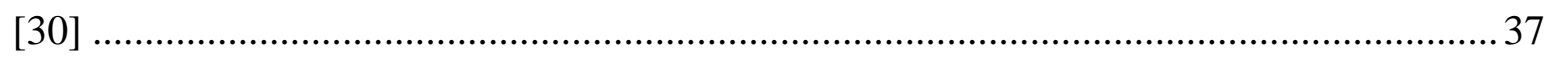

Figure 2-8: Canadian Space Agency Astronaut Bob Thirsk prepares the cell array [45] ....39

Figure 2-9: Concentration profile for Run 2 IVIDIL [46] .......................................... 40

Figure 2-10: Image data after converting from RAW to BMP format [49] ...................... 42

Figure 3-1: Principle scheme of image processing steps (A: Crop the original image to remove wall effect, B: 2D Fast Fourier Transform (FFT), C: Filter out one of the non-zero peaks and bring it in the middle using adjustable $[\mathrm{x}, \mathrm{y}]$ filter mask, D: Inverse $2 \mathrm{D}$ FFT, E: 
Subtracting the image from reference image, F: Unwrapping the subtracted image, G:

Contour of refractive index, $\mathrm{H}$ : Contour of Temperature and Concentration)

Figure 3-2: Variation of refractive index as function of mass fraction of water. Open circles correspond to laboratory measurements [52] 52

Figure 3-3: Evolution of the phase difference with time during thermodiffusion experiment. [52] 53

Figure 4-1: Concentration profile at different time for low Rayleigh vibration runs..........56

Figure 4-2: Temperature distribution in the cavity during thermal time. Run 1 ...............61

Figure 4-3: Temperature distribution in the cavity during thermal time. Run 2 ...............61

Figure 4-4: Temperature distribution in the cavity during thermal time. Run 3 ..............61

Figure 4-5: IPA concentration profile in the cavity at different time during diffusion time, run1

Figure 4-6: IPA concentration profile in the cavity at different time during diffusion time, run2

Figure 4-7: IPA concentration profile in the cavity at different time during diffusion time, run3

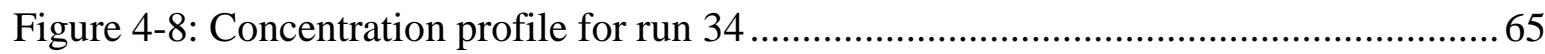

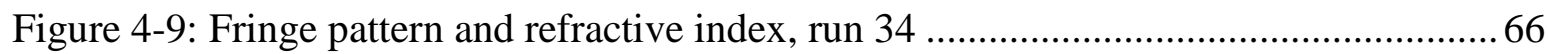

Figure 4-10: Temperature distribution in the cavity during thermal time. Run 34 ...........67

Figure 4-11: IPA concentration profile in the cavity at different time during diffusion time, run34 68

Figure 4-12: Concentration profile for run 34 and run1 comparison ............................. 70

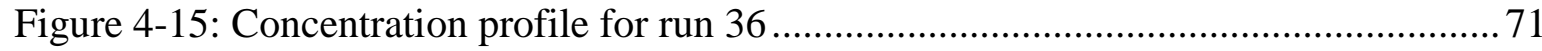

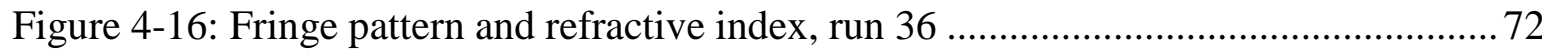

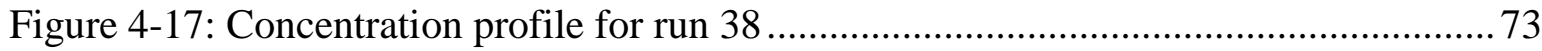

Figure 4-18: Fringe pattern and refractive index, run 38 ......................................... 74

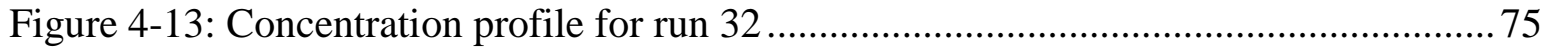

Figure 4-14: Fringe pattern and refractive index, run 32 ............................................ 76 


\section{NOMENCLATURES}

Change in refractive index $\Delta n$

Concentration difference $\Delta C$

Concentration gradient $\quad \nabla c$

Coordinate index of pixel $\quad i, j$

Coordinate system $x, y$

Deflection due to concentration gradient $\Delta \theta_{s}$

Deflection due to thermal gradient $\Delta \theta_{t h}$

Fourier transform of coordinate $u, v$

Gershuni number Gs

Gravitational acceleration $\quad g\left[\mathrm{~m} / \mathrm{s}^{2}\right]$

Height $\quad h[\mathrm{~mm}]$

Initial concentration $\quad c_{o}$

Mass flux $\quad J_{m}\left[\mathrm{~kg} / \mathrm{s} . \mathrm{m}^{2}\right]$

Molecular diffusion coefficient $\quad D\left[m^{2} / s\right]$

Number of pixels in $\mathrm{x}, \mathrm{y}$ direction $m, n$

Optical Length of the cell $\quad L[m m]$

Phase Distribution $\Delta \phi$

Rayleigh vibration $R a_{v i b}$

Refractive index due to temperature

$\left(\frac{\partial n}{\partial T}\right)[1 / K]$

Refractive index due concentration

$\left(\frac{\partial n}{\partial C}\right)$

Separation rate

$S_{R}$

Soret Coefficient

$S_{T}[1 / K]$

Temperature

$T[K]$

Temperature difference $\Delta T[K]$

Temperature gradient

$\nabla T$

Thermal diffusion coefficient

$D_{T}\left[m^{2} / s . K\right]$

Time

$t[s]$

Vibration Amplitude

Vibration frequency

\section{Greek Symbol}

Angular frequency

$\omega[\mathrm{rad} / \mathrm{s}]$

Density of the fluid

$\rho\left[\mathrm{kg} / \mathrm{m}^{3}\right]$

Interference phase

Laser Wavelength 
Relaxation time

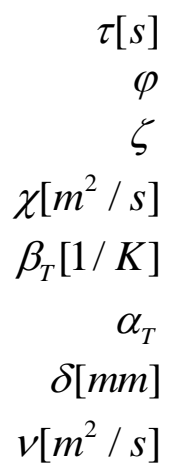

Subscript

Cold

C

Diffusion

D

Hot

$\mathrm{H}$

Reference

ref

Steady

Thermal

st

th 


\section{CHAPTER 1 INTRODUCTION}

\subsection{Objective and Scope}

The objective of this study is to investigate the effect of forced vibration on the thermodiffusion measurements in a microgravity environment. This investigation is done by analysing the available experimental data from the IVIDIL $^{1}$ experiment on board ISS $^{2}$. In this study, several parameters such as the temperature and concentration fields in the cavity are studied.

This study is organized as the following: The first and most important stage is to understand the experimental setup and the procedure of the experiment. The mechanism of the apparatus along with the IVIDIL experimental process is discussed in the first stage. The second step is to describe the unique image processing technique. This is done by showing the governing equations and image processing diagram. Lastly, selected experimental runs are analysed not only show the influence of vibration but also the distribution of temperature and concentration in the experimental cavity. This is done for the mixture of (10\% Isopropanol-90\% water) and (50\% Isopropanol-50\% water). The latter goes through further discussion as this is an unstable mixture especially when dealing with high thermal gradient. The mixture of (10\%IPA-90\%Water) is purely aimed to investigate the influence of force vibration on separation ratio. In other words, the impact of Rayleigh vibration on thermodiffusion phenomena is studied in the steady state condition.

The results of this study are indication of valuable information about the conditions of microgravity environment in which future experimental studies could be successfully carried on.

\footnotetext{
${ }^{1}$ Influence of Vibration on Diffusion in Liquids

${ }^{2}$ International Space Station
} 


\subsection{Introduction to Thermodiffusion}

The Swiss scientist Charles Soret, in 1879 , noticed that a salt solution inside a tube which is heated at one end and cooled at the other end would not remain uniform in composition. He showed that under influence of temperature gradient on both sides of the tube, there is a tendency for the mixture to separate [1]. This tendency of mass separation induced by the temperature gradient is called thermodiffusion. Thermodiffusion is a phenomenon observed when temperature gradient is applied to a multicomponent mixture or a solution, which results in molecular segregation and appearance of concentration gradient [2]. Other terms for this phenomenon are Thermal Diffusion, Ludwig - Soret effect, or just Soret effect. In irreversible thermodynamics the cross-effect that assumes the components and heat fluxes between the hot and cold region to be linear function of the concentration difference and the temperature difference [3].

Figure 1-1 shows a 2D model and a graphical representation of an experimental cell that contains a mixture exposed to a temperature gradient.
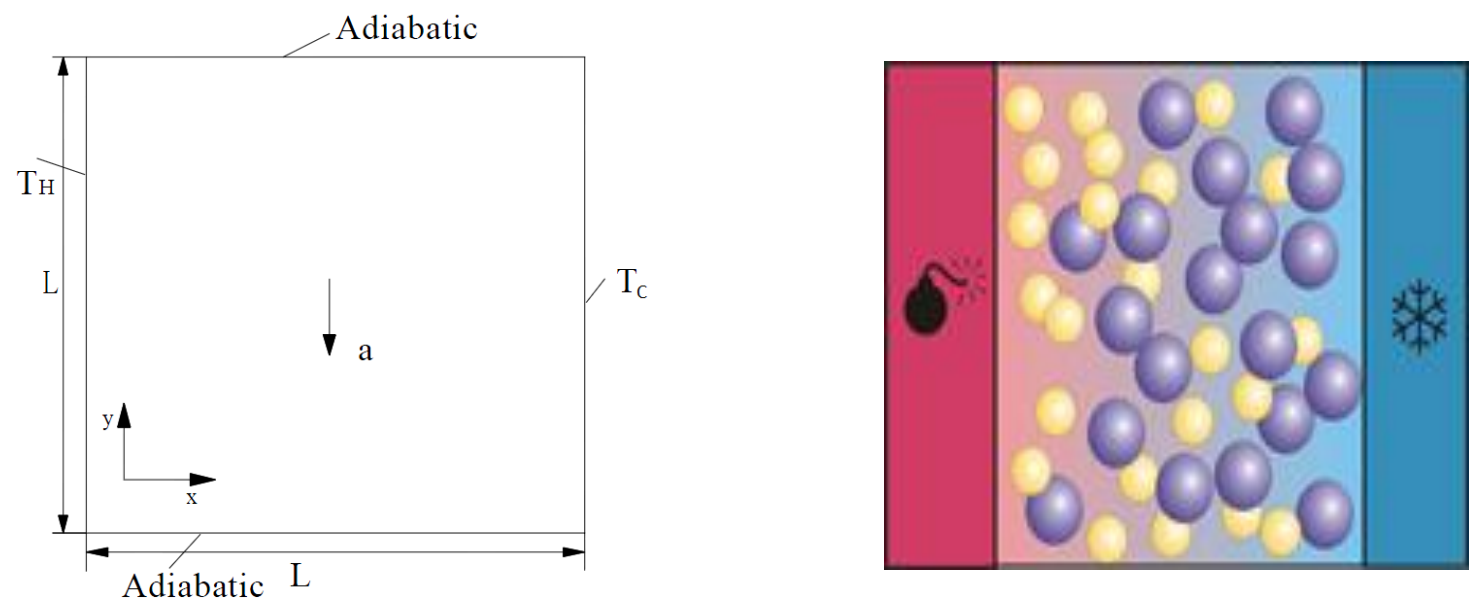

Figure 1-1: 2-D Model of thermodiffusion cell [4]

The thermal gradient is caused by heating the cell from one side and cooling it from the other side. The component of experimental mixture will have the tendency to move to opposite directions along the temperature gradient. Thermodiffusion causes mass flux which acts against diffusion. 
Thermodiffusion causes mass flux which acts against diffusion. There is a challenge between mass flux and diffusion which determines the separation of components of a mixture. Soret coefficient determines the equilibrium condition between the two forces and is the ratio of thermodiffusion coefficient upon molecular coefficient [5]. In general, at the onset of a concentration gradient, a counter measure in the form of the Fickian diffusion process is simultaneously initiated. This Fickian diffusion tries to re-establish the equilibrium [6]. In a binary mixture the mass flux, $J_{m}$ can be written as

$J_{m}=-\rho D \nabla c-\rho\left(1-c_{o}\right) D_{T} \nabla T$

where $\rho$ is the density of the mixture, $c_{0}$ is the concentration of the reference component, $D$ is the Fickian diffusion coefficient and $D_{T}$ is the thermodiffusion coefficient. $\nabla c$ denotes concentration gradient and $\nabla \mathrm{T}$ denotes temperature gradient. The first term in the right hand side of equation 1-1 describes homogenization by normal diffusion, whereas the second term describes mass separation due to a temperature gradient. At steady state condition, i.e. those two terms are of opposite sign and equal intensity and there is no mass flux. This means $J_{m}=0$ and

$\nabla c=-S_{T} c_{o}\left(1-c_{o}\right) \nabla T$

Where

$S_{T}($ Soret coefficient $)=\frac{D_{T}}{D}$

The Soret coefficient characterizes the size of the effect in a binary mixture. Soret coefficient could be positive or negative based on separation of components to the cold or hot region. It has a positive sign when components migrate to the cold side and a negative sign when components migrate to the hot side. Sign changes of the Soret coefficient are well known for a number of small-molecules fluid mixtures. An example of these mixtures is the mixture of water and ethanol, which has been studied by several researchers. The range of Soret coefficient for usual organic mixtures or aqueous solutions is $\left|S_{T}\right| \sim 10^{-3}-$ $10^{-2} \mathrm{~K}^{-1}[1]$. There is also another quantity used to represent thermodiffusion process. This 
quantity is thermal diffusion factor, $\alpha_{\mathrm{T}}$, which is linearly related to the Soret coefficient as

$$
\alpha_{T}=T S_{T}
$$

Thermal diffusion factor is a quantitative measure of the separation. More precisely, the magnitude of $\alpha_{\mathrm{T}}$ indicates the strength of separation of the component with respect to the other species in the mixture. Further, a positive $\alpha_{\mathrm{T}}$ implies that the component segregates to the region with lower temperature. That being said, Pan et al. [7] suggested that accuracy of thermal diffusion factor relies on the accuracy of thermodynamic properties from equation of state, corresponding Fick's diffusion coefficients, and the thermal diffusion modelling. In the literature, the thermodiffusion process is represented quantitatively using one of $\mathrm{D}_{\mathrm{T}}$, $\mathrm{S}_{\mathrm{T}}, \alpha_{\mathrm{T}}$

\subsection{Applications of Thermodiffusion}

Thermodiffusion is considered as a complex physiochemical phenomenon [8] to model, because the heat and mass flux happens simultaneously under non-equilibrium conditions. Thus, there is no adequate understanding of the theory of thermodiffusion. However, for the past century, there have been extensive researches both experimental and theoretical dedicated to investigate this phenomenon. Thermodiffusion or Soret effect has been undergoing considerable amount of research and study in various systems such as gases, electrolytes, liquid hydrocarbons, alcohols, ferrofluids, polymers, proteins, combustion, surfactant micelles, latex particles, etc. [6]. Most of the studies have been dedicated to the petroleum industry. These industries conduct vast amount of research to understand thermodiffusion and determine the fluid distribution in reservoir [9]. Thus, understanding thermodiffusion is of great importance to petroleum industry. This is because in the underground oil reservoirs the geothermal gradient coupled with the nearly motionless conditions initiates this process, thereby determining the distribution in the reservoir. Generally, there are various forces contributing to distribution of fluid such as gravity, thermal gradient and other fluxes. It is an important challenge to take all these phenomena 
in to account to measure the connectivity of the different panels and layers in an oil field [10]. Touzet et al. [2] believe that the reverse geothermal gradient, through thermodiffusion and the gravity field, through gravitational segregation, are the two phenomena that model the composition of the fluid in petroleum reservoir. Furthermore, thermodiffusion has been employed to understand convection in stars [11], demographics of an ocean [12], and isotropic separation of liquids. Also, study of thermodiffusion plays significant role in emerging applications such as particle manipulation by temperature gradient for microfluidic applications, and in optical screening methods for biomolecules and colloids [13].

\subsection{Experimental Measurements of Thermodiffusion}

Due to the smallness of the thermodiffusion coefficient and the possibility of its coupling with other methods of mass transfer such as convection, accurate and reliable measurements are difficult to perform under practical conditions. There are several experimental techniques to measure and determine Soret effect and factors governing this phenomenon for the past century. The techniques have been divided in to two main groups by some researchers and scientists. First group of experiments are classical in terms of their use and functionality. These include the classical Soret cell, thermogravitational column, and the two chamber thermodiffusion cell. Second group involves optical method such as laser beam deflection technique, thermal diffusion forced Rayleigh scattering, and the thermal lens technique. Optical methods have been utilized to study the thermodiffusion process in liquid mixtures, polymer solutions and colloidal suspensions.

All the above experimental approaches have their advantages and disadvantages. There is no exact way of distinguishing or categorizing these technical approaches. Some of the classical approaches have been used longer and more often because of their accuracy and ease of use. Other techniques have been developed recently and bring more advantages to the table. To avoid tedious discussion, only two of each technique is explained in this section for the purpose of comparison. 


\subsubsection{Classical Approaches}

The Classical methods have advantages of being fairly straight forward and the construction of the equipment is simple. It is also less expensive to set up classical apparatus to measure thermodiffusion. Instruments that are used in this group tend to achieve larger separation ratio. Thus, more accurate results are obtained. This group of technique uses convective coupling to obtain better separation. On the other hand, the drawback of these techniques is the inevitable mechanical disturbance to the system that introduces convection in the mixture that can destroy the Soret separation.

\subsubsection{Classical Soret Cell}

A standard Soret cell is constructed with two horizontal rigid plane plates. These plates are typically made of stainless steel or copper for their heat conduction capabilities. Figure 1-2 shows the schematics of such apparatus.

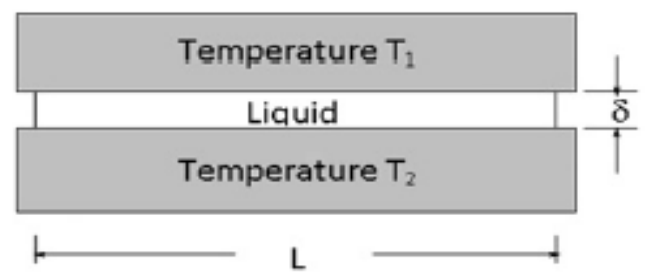

Figure 1-2: Schematic of a classical Soret cell [6]

There is a thin gap of thickness $\delta$ between the plates and it is maintained by brace. The experimental fluid mixtures are kept in this gap of usually $2 \mathrm{~mm}$ and induced by a heated top and a cooled bottom plate [14]. The reason to apply thermal gradient from the top plate is to avoid free convection [6]. The brace that keeps the plates steady is made of material that has very low thermal conductivity such as PVC [15]. There are holes drilled on the brace in order to draw samples. Number of holes is at least one, but several of them are usually drilled to follow the kinetics of the separation [1]. There is a watertight device in each hole where samples are drawn by needle. Each time, small portions of samples (i.e. $0.2 \times 10^{-3} \mathrm{~cm}^{3}$ ) are drawn closed to the isothermal horizontal boundaries through curved hypodermic needles [15]. Samples are analysed with high-resolution refractometer 
$(\mathrm{n} \pm 0.00001)$ or densimeter $\left(\rho \pm 0.000001 \mathrm{gr} / \mathrm{cm}^{3}\right)$. The density or the refractive index of the experimental sample is determined to get the concentration difference, $\Delta \mathrm{C}$, close to the two plates. The result is compared with calibration curves [1]. Possible non-homogeneities are taken in to account by measuring $\Delta \mathrm{T}$, the temperature difference, at each sampling position [15]. Both the concentration and temperature differences are used to calculate $S_{T}$ using equation 1-5, which illustrates linear relationship at steady state.

$$
\Delta C=-S_{T} c_{o}\left(1-c_{o}\right) \Delta T
$$

In contrast, some researchers use local value of temperature at each location compare to the temperature difference between the two bounding plates. Costeseque et al. illustrated this method using cells with six holes and removing samples at different times to follow the time evolution of $\Delta \mathrm{C} / \Delta \mathrm{T}$ [15]. Following equation illustrates the concentration difference as a function of regular time intervals.

$$
\frac{\Delta C(t)}{\Delta T}=-S_{T} c_{o}\left(1-c_{o}\right)\left(1-\frac{8}{\pi^{2}} \sum_{\text {odd } n} \frac{e^{-n^{2} t / \tau}}{n^{2}}\right)
$$

Where the relaxation time, $\tau$, is related to diffusion coefficient as shown in equation 1-7:

$$
\tau=\frac{\delta^{2}}{\left(\pi^{2} D\right)}
$$

Through equation1-5 and 1-6 $\mathrm{S}_{\mathrm{T}}$, and D are determined. Thus, the thermodiffusion coefficient, $\mathrm{D}_{\mathrm{T}}$, is obtained. Wiegand in his study mentioned that equilibration time for low molecular mixtures is approximately $\tau=10 \mathrm{~min}$, and for polymeric systems is around 10 hours and more. Consequently, a more adequate temperature control and stability of the cells are required when equilibration times are longer.

The classical Soret cell apparatus is straightforward in terms of simplicity of equipment and operational procedure. Versatility is a further inherent advantage in Soret cell [16]. In contrast, there are some unavoidable disadvantages in this experimental method. First, this approach is subjected to convection due to small sampling procedure that disturbs the 
experimental system and influence the precision of the concentration gradient induced by the temperature gradient. One can suggests making the top and bottom plate thinner in the cell to slightly avoid convection. Longree et al. use thin-walled stainless steel plates, in order to protect the system against convection [17]. Second, this approach requires long equilibration time especially for polymer solutions. This results from using porous medium to mitigate the convection effects [6]. Third, unavoidable temperature gradient in this apparatus is another drawback. This is because plates need to be maintained at constant temperature for mixtures with long equilibration time. Wiegand suggested using larger plates hanging over the part containing the sample [14]. This would reduce the temperature gradient end effects at the edge of the plates. These drawbacks could be slightly neglected if flow cells are used instead of static cells. This method is described by Butler and Turner in studying Soret effect in aqueous solution of $\mathrm{CdSO}_{4}$ at mean temperature of $25^{\circ} \mathrm{C}$ [16]. They start their study by making comparison between classical cells and flow cells. The earlier cells suffered from small leaks and performed moderately at the lowest flowrate. [18]. Hence, they suggest reducing the duct depth to increase the flowrate. Also, the flowcell can provide large samples which can be analysed at convenient. Furthermore, Soret cell experimental approach lacks accuracy in investigating mixtures with negative $\mathrm{S}_{\mathrm{T}}$. BouAli et al. [18] suggested maintaining the Grashof numbers greater than a certain critical value in order to precisely measure negative Soret coefficient.

\subsubsection{Thermogravitational Column (TGC) Technique}

Thermogravitational column consists of two vertical plates maintained at two different temperatures that would create horizontal temperature gradient. Figure 1-3 shows schematic of a thermogravitational column.

The typical height of column is about $40-50 \mathrm{~cm}$ and the gap between the plates is

maintained between 1 and $2 \mathrm{~mm}$ [6]. Two spacers $C$ and $D$ enforce a fix space between the 


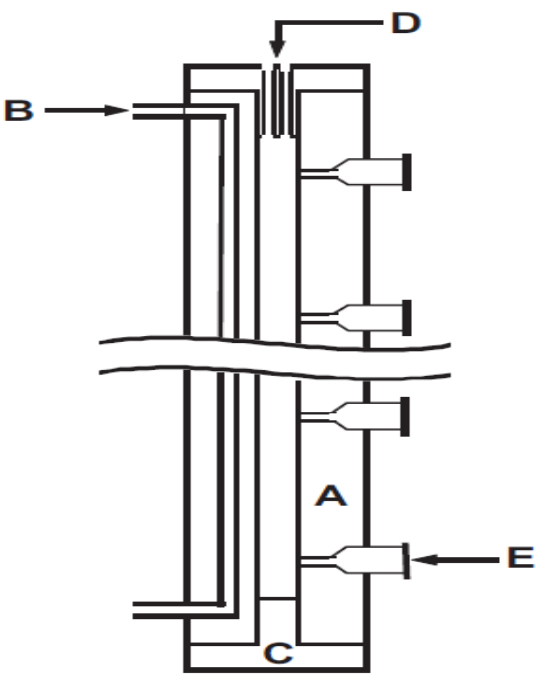

Figure 1-3: Schematic of thermogravitational column [10]

two walls. The upper spacer consists of two small holes for filling of the column and for air to escape during the filling [1]. The temperature gradient is introduced by maintaining one wall at a higher temperature. Consequently, Soret effect causes each component move to different sides resulting lateral density gradients (i.e. component that separates toward the cold side goes to the bottom and the other component goes to top). This would produce buoyancy driven convection roll in the annular region [6]. Small samples (1 to $2 \mathrm{~mL})$ are drawn from sampling ports $\mathrm{E}$ and analysed for their composition. Numbers of ports are usually five or six along the column height.

There are two different approaches to determine $S_{\mathrm{T}}$ in TGC method. The first approach involves two steps to find $\mathrm{D}_{\mathrm{T}}$ and $\mathrm{D}$. Initially, drawn samples are analysed at steady state to obtain the concentration distribution along the column height to find $\mathrm{D}_{\mathrm{T}}$. Then $\mathrm{D}$ is determined using a different experiment in an open ended tube. Second approach involves recording the time history of the velocity amplitudes in the mixture using laser Doppler velocimetry [6].

In the first approach, mass fraction difference between top and bottom of the column is determined, which in turn translates to the thermodiffusion coefficient via the Furry-JonesOnsager theory [6]. Equation 1-8 illustrates the theory behind finding $\mathrm{D}_{\mathrm{T}}$ from concentration distribution along column. 
$c=504 \frac{v}{\alpha g} c_{o}\left(1-c_{o}\right) \frac{h}{\delta^{4}} D_{T}$

$\alpha$ is the thermal expansion coefficient of the mixture, $g$ is the acceleration due to gravity, $v$ is the kinematic viscosity, $\delta$ is the gap width between the walls in the $\mathrm{x}$-direction and $\mathrm{h}$ is the height of the column.

The alternative or indirect way to quantify the Soret effect is based on the modification of the velocity field. Dutrieux et al. [19] explain the theoretical and experimental details of this approach. They mention that in steady state, the horizontal gradient will not affect the buoyancy force and increase in velocity amplitude is transitory in time [1]. The amplitudes are recorded using laser Doppler velocimetry. Generally, results will show initial increase of velocity from zero to $V_{\max } \mathrm{m} / \mathrm{s}$ and then eventually decrease to steady state $\mathrm{V}_{\text {steady }} \mathrm{m} / \mathrm{s}$ [6]. The relative difference between the maximum velocity and the steady state value is equal to the separation ratio and related to Soret coefficient. Equation 1-9 shows the relationship between separation ratio of the mixture and $S_{\mathrm{T}}$.

$\phi=\frac{\beta}{\alpha} S_{T} c_{o}\left(1-c_{o}\right)=\frac{V_{\max }}{V_{\text {steady }}}-1$

TGC method has been utilized by many scientists to investigate thermodiffusion in gases as well as liquid mixtures. Also, investigations have been made for hydrocarbons, associating mixtures, polymers, and Ferrofluids by this method [6]. Moreover, researchers have been using TGC method to study behaviour of not only binary mixtures, but also ternary and multicomponent mixtures under Soret effect. This approach was first recommended by Clusius and Dickel in late 1930's [6]. However, TGC method has gone through many modifications in terms of design and operation. There are various versions of TGC apparatus in literature, which are just named here to avoid tedious details. Some of these types include concentric cylindrical wall TGC, low-temperature thermodiffusion column made of glass, parallel plate version, and high pressure TGC [6]. Hence, TGC method has a large applicability and varieties in its apparatus. Besides, Thermogravitational column uses convection to enhance the separation ratio of the components. This would add 
to its advantages, since convection is an interruption in studying purely diffusive phenomena. Also, one could use the inclined TGC apparatus to create larger separation in the column that positively influences precision of this method. Platten et al. recommend that molecular separation between the top and the bottom in a thermogravitational column can be substantially increased by inclining the column by an angle $\theta$. This increase is given by $1 / \cos \theta$ [20]. This would result in a better accuracy on $\mathrm{D}_{\mathrm{T}}$. Moreover, TGC can be employed for mixtures with negative $S_{\mathrm{T}}[18]$. Bou-Ali et al. study the stability of a situation when horizontal temperature gradient is applied across a vertical layer of a binary fluid with negative Soret coefficient [18]. Their conclusion shows that measuring negative Soret coefficient is possible considering a large gap width between the plates and sufficiently large temperature difference in thermogravitational column. This would maintain the Garshof number larger than a certain critical value.

On the other hand, small gap between the walls $(1-2 \mathrm{~mm})$ is a drawback in this experimental approach. Also, marginal movement or angling the apparatus would increase or decrease the separation and affect the value of Soret coefficient. Moreover, there are some simplifications in deriving governing equations to calculate concentration gradient and $\mathrm{D}_{\mathrm{T}}$. Consequently, experimental errors occur as a result of those simplifications.

\subsubsection{Optical Techniques}

Optical techniques have been used for a considerable period by researchers. Optical methods have high sensitivity and can be used to investigate even dilute solutions. The most significant advantage of these methods is that convection can be greatly avoided and the measurements can be made without disturbing the system. In other words, convection effect could be neglected because these methods tend to not disturb the transport process [14]. Moreover, these techniques involve very precise and complex setup requiring delicate laser adjustments. 


\subsubsection{Soret Cell With Beam Deflection (BD) Technique}

This method is one of the optical approaches to measure the Soret effect. The apparatus is similar to the Soret cell approach with some modifications. However, Soret coefficient is measured in a completely different way than in Soret cell method. The main modification in BD technique is that two opposite lateral walls are replaced by glass of good optical quality. The reason to use glass wall is to easily obtain the refractive index of experimental mixture through deflection of beam. In the absence of any thermal or concentration gradient, a laser beam perpendicular to one of the glass walls would pass through the mixture in a horizontal path without any deflection. The gradients would cause the laser beam that is travelling in an index of refraction field to not propagate horizontally [1]. The schematic of this apparatus is shown in Figure 1-4 along with initial and steady state points of incident. Point A illustrates the point that laser beam initially crosses the mixture without any reflection and hits the beam detection screen. On the other hand, point B represents the steady state condition when reflection is done due to presence of gradients (thermal or concentration). The distance between these two points of incident on the beam detection screen shows the variation of the refractive index.

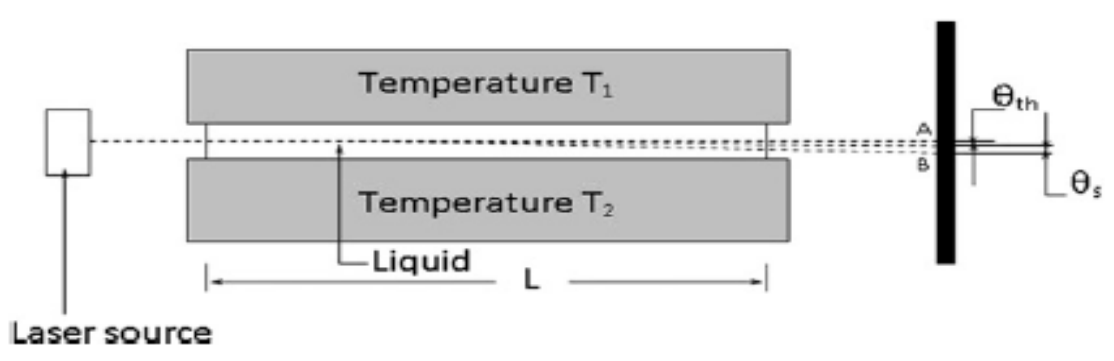

Figure 1-4: Schematic of beam deflection apparatus. A and B are the initial and steady state points of incidence of laser beam on the screen [6]

The relationship between the refractive index and gradients is shown below.

$$
\nabla \eta=\frac{\partial \eta}{\partial \mathrm{T}} \nabla \mathrm{c}+\frac{\partial \eta}{\partial \mathrm{T}} \nabla \mathrm{T}
$$


$\partial \eta / \partial \mathrm{T}$ and $\partial \eta / \partial \mathrm{c}$ are obtained from two calibration curves [1]. The relaxation time for establishment of temperature gradients varies with of the concentration gradient. In general, there are two orders of magnitude difference between both relaxation times since the diffusion coefficient is smaller than the thermal diffusivity in liquids [1]. This means that there are two different deflections corresponding to each gradient. According to Saghir and Srinivasan [6], the relaxation time for establishing thermal gradient is much smaller than the concentration gradient. The initial deflection, $\Delta \theta_{\text {th }}$ occurs by applying thermal gradients. Subsequently, as the concentration gradient is built up in the mixture, the laser beam undergoes another deflection that is $\Delta \theta_{\mathrm{s}}$. Equation 1-11 shows how Soret coefficient is obtained using the two deflections and gradient of the refractive index.

$S_{T}=-\frac{1}{c} \frac{\partial \eta / \partial \mathrm{T}}{\partial \eta / \partial \mathrm{c}} \frac{\Delta \theta \mathrm{s}}{\Delta \theta \mathrm{th}}$

Platten explains that diffusion relaxation time and corresponding diffusion coefficient can be determined if one deviation follows the kinetics of the second deviation of the beam [1]. Alternatively, providing uniform temperature between the two plates, the diffusion coefficient can also be obtained after reaching steady state [1]. The temperature gradient can be switched off and the two glass walls will quickly return to zero temperature. Temperature gradient is destroyed faster, but the concentration gradient will take longer time to disappear. As the concentration gradient reduces to zero, the diffusion coefficient can be determined following the time dependence of the deflection angle that needs to return to the original horizontal path.

The BD approach has been used by researchers and scientist to measure Soret effect for the past century [6]. Great percentage of investigations has been done on binary mixtures, especially the water/ethanol system. This mixture has widely been studies to understand the double diffusive convection in binary mixtures [21]. Some researchers have tried to explore the postulates of second sign change when the concentration of ethanol is high [22]. Recently, BD approach has been developed to a micro-scale version, which is used by Putnam and Cahill [23]. They did their experiments on $26 \mathrm{~nm}$ polystyrene colloids with 
carboxyl functionality $(\mathrm{COOH})$ in water. They suggested that micro-scale geometry of beam deflection method speeds up equilibration by a factor of approximately 300 [23]. The temperature gradient is produced by heating of $250 \mathrm{~nm}$ thick gold wires using a square wave voltage signal. The wires are patterned by photolithography and chemically etched. In this apparatus, the optical path is very small and detection is obtained in the frequency domain via a lock-in amplifier.

The advantages of beam deflection (BD) technique come from simplicity of design of the apparatus itself and its operating principles. The path length of the beam in the cell is 1$4 \mathrm{~cm}$ and the approximate spacing between two plates is 1-2 $\mathrm{mm}$ [6]. Beam deflection approach has been employed to investigate Soret effect in binary systems as well as ternary mixtures. Another important advantage of this technique is accuracy of the measurements, which are made without physically disturbing the system. In contrast, the main disadvantage of $\mathrm{BD}$ method is the equilibration time that varies depending on the working mixtures. Incident of laser beam during a long equilibration time would lead to heating the liquid mixture. In turns, undesirable effects could be caused by extra heating. Also, longer the equilibration time, higher precision and control are required. Saghir and Srinivasan [6] suggest narrowing the interpolate gap to somehow reduce the equilibration time. They also mention the limitation of this solution since the laser beam cannot be focused within the cell to a small spot.

Moreover, Putnam and Cahill [23] eliminate the above problems and reduce the equilibration time by using their micro-scale BD apparatus.

\subsubsection{Thermal Diffusion Forced Rayleigh Scattering (TDFRS)}

The forced Rayleigh light scattering provides a useful and sensitive tool for the investigation of thermodiffusion. This method is to study thermal diffusive behaviour of mixtures with holographic grading technique. The measurement principle is the following: a laser beam is split into two beams of equal density. These two beams are converged at the experimental sample. The interference of these laser beams produces an intensity grading in 
the liquid mixtures [14]. A small amount of dye, dissolved in the sample, converts the intensity grading into a temperature grading, which in turn causes a concentration grading by the effect of thermodiffusion [24]. The purpose of dissolving dye in the mixture is to ensure sufficient absorption of the wavelength of the writing beam. The induced temperature and concentration gradients result in a refractive index gradient in the mixture, which is read out by the diffraction of a third laser beam [6]. The resulting laser beam is related to the Soret coefficient as in equation 1-12:

$\zeta=1-\left(\frac{\partial \eta}{\partial c}\right)\left(\frac{\partial \eta}{\partial T}\right)^{-1} S_{T} c(1-c)\left(1-e^{-q^{2} D t}\right)$

Where $\mathrm{q}$ is the grading vector and $\zeta$ is the heterodyne signal intensity. $\left(\frac{\partial \eta}{\partial c}\right)$ and $\left(\frac{\partial \eta}{\partial T}\right)$ are contrast factors, which are calibrated values obtained separately. Figure 1-5 shows a schematic of the TDFRS setup.

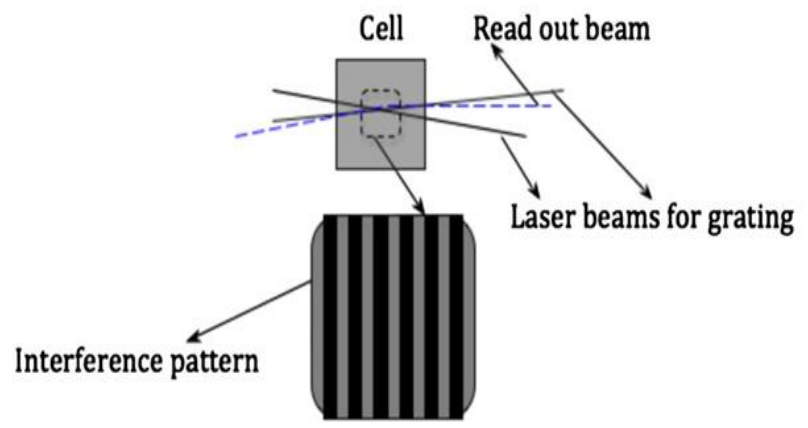

Figure 1-5: Schematic of thermal diffusion forced Rayleigh scattering setup [24]

TDFRS has been employed to study Soret effect in liquid mixtures of hydrocarbons, polymers, biopolymers, ferrofluids, and colloids aqueous as well as non-aqueous solutions. The advantage of this method is that absolute intensity measurements and calibration with a known standard are not required [25]. Also, very low temperature variations can be realized, which adds to benefits of TDFRS approach. The concentration gradients assure that the system is close to equilibrium. This lead to equilibration times of the order of a few hundred milliseconds that enables measurement of small diffusion coefficients [6]. 
Ning et al. studied the effect of dye addition on thermodiffusion measurement [26]. As it was mentioned, dye is used to increase light absorption and creation of temperature grating. The resulting temperature gradient can have negative implication and influence the thermodiffusion process. Hence, inaccurate results would be obtained from experiments. As a result, some researchers suggested avoiding the use of dye for aqueous systems. Furthermore, TDFRS involves a complex and expensive setup requiring delicate laser adjustments. Also, this method does not provide accurate results when the index of refraction is at the maximum, for mixtures such as water-alcohol (90\% or higher) [6].

Nevertheless, investigation of Thermodiffusion phenomena through those experiments could be even more precise providing a microgravity environment. This kind of condition is available on space platforms such as free fly satellites, FOTON M3, and the International Space Station (ISS). There are specific advantages and disadvantages concerning thermodiffusion experiments in space, which are addressed in the next section.

\subsection{Thermodiffusion of Binary Fluid Mixture in Microgravity}

There is no universal technique that works for measuring the Soret coefficient of any binary mixture. Each technique has its own limitations. Besides, all experimental techniques require establishing temperature gradient in the fluid in gravity field. This condition easily provokes convection in terrestrial conditions which disturbs or even erases component separation. The effect of convection is more pronounced in the case of negative Soret when the heavy liquid goes to the hot wall [27]. The buoyancy force has significant effects on behaviour of the mixture when the temperature gradient is applied perpendicular to the gravity vector. A numerical simulation is performed for the benchmark case. A 3-D cubic domain is chosen which is filled with the mixture of water $90 \%$ and Isopropanol $10 \%$ and is subjected to different gravity fields. The direction of applied gravitational acceleration is perpendicular to the thermal gradient. Various combination of gravity fields are examined in this study. For case of residual gravity field, different values of static gravity field orthogonal to the temperature gradient are simulated. 
The results are included in Figure 1-6 in which the separation of species has been normalized in accordance to the ideal condition. This figure illustrates the effect of different levels of gravity on the intensity and duration of the component separation. In other words, it demonstrates the negative effect of convection inside the cell on the separation of the species. According to the same figure, starting from $\mathrm{g}=10^{-4} \mathrm{~g}_{\mathrm{o}}$, a local maximum appears at $\mathrm{S}_{\mathrm{R}}$ profile and as time passes, it goes towards steady value. This means that for gravity fields with residual value of larger than $10^{-4} \mathrm{~g}_{\mathrm{o}}$ the negative effect of gravity on the thermodiffusion experiment is significant.

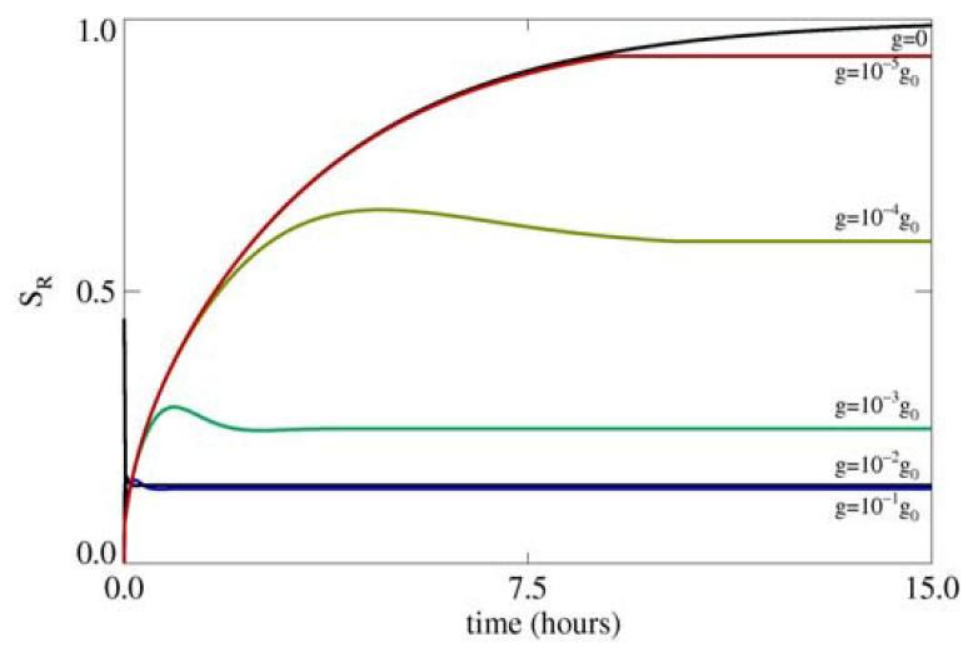

Figure 1-6: Transient Soret separation, $S_{R}$ at different static gravity levels, $\nabla T \perp g$ [28]

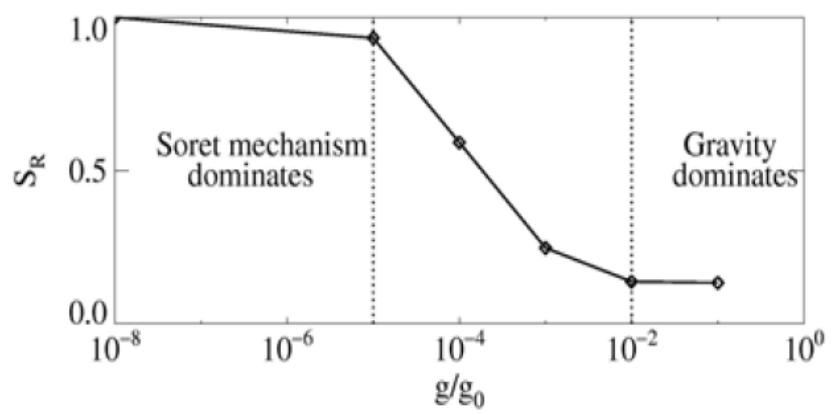

Figure 1-7: Dependence of the steady state Soret separation on the gravity level [28] 
The steady values of $S_{R}$ may be presented as a function of gravity level. Figure 1-7 shows the different mechanisms in mass transport.

Diffusive mechanisms dominate in the component separation at low gravity (i.e. at $\mathrm{g} \leq$ $10^{-5} \mathrm{~g}_{\mathrm{o}}$ ). The slope of graph in the range $10^{-5} \mathrm{~g}_{\mathrm{o}}<\mathrm{g}<10^{-2} \mathrm{~g}_{\mathrm{o}}$ indicates the importance of both the diffusive and convective mechanisms in mass transport. The gravitational effects start to dominate at the end of the middle range. Thus, buoyancy force controls the mixing after $\mathrm{g}>10^{-2} \mathrm{~g}_{\mathrm{o}}$. The perturbation effects increase with the Rayleigh number in thermodiffusion cells used for non-electrolytic solutions. The cconvection may alter the gradient of concentration by several orders of magnitudes [29]. Note that convection can also appear in theoretically stable configurations due to the non-ideally of an experimental setup, which lead to unstable temperature (density) stratification [30].

\subsubsection{Microgravity Experiment}

Measurements conducted in controlled microgravity environment minimize the perturbation effects of gravity and allows the true diffusion limit to be achieved. The microgravity environment provides unique tool for studying the behaviour of liquids and measurements of transport coefficients [30]. Thermodiffusion experiments have been performed in microgravity environment benefiting from the reduced gravity condition. Thus, it is beneficial to grow knowledge of microgravity environment and its characteristics.

Microgravity environment is defined as the acceleration conditions that exist on a microgravity platforms and orbital facilities such as sounding rocket, drop tower etc., where the gravitational force is balanced by the inertia force. The platform either can be an orbital facility such as ISS or an airplane in parabolic flight. In practice a pure zero-gravity field is impossible and there is always a residual value for the acceleration level. For a microgravity environment, the residual gravity is many orders of magnitude smaller than 
the sea level gravitational acceleration value known as $g_{0}$. Typically, the residual gravity acceleration for platforms orbiting in Low Earth Orbit (LEO) is in order of $10^{-6} g_{0}$ [29]. The behaviour of fluids in a microgravity environment motivates material science and life science experimentation. Several phenomena may have direct or indirect influence on the fluid behaviour, which could be exploited to improve a number of space processes [29].

The absence of hydrostatic pressure gradient in these environments may result in different phenomena including formation of large droplets and liquid bridges [31]. Moreover, as a result of elimination of buoyancy force the processes are governed by diffusion rather than convection. The former consequence can be utilized for performing thermodiffusion experiments.

\subsubsection{Microgravity Carriers}

The microgravity environment is created in free falling carriers, where the sum of all forces, other than gravity, acting on the carriers is significantly reduced. Scientific and technological experimentation in microgravity can be conducted in different carriers on Earth or in orbit. However, providing a weightless environment on Earth imposes practical constrains on the level and duration of the microgravity required for investigations and on the cost at which this environment is obtained. Nevertheless, technology improvements and availability of platforms such as the International Space Station (ISS), airplane in parabolic flight, drop tower and sounding rockets, provide a unique opportunity to demonstrate experiments in microgravity environment in space. Here are some of the microgravity carriers for the purpose of comparison.

\subsubsection{Drop Tower, Drop Tubes, Sounding Rockets}

In these platforms gravity of earth is neutralized in an accelerating vertical motion in which can either be an upward decreasing speed or downward increasing speed. For case of sounding rockets, rockets are used to carry instruments from 50 to 1,500 kilometres above 
earth surface. The average flight time is less than 30 minutes, usually between 5 and 20 minutes [32]. The rocket consumes its fuel on the first stage of the rising part of the flight, then separates and falls away, leaving the payload to complete an arc and return to the ground under a parachute. The main advantage of using sounding rockets is their considerable longer microgravity duration ( 15 minutes). Also, sounding rockets are relatively inexpensive compared to other large orbital satellites operating scientific experiments.

\subsubsection{Airplane in Parabolic Flight}

In these platforms an aircraft is used to provide the sensation of weightlessness by following an (approximately parabolic) elliptic flight path relative to the centre of the Earth. While following this path, the aircraft is in free fall at certain points of its flight path. A typical trajectory of zero-g airplane is shown in Figure 1-8. Generally, this method can provide up to 30 seconds of microgravity at a cost in the range of a few dollars per pound. Aircraft parabolic flights are useful tools for performing short duration scientific and technological experiments in reduced gravity. There are several advantages in performing experiments in these kinds of environments. The advantages are the short turn-around time, the low cost of experiment, the flexibility of experimental approach, the possibility of direct intervention by investigators on board the aircraft during and between parabolas, and the possibility of modifying the experiment set-up between flights [33]. Aircraft parabolic flights attain reduced $g$ levels, ranging $10^{-2}-10^{-3} g$. Additional advantage of this experiment is allowing human intervention during experiment in reduced gravity. 


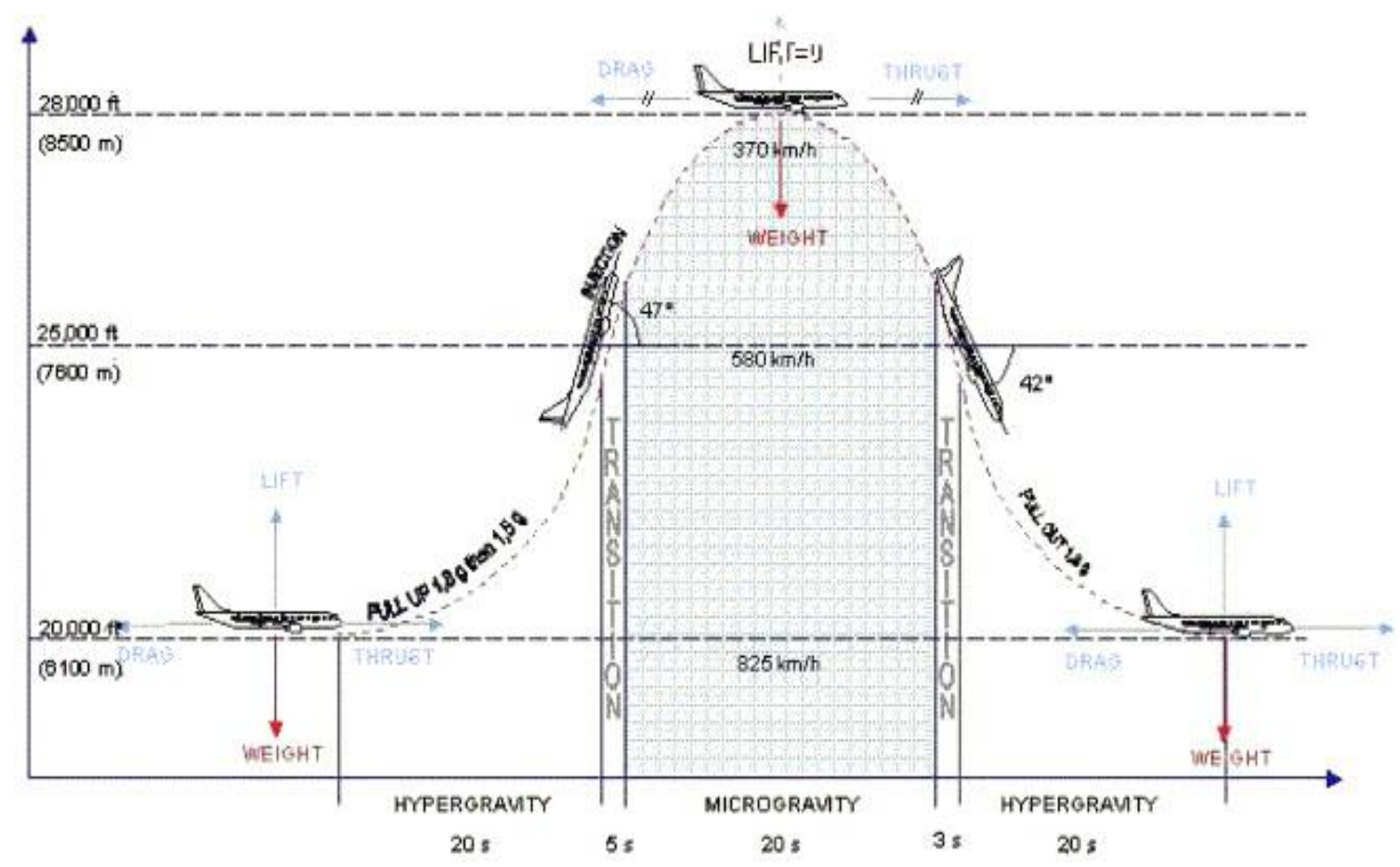

Figure 1-8: Schematic view of the trajectory of parabolic flights [33]

\subsubsection{Point platforms}

This terms is commonly refers to spacecraft and orbiters that are moving in a circular orbit in which the gravity force is balanced by the centrifugal force. There have been numerous unmanned scientific satellites. FOTON-M, for instance, is series of robotic spacecraft used by Russia and the European Space Agency for research conducted in the microgravity environment of Earth orbit [34].

Beside scientific satellites, International Space Station has provided promising platform for microgravity investigations with human supervision. Performing science experiments has always been a part of space platforms. Previously science experiments used to conduct on MIR Russian space station and space shuttles. Nevertheless, none of those platforms were suitable as ISS, essentially because of the available facilities on-board. At this moment, ISS serves as a microgravity and space environment research laboratory in which crew members conduct experiments in biology, human biology, physics, astronomy, 
meteorology and other fields [35]. ISS orbits the earth in a polar orbit (between 388 and $401 \mathrm{~km}$ from the surface of the earth), with an average orbital speed of 7,706.6 m/s.

Comparison between the above microgravity carriers is summarized in Table 1-1 with respect to average characteristics such as microgravity levels and duration, working volume units for experiment set-up, direct or indirect human interaction and preparation time.

\begin{tabular}{llllll}
\hline & $\begin{array}{l}\mu-\mathrm{g} \text { level } \\
(\mathrm{g})\end{array}$ & $\begin{array}{l}\text { Duration } \\
\text { time }\end{array}$ & $\begin{array}{l}\text { Volume } \\
\left(\mathrm{m}^{3}\right)\end{array}$ & $\begin{array}{l}\text { Human } \\
\text { intervention }\end{array}$ & $\begin{array}{l}\text { Prep. } \\
\text { time }\end{array}$ \\
\hline Drop towers and tubes & $10^{-3}-10^{-6}$ & $<5 \mathrm{~s}$ & $<1$ & Indirect & Weeks \\
Aircraft parabolic flights & $10^{-2}-10^{-3}$ & $20-25 \mathrm{~s}$ & $>10$ & Direct & Months \\
Sounding rockets & $10^{-4}-10^{-5}$ & $5-13$ min & $<1$ & Indirect & 1 year \\
Manned orbital platforms & $10^{-2}-10^{-5}$ & weeks-years & $>1$ & Direct & Years \\
Automatic orbital platforms & $\approx 10^{-5 \mathrm{a}}$ & weeks-months & $>1$ & Indirect & Years \\
\hline
\end{tabular}

Table 1-1: characteristics of microgravity carriers [33]

\subsubsection{Disturbance in zero-g conditions}

In all actual microgravity environments, there will be non-zero resultant accelerations that basically disturb the ideal zero-g conditions. Generally the non-zero difference can be separated into steady (or quasi-steady) part $\left(g_{R}\right)$ that is known as residual-g and a time dependent part $g(t)$ that is known as g-jitter. With respect to the orbiting platforms, the disturbances can be due to two types of sources; the "External forces" which act on the platform and that change its momentum (aerodynamic drag, radiation pressure, solar wind, meteorite impacts, docking and orbital manoeuvres by propulsive devices. And "Internal forces" that cause gravity gradient including mass dislocation inside the platform (e.g. crew activity, motion of mechanical parts inside facilities).

Monti [29] identifies the International Space Station disturbances in the following categories: 


\subsubsection{Steady (or quasi-steady) Residual-g}

These include aerodynamic drag $\left(1 \sim 3 \times 10^{-7} g_{0}\right)$, radiation pressure $10^{-8} g_{0}$ (that is periodic with the orbit). For points distant from the centre of mass, gravity gradient and centrifugal forces due to the ISS rotation exist.

\subsubsection{Pulses-like (single or compensating) Accelerations}

The main sources of these accelerations are the thruster firing $\left(10^{-4} g_{0}\right)$, crew activities $\left(10^{-3} \sim 10^{-2} g_{0}\right)$ or external forces (docking/berthing, of the order of $\left(10^{-4} g_{0}\right)$, micrometeorite impacts $\left(10^{-9} g_{0}\right)$.

\subsubsection{Periodic High Frequency Acceleration}

This category is the most concern of fluid experiments and they are due to on board machineries and natural frequencies excited by external or internal actions $\left(10^{-6}<g / g_{0}<10^{-2} ; 0.1 \mathrm{~Hz}<f<300 \mathrm{~Hz}\right)$.

The former two accelerations are known as "g-jitter" and are essentially random highfrequency vibrations. These vibrations are measured by some probes mounted on the space station. The residual gravity is rather constant and does not change significantly over time. The predicted value of $g_{R}$ (for the ISS) is of the order of magnitude of $0.5 \mu \mathrm{g}$, for the US Lab, between 1 and 1.8 $\mu$ g, for European COF, and about $2 \mu \mathrm{g}$ for Japanese module (JEM). The schematic picture of ISS is illustrated in Figure 1-9 indicating the residual acceleration for different modules. The methodology for collecting vibration data on board ISS will be discussed in Chapter 2. 

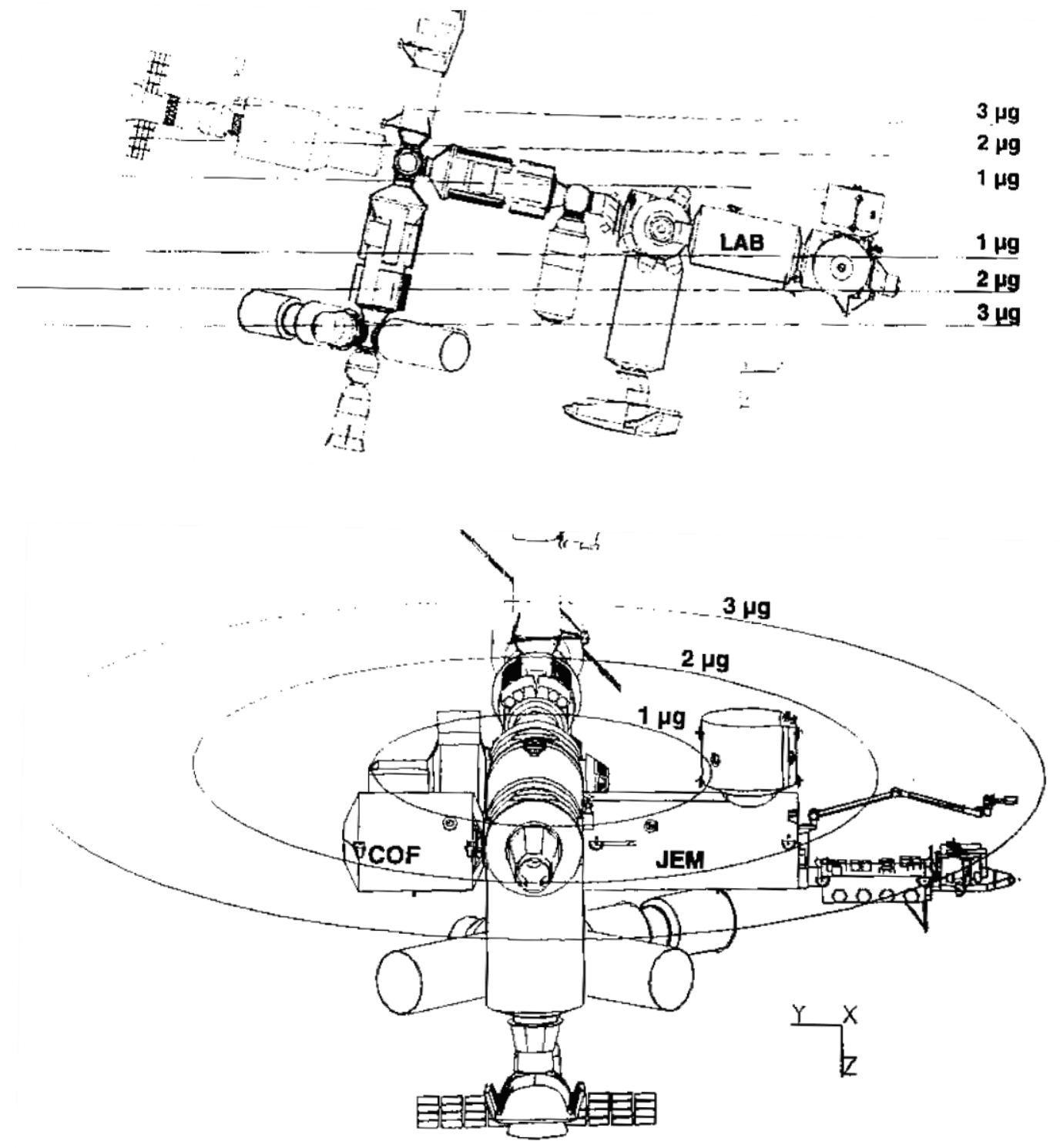

Figure 1-9: Approximate residual-g on the ISS [29]

As mentioned above, availability of microgravity environment provides a unique environment for fluid experiments. A few of such experiments is devoted to thermodiffusion measurements. FOTON satellites (launch 2002 - 2007) where used for thermodiffusion experiments [36]. On ISS, Columbus laboratory has been the host of various fluid flow experiments since its activation in 2008 [37]. Some of them dedicated to measurements of multi-component liquid mixtures and metallic melts that are impossible to determine with a comparable precision on the ground. However, there have been a great 
number of discussions about the effects of micro-accelerations that might affect the ideal sense of the results.

Consequently, results that are obtained from ISS experiment could be used as benchmarks since there is a significant reduction in the convection as a result of reduced gravity and minimized disturbances. In addition, microgravity environment can provide good insight in studying Soret effect especially for mixtures with low viscosity because of their sensitivity to gravity induced instabilities [6]. However, some might argue whether running thermodiffusion experiment in space is absolutely necessary. The drawbacks of such experiment bring up debates about providing microgravity environment. There is high cost involved in performing an experiment in space. This would reflect on number of experiments that are feasible in a single mission. Also, there are some constrains including the available space and power to conduct the experiments in microgravity.

Another important issue is the existence of vibration in space that can influence the results. The benefits of microgravity environment on ISS platform could be altered by gjitter. As discussed previously, the cause of these residual acceleration and vibration could be aerodynamic drag, non-ideal free falling, trembling of space vehicle, onboard machinery, and crew activities. The presence of high frequency oscillations in the spectrum of g-jitter could significantly affect diffusion experiments. Such oscillations generate timeaverage convection flows which may disturb diffusion heat and mass transfer. The effect might not be negligible for such long duration experiments that involve diffusion driven phenomena. For this matter, there are many researches done to study the effects of vibration on thermodiffusion measurements in space.

\subsubsection{Experiments to Study the Impact of Residual Acceleration}

\subsubsection{On aboard the orbital station "MIR" by the convection sensor "DACON"}

This is one of the first experiments studying the impact of residual acceleration on board a spacecraft that was performed by Russian researchers. The experiment was performed to study convection in differentially heated cylindrical cavities where 
temperature needed to be monitored and recorded at several fixed points. The results of measurements of convection and evaluation of low-frequency microaccelration components in different modules and for various regimes of crew and station activities were reported. Nevertheless, obtained results do not indicate clear evidence of time-averaged flows and related heat transfer caused by periodic high frequency g-jitter [38].

\subsubsection{MEPHISTO data from USMP-3 space flight mission}

The experiments were carried out under microgravity conditions within the framework of the MEPHISTO programme in 1996. The data obtained from this experiment represented the first quantitative data on g-jitter effects on a directional solidification experiment that were obtained under well-defined conditions. However, it was concluded that there is a lack of well-documented experimental results that can be used for verification of numerical modelling to provide reliable predictions of g-jitter effects [39].

\subsubsection{Influence of g-jitter on solute diffusion coefficient on-board the MIR station}

This experiment was dedicated to study the effect of g-jitter on the measurement of precise solute diffusion coefficients in dilute liquid metals and metalloids. The experiment was carried out on-board the MIR station using the Canadian MIM facility [40]. Their primary analysis indicated that diffusion coefficients in dilute binary metallic alloys depend upon the residual accelerations and the quality of microgravity. Further to reanalysis of this study, it was claimed that very limited number of data were considered for post-flight analysis and the results from the liquid metal diffusion experiments conducted on MIR do not support the conclusion drawn [41].

\subsubsection{IVIDIL Experiment on board ISS}

In the frame of the ESA physical Sciences project "Diffusion and Soret Coefficient Measurements for Improvement of Oil Recovery" or DSC, the experiment IVIDIL was 
planned to examine the influence of vibration stimuli on the diffusive phenomena. More specifically, it was aimed at utilizing the International Space Station for investigating the effects of vibrations on liquid diffusion and thermodiffusion. The experiment has been performed in 2009-2010 on board the ISS. The project started in October 2009 and completed in January 2010 This project of European Space Agency (ESA) gathered together European, Canadian and Russian researchers with complementary skills to prepare and carry out the experiment, to process the raw data and perform numerical modelling of the phenomena.

The first objective of this experiment was to identify the limit level of vibration below which g-jitter does not impact the experiments onboard ISS. Second objective was to perform precise measurements of diffusion and thermo-diffusion coefficients for two binary mixtures in the absence of buoyant convection. The measurements values can be used as standards for ground experiments. The third and the main objective was to study heat and mass transfer under vibration [27]. As mentioned before, many reasons, such as experiment operation, aerodynamic drag, gravity gradient, life-support systems, equipment operation crew activities, and rotational effects as well as the vehicle structural resonance frequencies provide the microgravity vibration onboard the ISS [42].

The three international teams involved in the preparation of the experiment were as follow: Microgravity Research Centre of ULB (Brussels, Belgium) team led by V. Shevtsova. This team was responsible for coordination of the entire project, all aspects related to IVIDIL experimental definition and guidance of numerical modelling. The Russian team from the Institute of Continuous Media Mechanics UB RAS (Perm, Russia) led by T. Lyubimova and team from Ryerson University led by Z. Saghir (Toronto, Canada) provided theoretical and numerical support. Each team had experience with calculation of convection flows under vibrations. Several numerical studies were carried out prior to lunching the experiment on board ISS. All the teams contributed in new findings through numerical and theoretical studies to compare with the upcoming experimental results. Shevtsova et al. studied the effect of residual acceleration on the flow 
in a side-heated rectangular cell. A new technique has been suggested based on their study to capture essential characteristics of the convective flow [43]. Chacha et al. investigated thermal diffusion in a binary mixture of methane and n-butane subject to g-jitters with moderately high frequency. They found that g-jitter causes mixing and influence the Soret effect in the cavity for various configurations. Moreover, the flow was found to response synchronously to the oscillatory accelerations. Furthermore, Chacha and Saghir studied the effect of the time-dependent vertical gravity vector on the mass diffusion in a binary fluid mixture, which is subject to a lateral temperature gradient in a rectangular cell. Their numerical study showed that g-jitter reduces the compositional variation due to the increased convection. Their results also revealed that the Soret number oscillates with time at the same frequency as the original excitation [44].

In addition to the initial numerical and ground base experimental studies, the effects of vibration which are recorded onboard ISS are studied in detail by Ahadi et al $[42,46]$ and Shevtsova et al [45]. It is found that as soon as system faces the microgravity perturbations the experimental points start to deviate from the theoretical curve and exhibit larger scattering. Shevtsova has performed several studies showing the effects of controlled vibration on the mixture by measuring different physical quantities [45]. Her investigation indicates an improvement in the understanding of the kinetic mechanisms that drive diffusion as well as effects of the g-jitters on molecular and thermal diffusion processes.

In general, the IVIDIL project has been insightful in understanding the complex process of thermal diffusion in the presence of controlled vibrations. A detailed study of the IVIDIL project is still in progress. 


\section{CHAPTER 2 SPACE EXPERIMENTAL SETUP}

The very early purpose of the IVIDIL experiment was to measure diffusion coefficients in binary mixtures under different vibrational frequencies and amplitudes. A non-uniformly concentration should be created in the liquid to fulfil the purpose of this experiment. This might diffuse during waiting time of lunch site on the Earth. Soret effect produces concentration gradient in a uniform mixture as a result of applied temperature gradient. Thus the Soret effect will be employed for creating a concentration profile across the cell [30]. This is to expand the idea of experiment to just the measurement of Soret coefficient. The reason of such idea is the driven interest of both petroleum industry and scientifically institutions.

\subsection{Pre-flight, In-flight, and Post-flight Activities}

Activities that are involved in the IVIDIL experiment need to be broken down prior to explaining the detail of each aspect of experiment. Operation of IVIDIL could be divided in to three sub-activities: Pre-flight activity, In-flight activity, and Post-flight activity.

\subsubsection{Pre-flight activities}

This stage involved extensive ground-based experiments prior to the flight to establish and improve technique for image processing. Parabolic flight tests were employed to qualify performance of suggested approaches for IVIDIL microgravity conditions. Also, numerical simulations of all experimental runs were prepared to compare with the experimental results.

\subsubsection{In-flight activities}

This stage involved keeping track of all the generated data and control of housekeeping data. Also, the crew were required to process the raw images as fast as possible and send 
them to laboratories for analysis. They needed to take necessary action and fix any problems that appeared. Here is an example of teleconference from ISS: "we have found that side and direct view are symmetric in the cases when it has to be. Urgent negotiation with Verharert (Qinetiq) and Lambda_x identify the problem: different optical path. The decision was taken- to preheat system during about 6 hours after the switching off" [43]. Moreover, continuous teleconferences were setup to discuss the on-going progress.

\subsubsection{Post-flight activity}

This step involves saving and processing numerous raw data. A suitable model needs to be elaborated and results need to be analysed. This study is an example of what has been done in terms of post-processing of IVIDIL experiment.

The aim of this chapter is to provide all aspects of IVIDIL experiment that is setup on board ISS. A detailed review of experimental apparatus along with a step by step procedure of this unique experiment is illustrated. The experimental substances and their physical properties are introduced. Additionally, the strategy of experiment and the method of data extraction are described in details.

\subsection{Experimental Apparatus (IVIDIL Instruments)}

The IVIDIL experimental apparatus was placed inside $\mathrm{ESA}^{3} \mathrm{SODI}^{4}$ multi-user facility which, in turn, was installed in a special facility known as MGS ${ }^{5}$. This facility offers an enclosed 255-litter (9 cubic foot) work area accessible to the crew through glove ports and to ground-based scientists through real-time data links. Because the work area is sealed and held at a negative pressure, the crew can manipulate experimental hardware and samples without the danger of small parts, particulates, fluids, or gasses escaping into the open laboratory module. The MGS is located in the European Space Agency's Columbus Orbital

\footnotetext{
${ }^{3}$ European Space Agency

${ }^{4}$ Selectable Optical Diagnostics Instrument

${ }^{5}$ Microgravity Science Glovebox
} 
Facility (COF) laboratory module. Figure 2-1 is a picture of IVIDIL experimental apparatus mounted in the MGS.

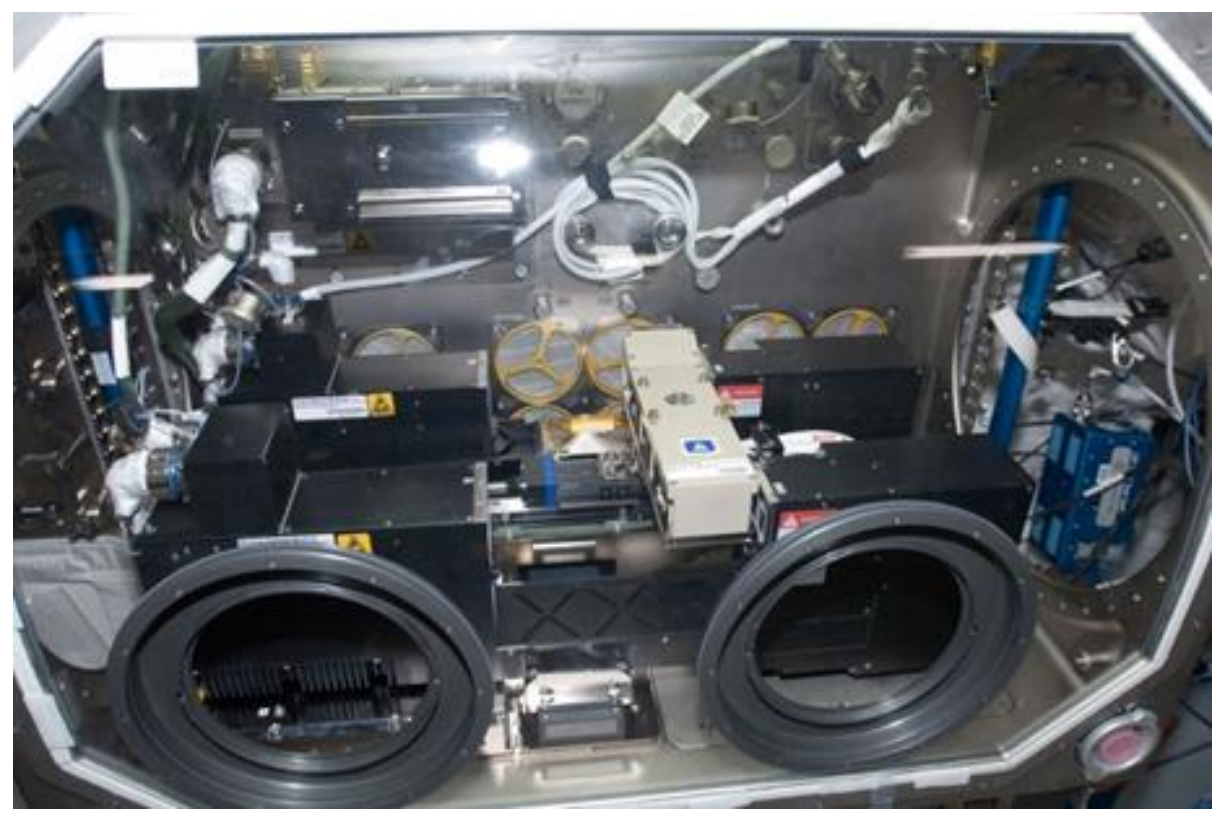

Figure 2-1: IVIDIL inside Glovebox at the ISS [45]

Initially, SODI was developed as a multi-purpose facility for conducting three experiments: IVIDIL, COLLOID, and DSC. The hardware development was provided by QinetiQ Space NV (Kruibeke, Belgium) and optical development was provided by Lambda-X (Nivelles, Belgium) [45].

The IVIDIL apparatus consists of three main parts: An interferometric system in combination with equipment for digital recording the phase information; a diffusion cell and a vibrational stimulator.

\subsubsection{Interferometry}

The setup for the measurement of the Soret effect by ODI ${ }^{6}$ has been developed by ULB and undergone several tests against benchmark mixtures. The optical part is designed based

${ }^{6}$ Optical Digital Interferometry 
on Mach-Zehnder interferometer for observing the spatial and temporal variations of composition in the mixture. Figure 2-2 shows implemented version of the interferometer in combination with equipment for digital recording the phase information. The laser beam of constant frequency He-Ne with wavelength $\lambda=670 \mathrm{~nm}$ is expanded by a spatial filter and then passes through the beam splitter $\left(\mathrm{BS}_{1}\right)$ where it is divided into two beams of equal intensity. One of them goes through the cell holder and the experimental cell; the other one bypasses the cell. The external walls of the cell are shaped in the form of two prisms to allow optical observation in two perpendicular directions. Figure 2-3 shows two different paths inside the cell holder. One of them leads the beam through the front of the cell

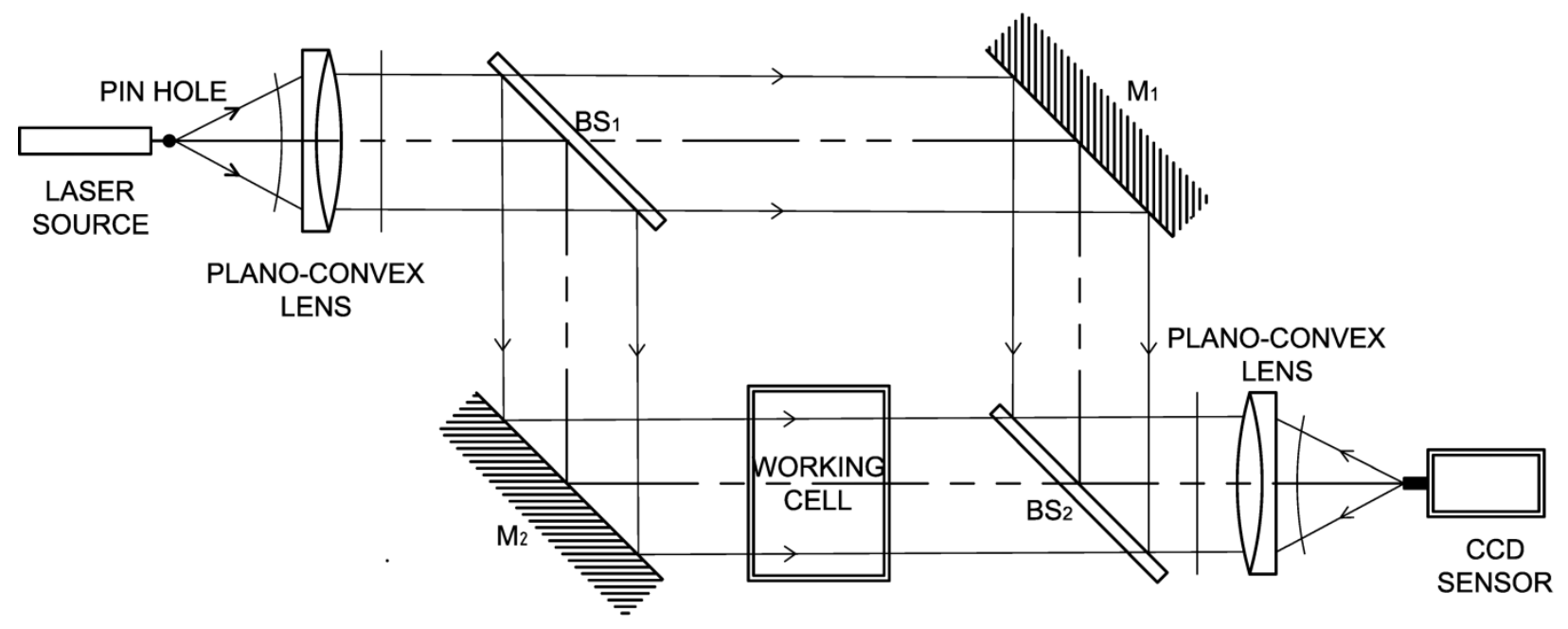

Figure 2-2: Scheme of the Interferometer Setup

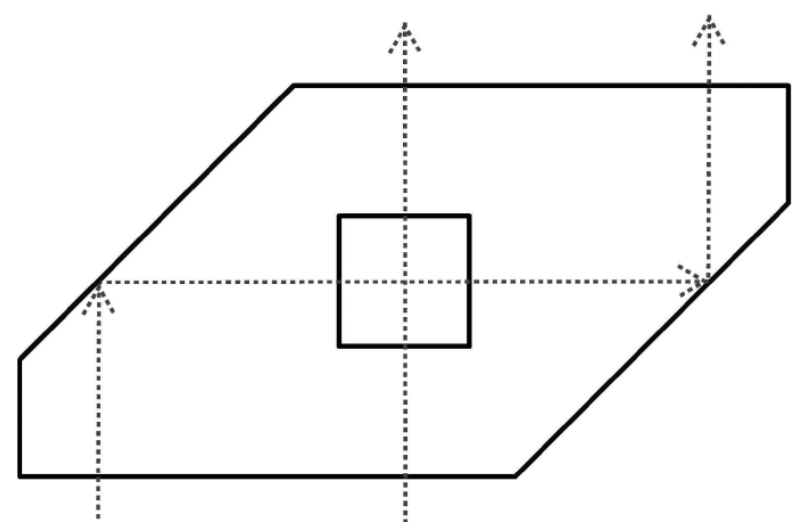

Figure 2-3: Top view of the experimental setup [45] 
(direction perpendicular to the thermal gradient and force vibration) and the other one through its side (along vibration direction). After passing mirrors the object and reference beams interfere with each other at the second beam splitter $\left(\mathrm{BS}_{2}\right)$. Finally interference fringes are captured by a Charge Coupled Device, CCD, camera 1280 x 1024 pixels sensors [30]. An example of interference pattern in the cell is shown in Figure 2-4. The image on the left refers to the interference pattern in front view of the cell. This view is used for the purpose of this study to obtain the concentration difference in the cell. Whereas, the image on the right side of Figure 2-4 shows the side view of the cell. The main reason to provide the particular view is to further extend studies in $3 \mathrm{D}$ and to study velocity of particles in the mixture. In other words, the availability of two perpendicular views of the cell allows determining three-dimensional coordinates of the tracer particles. These tracers are hollow ceramic spheres with radius $75 \pm 20 \mu m$ [45].

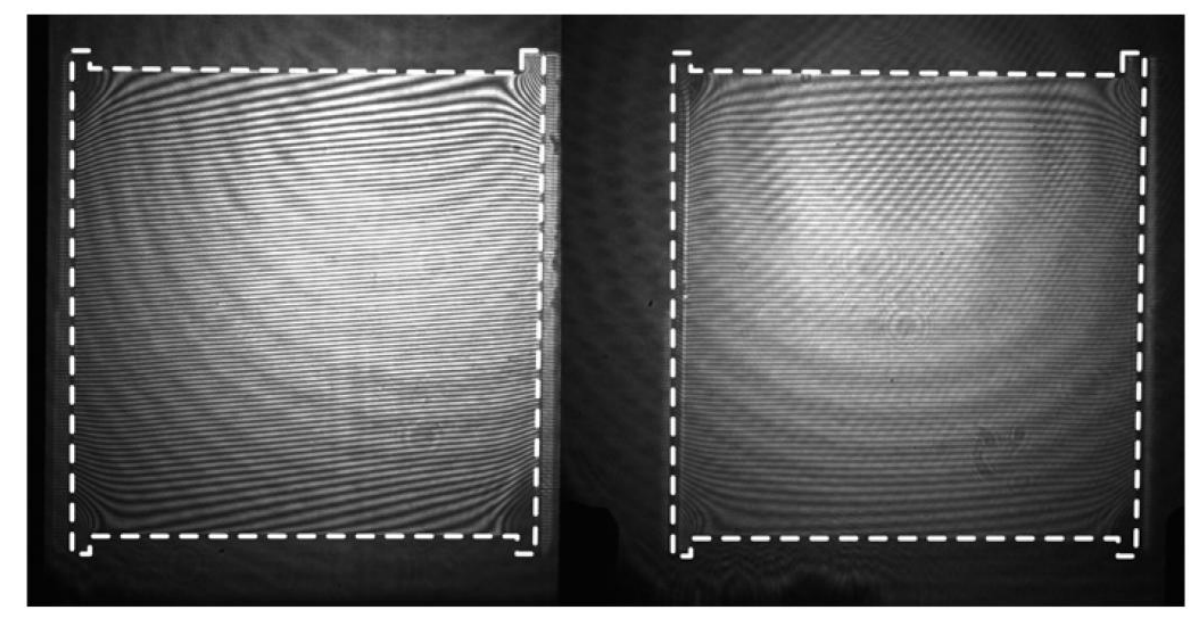

Figure 2-4: Typical Interferogram from the IVIDIL experiment [27]

\subsubsection{Diffusion cell}

The initial idea of the experimental cell design is to have a linear temperature distribution inside liquid when heating is applied to the cell. All the technical points need to be inspected before proceeding to the temperature analysis inside experimental cell. Figure 2-5 illustrates the cell geometry and its structure used in this study. 


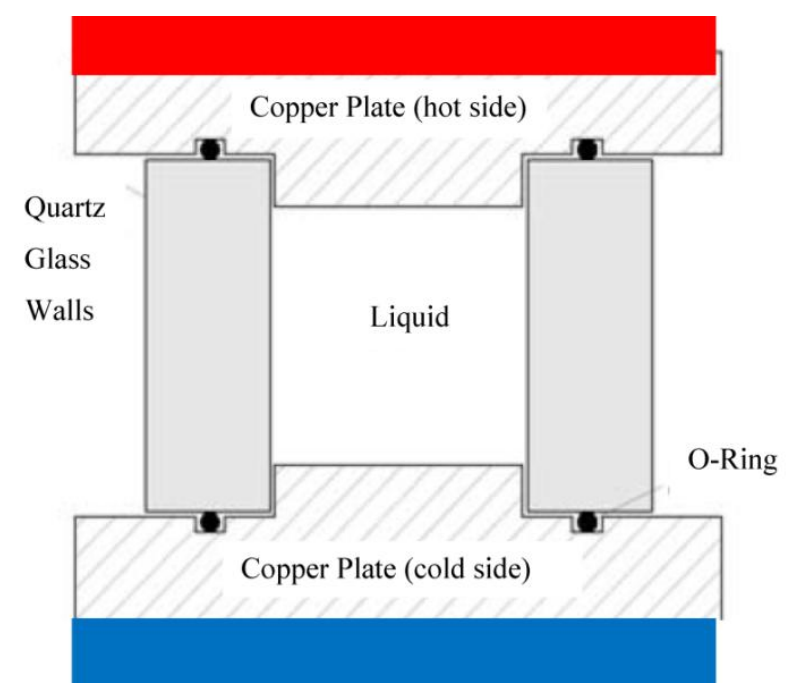

Figure 2-5: Experimental Cell [13]

\subsubsection{Cell design}

The diffusion cell is made of quartz Suprasil by the Hellma Company with an internal size of $\mathrm{L}=10 \mathrm{~mm}[30]$. The cell configuration is selected based on an optimization analysis on different aspect ratio of Soret cell [47]. Mainly, the reason for this particular cell size is to avoid convection inside the cell and to have linear temperature distribution inside the test liquid [47]. The temperatures inside pure water and glass wall are measured and results are shown in Figure 2-6.

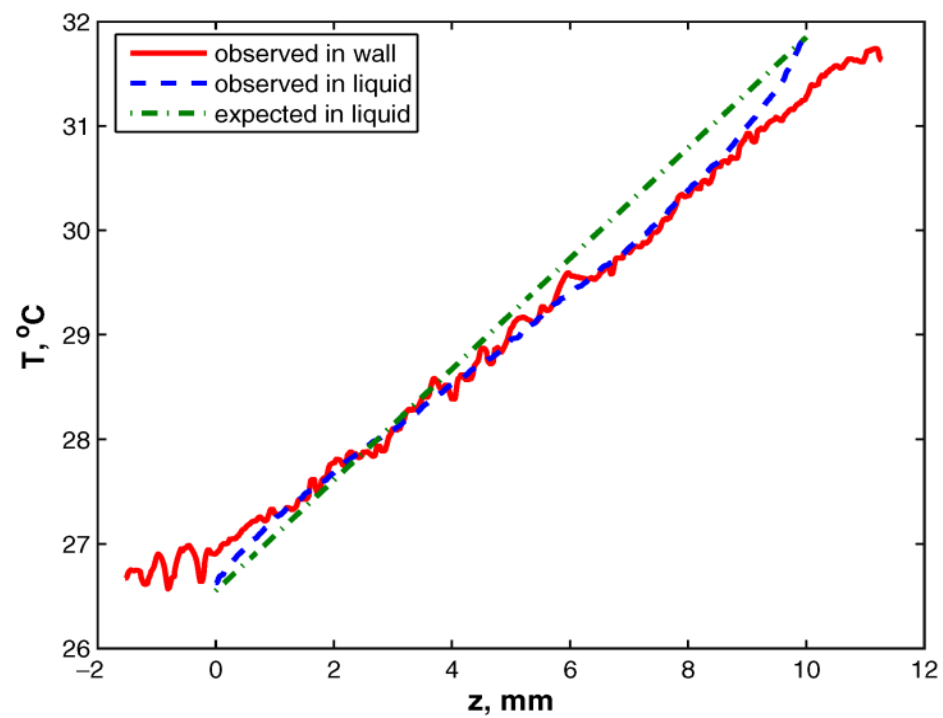

Figure 2-6: The vertical temperature profiles: inside glass wall, inside liquid (water) and expected linear behaviour [47] 
The dashed and solid lines represent vertical temperature profiles inside the liquid and inside the quartz glass which were measured $0.5 \mathrm{~mm}$ away from the edge of the glass itself. It can be seen that the measured temperature do not coincide with the expected linear profile that is given by dashed-dotted line. It can be shown that both experimental profiles behave in similar fashion along the major part of the cell except the bottom and top corners. At the top of the cell, the liquid is hotter than the glass, while at the bottom the situation is opposite. The disagreement near the top and bottom of the cell illustrates the presence of lateral heat flux [47]. The reasons could be different heat conductivities of contact materials, thermal distribution by entire geometry of set-up, and heat transfer through external side of lateral walls.

\subsubsection{Thermal design}

Moreover, the thermal design of the cell is an important factor beside its geometry. Special care needs to be taken while designing a cell because inaccurate thermal design can completely discard the advantages of Interferometry technique. The reason is that thermal design of the cell determines the presence and intensity of the residual convection inside the cell [47]. Besides, inaccurate design of the cell would cause appearance of bubbles and undesirable thermal gradient. In a long duration experiments such as IVIDIL, appearance of bubbles is a dangerous phenomenon because it may cause convection as bubbles move to the hot side initiating near-by flow in the cell. As a result convection can appear at the lateral walls of the cell and in the corners [47].

Shevtsova et al. discuss about the cell size and shape optimization as well as importance of its thermal design in their recent work [45]. Firstly, they emphasize on the presence of convection flows in diffusion cell that significantly alter the concentration field. Secondly, they highlight the accuracy of thermal design of the cell that could greatly influence the advantages of interferometry. They overcome the issue of convection inside the liquid near the corners of the cell by using cell with larger aspect ratio and preventing leakage of the liquid. Nevertheless, the cell is changed for the new series of thermodiffusion experiment 
onboard ISS due to some technical problems of the cell. The new configuration induces non-linear variation of temperature and provides a suitable condition to have convection cell by external disturbances such as g-jitter vibration. Flat copper plates cover not only the liquid but also the glass walls. Sealing between glass and copper is made of material with thermal conductivity close to that of glass. This design would greatly reduce the horizontal temperature gradient near the walls.

\subsubsection{Vibration stimuli}

The cavity and its holder are fixed to a linear motor that performs translational harmonic oscillation vibration perpendicular to the temperature gradient. As a result of vibrations and a non-uniform temperature field, specific flows appear. These flows are called thermo-vibrational convection, which are caused by different inertia of cold and hot parts of the fluid with respect to the vibration acceleration [43]. In other words, high frequency vibration leads to appearance of time-average flows (or streaming flows), which can essentially affect heat and mass transfer processes. The thermo-vibrational convection is most pronounced in the absence of other external forces in particular, static gravity [27]. These vibrational flows are characterized by the vibrational analogue of Rayleigh number (Rayleigh vibration or Gershuni number) [48].

$$
R a_{v i b}=G s=\frac{\left(A \omega \beta_{T} \Delta T L\right)^{2}}{2 v \chi}
$$

Where $A$ is the amplitude of vibrations, $\omega=2 \pi f$ is the angular frequency, $L$ is the characteristic size (i.e. optical length), $\Delta T$ is the applied temperature gradient, is $\beta_{T}$ the thermal expansion coefficient, $v$ is the kinematic viscosity and $\chi$ is the thermal diffusivity.

In the experiments, the frequency and amplitude are varied in the ranges $0.5-2.8 \mathrm{~Hz}$ and $10-61 \mathrm{~mm}$, respectively. The maximum vibrational acceleration $A \omega^{2}=0.998 g_{o}$ is achieved at $A=61 \mathrm{~mm}$ and $f=2 \mathrm{~Hz}$ [45]. The upper limit of frequency increases as the amplitude decreases. 
The experimental cell is placed in a cell array containing two identical optical cells. The dimensions of the cell array are $8.5 \mathrm{~cm}(h) \times 8 \mathrm{~cm}(W) \times 30.5 \mathrm{~cm}(L)$. Figure 2-7 shows directions of temperature difference and control vibration which is placed inside the cell array. The two identical cells are filled with the same liquid and are used for two different purposes. The one on the left is called primary cell that contains only liquid. The images taken from this cell are used for the purpose of studying thermodiffusion. The cell on the right, refers to Figure 2-7, is called companion cell and contains tracer particles in addition to the liquid mixture. The main purpose of filling the companion cell with tracer particles is to analyse the convective flow and velocity of flow in the cell.
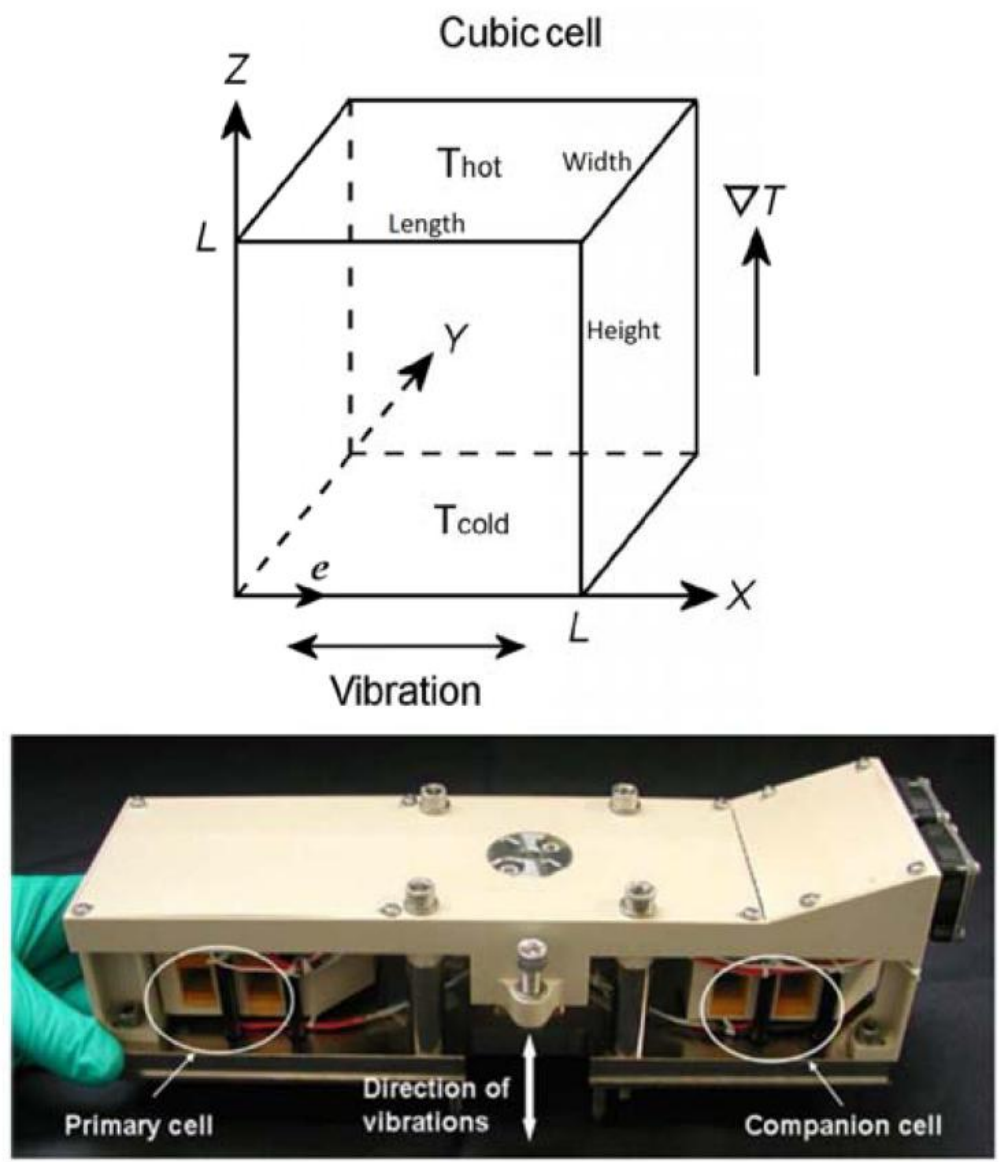

Figure 2-7: Cubical cell and core part of IVIDIL instrument; by courtesy of Verhaert Space [30] 


\subsection{Experimental Procedure}

Two different concentrations of Water-IPA $^{7}$ respectively with positive and negative Soret effect are chosen as test fluids. The IVIDIL experiment included two cell arrays. One is dedicated to mixture with negative Soret coefficient that is water (90\%)-Isopropanol (10\%). The other one contains the mixture of water (50\%)-Isopropanol (50\%) with positive Soret coefficient. As previously mentioned, each cell array included two cells: a primary and a companion for different purposes. The cell arrays were exchanged on December, 2009 [45]. The physical properties of liquids are given in Table 2-1.

\begin{tabular}{|c|c|c|c|c|c|c|c|}
\hline & $\begin{array}{c}\rho \\
\mathrm{kg} / \mathrm{m}^{3}\end{array}$ & $\begin{array}{c}\beta_{T} \\
10^{-4} \mathrm{~K}^{-1}\end{array}$ & $\beta_{C}$ & $\begin{array}{c}v \\
10^{-6} \mathrm{~m}^{2} / \mathrm{s}\end{array}$ & $\begin{array}{c}\chi \\
10^{-7} \mathrm{~m}^{2} / \mathrm{s}\end{array}$ & $\begin{array}{c}D \\
10^{-10} \mathrm{~m}^{2} / \mathrm{s}\end{array}$ & $\begin{array}{c}S_{T} \\
10^{-3} 1 / K\end{array}$ \\
\hline $\mathrm{H}_{2} \mathrm{O}(90 \%)-I P A(10 \%)$ & 984 & 3.1 & -0.14 & 1.41 & 1.3 & 7.4 & -7.08 \\
\hline $\mathrm{H}_{2} \mathrm{O}(50 \%)-I P A(50 \%)$ & 905 & 7.7 & -0.25 & 3.10 & 0.85 & 1.62 & 5.45 \\
\hline
\end{tabular}

Table 2-1: Physical properties of the mixtures at $25{ }^{\circ} \mathrm{C}$

In total, 55 experimental runs (41 original and 14 re-runs) were successfully conducted by January 2010. Each original run lasted 18 hours and all of them were controlled via telescience provided by the Spanish User Support Centre (E-USOC, Madrid).

First step is to initialize mixture characteristics by reaching uniform concentration and temperature inside the cavity. Thus the cell array is shaken for a period of time for the first installation; then to stabilize the temperature of the cell and interferometer at a certain temperature, which the process lasts approximately 4 to 6 hours [45]. Figure 2-8 shows the initial preparation step by the astronaut. The mean temperature that is established at the top

\footnotetext{
${ }^{7}$ The abbreviation form of Isopropanol
} 


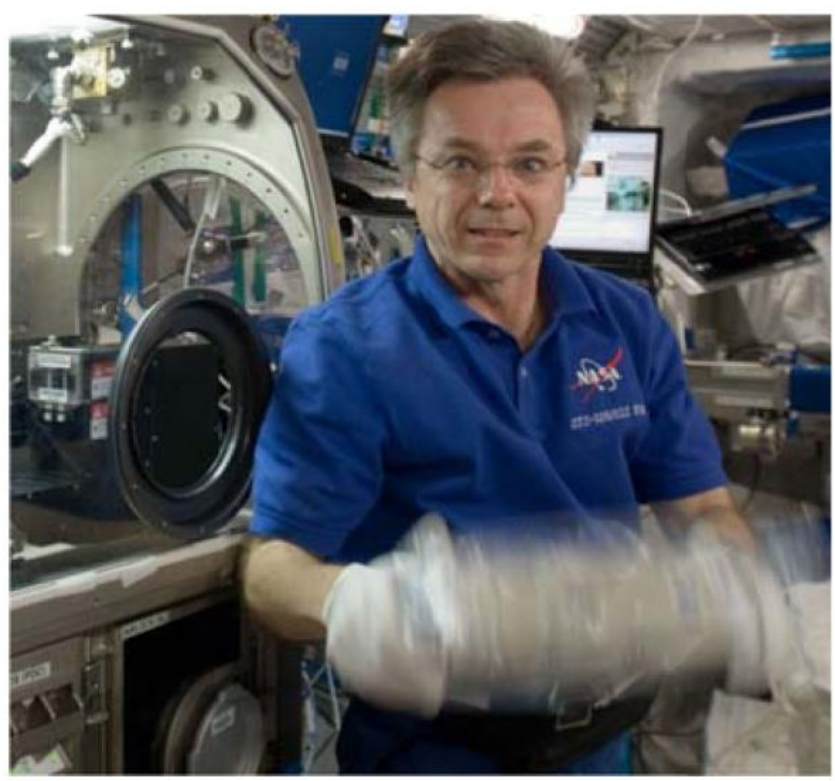

Figure 2-8: Canadian Space Agency Astronaut Bob Thirsk prepares the cell array [45]

and bottom plates of the cell is $25^{\circ} \mathrm{C}$. Second step is to apply thermal gradient over the cell. This temperature difference in the liquid bulk causes phase variation. The thermal equilibrium is established on a characteristic time which is a function of length of the experimental cell and thermal diffusivity $\tau_{t h} \propto\left(L^{2} / \chi\right)$; during this period the influence of diffusion due to thermal difference on refractive index is so small because the effect of thermal contrast factor is two orders of magnitude larger than concentration contract factor [45]. Thus, any change in refractive index is assumed to be due to temperature in this period. Eventually, a concentration gradient is established by continuing to impose temperature gradient across the experimental cell to a uniform mixture. As result of Soret effect, concentration profile is slowly generated in initially homogeneous binary mixture. Also, there is a relaxation factor for the diffusion process like the thermal time at the beginning. Change in refractive index in this period is effected by both temperature and concentration. This factor is function of length of the cell and diffusion coefficient. Diffusion time or relaxation factor is about 12 hours for our specific mixture and special geometry of our cell and is calculated by the following relation: $\tau_{D}=\frac{L^{2}}{D} \approx 12 h$ [45]. This is 
the time that separation of species is expected to be stabilized in the mixture. Conversely, some might argue that 12 hours is not enough to reach steady state condition for separation of this mixture considering noticeable amount of separation during the last hours of experiment onboard ISS. Figure 2-9 is a conflicting example showing the maximum separation for Run Ext1 which is continued for 22 hours. However, it should be noted that approximating the duration of experiment is a compromise between relaxation time and available microgravity time [30]. This means the 12 hours duration of non-isothermal step is sufficient to approach steady state.

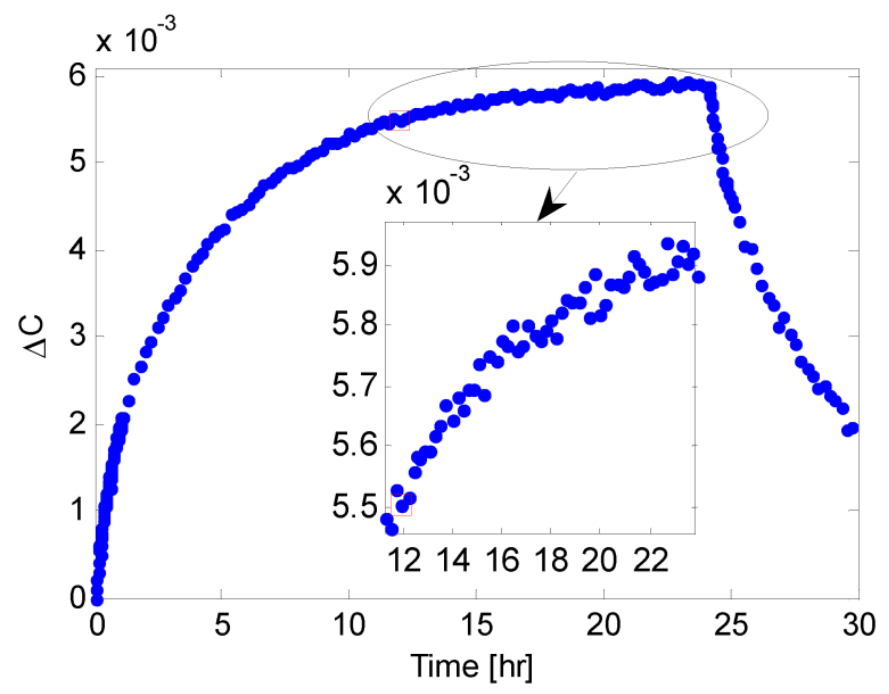

Figure 2-9: Concentration profile for Run 2 IVIDIL [46]

After $12 \mathrm{~h}$ the temperature gradient is removed and pure diffusion occurs during 6 hours. After similar thermal time for the second phase, any changes in refractive index is due to concentration during this period. In the 6 hours period the system is reverted to an isothermal case where again the temperature is kept constant at $T_{\text {mean }}=25^{\circ} \mathrm{C}$ [45]. Vibration of different amplitude and frequency are applied during these 18 hours.

In this study, several parameters such as temperature and concentration fields in the cavity are studied. Digital optical interferometry provides large view over the entire cell and therefore allows identifying the convection free zones as well as temperature and concentration distribution in the cell at any location. 
In total eight experimental runs are selected and can be seen in Table 2-2. The first four runs ( 1 to 4 ) according to Table $2-2$ are selected from the mixture of (10\% IPA-90\% Water) with negative Soret coefficient. $\Delta \mathrm{T}$ is kept at constant value of $5 \mathrm{~K}$ for all the runs in this set. Conversely, frequency and amplitude of applied vibration vary in each run. The main objective is to not only investigate any possible effect of vibration on measurement of Soret coefficient but to evaluate the quality of those effects under different Rayleigh vibration levels.

\begin{tabular}{|c|c|c|c|c|c|}
\hline RUN\# & $\mathbf{c}_{\mathbf{0}, \text { IPA }}$ & $\Delta \boldsymbol{T}\left(25 \pm \frac{\Delta \boldsymbol{T}}{2}\right)$ & $\boldsymbol{f}[\boldsymbol{H z}]$ & $\boldsymbol{A}[\boldsymbol{m}]$ & $\boldsymbol{R} \boldsymbol{a}_{\text {vibration }}$ \\
\hline Run 1 & $10 \%$ & 5 & 0.5 & 0.070 & 31.69 \\
\hline Run 2 & $10 \%$ & 5 & 0 & 0 & 0 \\
\hline Run 3 & $10 \%$ & 5 & 2.0 & 0.025 & 64.68 \\
\hline Run 4 & $10 \%$ & 5 & 1.0 & 0.050 & 64.68 \\
\hline Run 32 & $50 \%$ & 15 & 0.5 & 0.070 & 908.11 \\
\hline Run 34 & $50 \%$ & 5 & 0.5 & 0.070 & 100.90 \\
\hline Run 36 & $50 \%$ & 10 & 0.5 & 0.070 & 403.60 \\
\hline Run 38 & $50 \%$ & 10 & 0.1 & 0.070 & 16.14 \\
\hline
\end{tabular}

Table 2-2: Selected IVIDIL runs and properties

The second group of runs $(32,34,36$, and 38) according to Table 2-2 are selected from the mixture of (50\% IPA-50\% Water) with positive Soret coefficient. These runs have same amplitude and frequency of vibration except run 38. Conversely, temperature difference varies in each run. It should be noted that the amount of experimental information and benchmark values for mixtures with positive Soret separation ratio are noticeably less than the one with negative Soret separation ratio. In other words, the behaviour of mixture with positive Soret separation ratio is further investigated. 


\subsection{Raw Image Data}

Data are received from ISS microgravity laboratory in format of raw images. The format and the naming convention need to be discussed before getting into analysis stage.

Data that are directly from the camera or after processing by the SODI ASW ${ }^{8}$ is stored into the SODI removable hard drive as individual files. The naming format of these files is EE_yy_XXXX@1234_LS_typ_YYMMDD_HHMMSS_\#.ext. Table 2-3 shows the detail description of each section of the files name have specific meaning. For example: 110 0000@0000 FR DSC 090117102518 4.raw is an image that is captured while cell array with ID 11 is installed from the fixed camera so cell 0 . Then, there is no active timeline (0000@0000). The Red Laser source is used in the Fixed Optics and the raw image data is processed into a $\mathrm{DSC}^{9}$ image, which on inspecting give the following image:

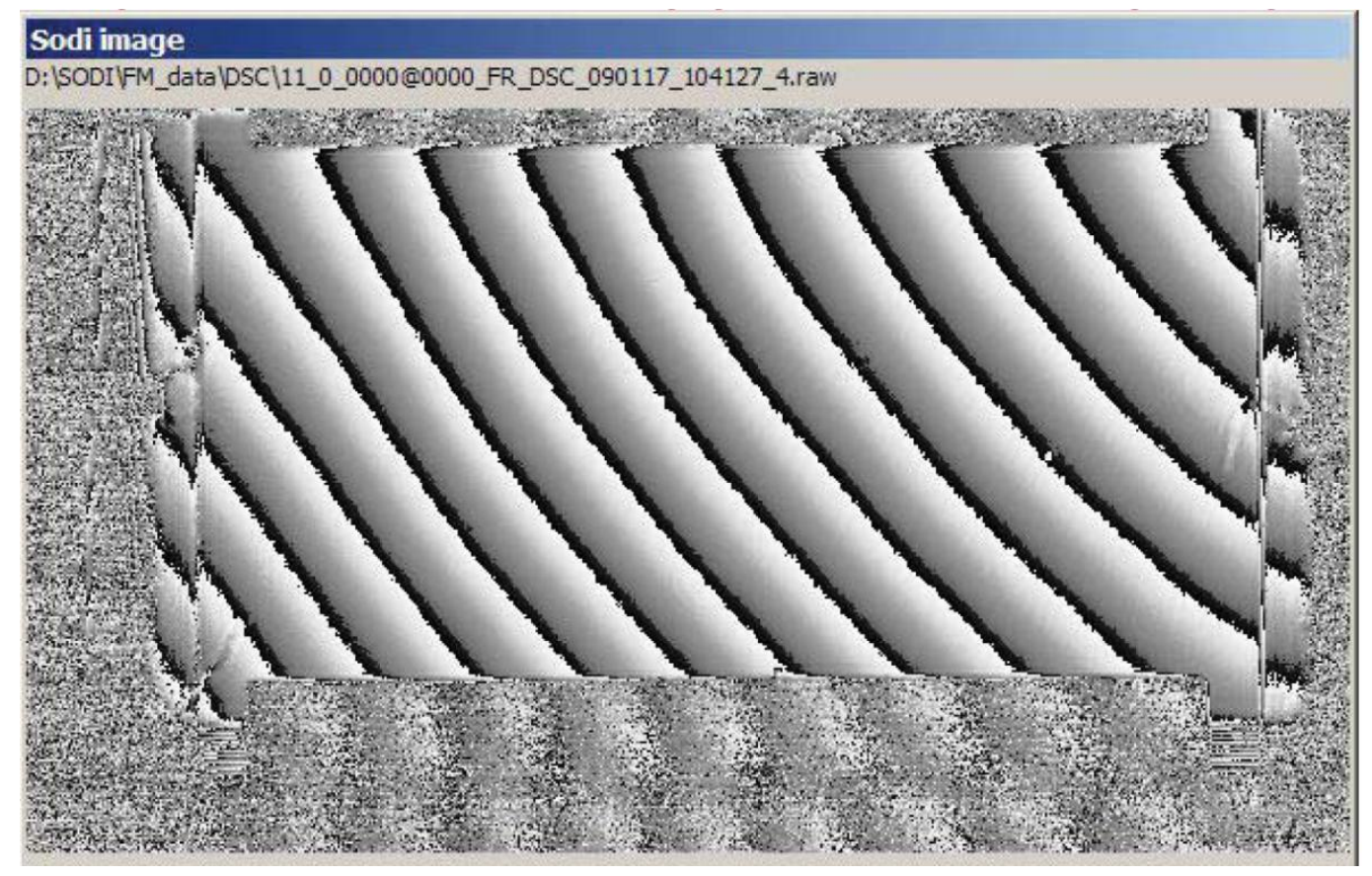

Figure 2-10: Image data after converting from RAW to BMP format [49]

\footnotetext{
${ }^{8}$ Abbreviation for selectable optical diagnostics Instrument Application Software

${ }^{9}$ Abbreviation for Diffusion and Soret Coefficients (experimental cell)
} 


\begin{tabular}{|c|c|c|}
\hline Field & Field Name & Range \\
\hline$E E$ & Experiment ID & $0-15$, with $15=$ No cell array present \\
\hline yy & Cell ID & $\begin{array}{l}\text { Theoretically a number in the range of 0-99 } \\
0=\text { unknown/no cell array }\end{array}$ \\
\hline$X X X X$ & $\begin{array}{l}\text { Timeline Script } \\
\text { checksum, in } \\
\text { hexadecimal } \\
\text { notation }\end{array}$ & $\begin{array}{l}\text { The Timeline Script Checksum is calculated at the time of } \\
\text { script loading }\end{array}$ \\
\hline 1234 & $\begin{array}{l}\text { Timeline step } \\
\text { counter }\end{array}$ & $\begin{array}{l}\text { Identifies the current Timeline Script, tracing the image } \\
\text { back to the script }\end{array}$ \\
\hline$L S$ & Light Source & $\begin{array}{l}\text { FR= Red laser in Fixed optical Bridge } \\
\text { MR=Red laser in Movable optical Bridge }\end{array}$ \\
\hline Typ & Type of Image & $\begin{array}{l}\text { RAW= raw image from camera } \\
\text { DSC=DSC scientific image } \\
\text { SUB=result of two images being subtracted } \\
\text { MULT=individual pixels in two images multiplied by } \\
\text { each other }\end{array}$ \\
\hline$Y Y M M D D$ & Date Stamp & \\
\hline HHMMSS & Time Stamp & \\
\hline \# & Sequence Number & $\begin{array}{l}\text { When multiple time stamps are generated within one } \\
\text { second, a sequence number is added, incremented by } 1 \\
\text { with each image stamp generated. } \\
\text { Note that not all image data is recorded to disc, so these } \\
\text { sequence numbers may or may not be continuous. }\end{array}$ \\
\hline Ext & Extension & $\begin{array}{l}\text { Default is 'raw': } \\
\text { image_heigt*image_width*pixe_size( } 8 / 16 \text { bits) } \\
\text { consecutive bytes }\end{array}$ \\
\hline
\end{tabular}

Table 2-3: Description of raw image naming convention [49] 
Table 2-3 is a modified version of a detailed data table taken from SODI-IVIDIL Software manual [49]. However, only some part of this information is needed for data analysis of this study. The most important parts are the date, time, cell number, image sequence, and type of the laser used in the experiment. The date and time clearly indicate which image should be taken for start of experiment, or when temperature is applied and so on so forth. The available images for this study illustrate two types of laser which are MR and FR. For IVIDIL the fixed red laser (FR) is used for the companion cell (refer to Figure 2-7). On the other hand the moving red laser (MR) is used for the cell with Water-IPA mixture. Thus, only the images of the moving red laser (MR) are separated and used for this study. Moreover, this laser takes a set five consecutive images every 20 second. So, the sequence number at the end of the image file name indicates whether it is image $1,2,3,4$, or 5. As for data provided for this study, the sequence numbers are mostly 1 . Furthermore, the first two letters (i.e. EE) that represent experimental ID and consist of two digits ranging from 0 to 15 are linked to cell array. In this study only one cell array is used; therefore, the experimental ID does not change. It should be noted based on Table 2-3 the numbers of images are different for each runs because not all the images are recorded to the disc and sent for processing. This would impact the accuracy of image processing and getting exact result for each instances of the experiment. 


\section{CHAPTER 3 IMAGE PROCESSING TECHNIQUE}

The procedure established for image processing is as follow: Each interferogram is first subjected to resizing or cropping the image to remove crashed pixels around the walls. Then it is reconstructed by performing a two-dimensional Fast Fourier Transform of the fringe image, filtering a selected band of the spectrum that includes one of non-zero peaks of the centre of the band, replace this band at the middle of the spectrum, and performing an inverse 2D Fast Fourier Transform of the filtered result, and phase unwrapping. Then the gradients of composition inside of the fluid are calculated from refractive index gradient and local temperature for steady regimes. The mathematical description of above steps is shown in this section.

\subsection{Phase Extraction from Fringe Image}

The measured intensity distribution $i(x, y)$ can be written in the form

$$
i(x, y)=a(x, y)+b(x, y) \cos [\phi(x, y)]
$$

Where $a(x, y)$ represents the background variation and $b(x, y)$ is related to the local contrast of the pattern. $\phi(x, y)$ is the phase of the intensity $i(x, y)$. Since the cosine is a periodic and even function, every phase calculated from one intensity distribution is indefinite to an additive integer multiple of $2 \pi$.

$$
\cos \phi=\cos (2 \pi n+s \phi), \quad n \epsilon \mathbb{Z}, \quad s \epsilon\{-1,+1\}
$$

Now, the fringe-pattern formula, equation 3-1, can be rewritten as

$$
i(x, y)=a(x, y)+c(x, y)+c^{*}(x, y)
$$

Where 
$c(x, y)=\frac{1}{2} b(x, y) \exp [j \phi(x, y)]$

With $j=\sqrt{-1}$ and $*$ denoting the complex conjugate. Now equation 3-4 needs to be Fourier transformed. This is done one-dimensionally with respect to $x$, which gives

$$
I(u, y)=A(u, y)+C(u, y)+C^{*}(u, y)
$$

or one-dimensionally with respect to $y$, which gives

$$
I(x, v)=A(x, v)+C(x, v)+C^{*}(x, v)
$$

or two-dimensionally which results to

$$
I(u, v)=A(u, v)+C(u, v)+C^{*}(u, v)
$$

since the variation of background intensity is slow compared with the fringe spacing, the amplitude spectrum will be a trimodal function with $A$ broadening the zero peak and $C$ and $C^{*}$ placed symmetrically to the origin. Next step is to filter out either of the two spectra on the carrier, for instance $C$, and translate it on the frequency axis toward the origin. Note that the unwanted background variation has been filtered out at this stage [50]. Again using Fast Fourier Transform (FFT) algorithm, the inverse Fourier transform of $C(u, v)$ with respect to $u$ and $v$ would give $c(x, y)$ defined by equation $3-4$. Then calculating a complex logarithm of equation 3-4 gives:

$$
\log [c(x, y)]=\log \left[\left(\frac{1}{2}\right) b(x, y)\right]+i \phi(x, y)
$$

From $c(x, y)$ the interference phase is calculated point wise by

$$
\phi(x, y)=\tan ^{-1} \frac{\operatorname{Im}[c(x, y)]}{\operatorname{Re}[c(x, y)]}
$$


Where $R e$ denotes the real part and Im the imaginary part [50]. The signs of the numerator and the denominator in equation 3-9 must be taken into account separately, which lead to values ranging from $-\pi$ to $+\pi$.

A similar procedure is applied to the reference image to evaluate $\phi_{r e f}(x, y)$. The required phase difference, $\Delta \phi$, or phase distribution is given by the difference between the phase of ith image and the very first image as a reference [51].

$$
\Delta \phi\left(x, y, t_{i}\right)=\phi\left(x, y, t_{i}\right)-\phi_{r e f}\left(x, y, t_{r e f}\right)
$$

At the next step the mean value of phase difference for all the pixels is calculated. Then, the deviation of each phase difference with respect to the mean value is calculated. This is used to determine the change of refractive index [52].

$$
\begin{aligned}
& \Delta \phi_{\text {mean }}\left(t_{1}\right)=\frac{1}{N} \sum_{i=1, N} \Delta \phi\left(x_{i}, y_{i}, t_{1}\right) \\
& \delta \phi\left(x, y, t_{1}\right)=\Delta \phi\left(x, y, t_{1}\right)-\Delta \phi_{\text {mean }}\left(t_{1}\right)
\end{aligned}
$$

\subsection{Phase Unwrapping}

The above phase difference calculated by arctangent function is wrapped and it belongs to the range $(-\pi, \pi)$. It should be unwrapped to construct the continuous natural phase. Phase unwrapping is a simple task for a one-dimensional case, while for a two-dimensional case and noisy fringes, sophisticated unwrapping techniques are required [51]. In other words, one should perform a two-dimensional evaluation to make full use of all the information contained in the whole interferogram. So, a two-dimensional unwrapping technique is used in this study. In the two-dimensional case there are several possibilities for choosing the band filter in the Fourier plane.

Nonetheless, two-dimensional phase unwrapping proceeds iteratively in the $x$ and $y$ direction to obtain full information contained in the whole interferogram. Equation 3-13 illustrates the two dimensional method that is used to unwrap the subtracted phases. 


$$
\left.\begin{array}{l}
n\left(x_{m / 2}, y_{n / 2}\right)=0, \\
n\left(x_{m / 2}, y_{i}\right)=\left\{\begin{array}{lr}
n\left(x_{m / 2}, y_{i-1}\right) & \text { if }\left|\phi\left(x_{m / 2}, y_{i}\right)-\phi\left(x_{m / 2}, y_{i-1}\right)\right|<\pi \\
n\left(x_{m / 2}, y_{i-1}\right)+1 & \text { if } \phi\left(x_{m / 2}, y_{i}\right)-\phi\left(x_{m / 2}, y_{i-1}\right) \leq-\pi \\
n\left(x_{m / 2}, y_{i-1}\right)-1 & \text { if } \phi\left(x_{m / 2}, y_{i}\right)-\phi\left(x_{m / 2}, y_{i-1}\right) \geq \pi
\end{array}\right. \\
i=\frac{m}{2}, \frac{m}{2}+1, \ldots m \text { and } \frac{m}{2}, \frac{m}{2}-1, \ldots 1 \\
n\left(x_{j}, y_{i}\right)=\left\{\begin{array}{l}
n\left(x_{j-1}, y_{i}\right) \\
n\left(x_{j-1}, y_{i}\right)+1 \quad \text { if } \phi\left(x_{j}, y_{i}\right)-\phi\left(x_{j-1}, y_{i}\right) \leq-\pi \\
n\left(x_{j-1}, y_{i}\right)-1
\end{array} \quad \text { if } \phi\left(x_{j}, y_{i}\right)-\phi\left(x_{j-1}, y_{i}\right) \geq \pi\right.
\end{array}\right\}
$$

Where $i$ and $j$ are coordinate index of pixels in $y$ and $x$ direction, respectively. $m$ and $n$ are number of pixels in $x$ and $y$ direction, respectively.

In other words, the unwrapping procedure starts from a pixel at the centre of the image. The wrapped phase of this pixel is compared with a neighbour pixel that is previously validated. If the difference between two neighbour pixel is less than $\pi$, the phase remains unchanged. If the difference is less than $-\pi$, the phase is equal to its wrapped phase plus $2 \pi$. Finally, if the difference between two pixel is more than $\pi$, the phase is equal to its wrapped phase minus $2 \pi$. At the end, a smooth $2 \mathrm{D}$ plot of phase change is obtained in the range $-\pi$ and $\pi$.

Figure 3-1 is a graphical illustration of all the above steps in image processing. 


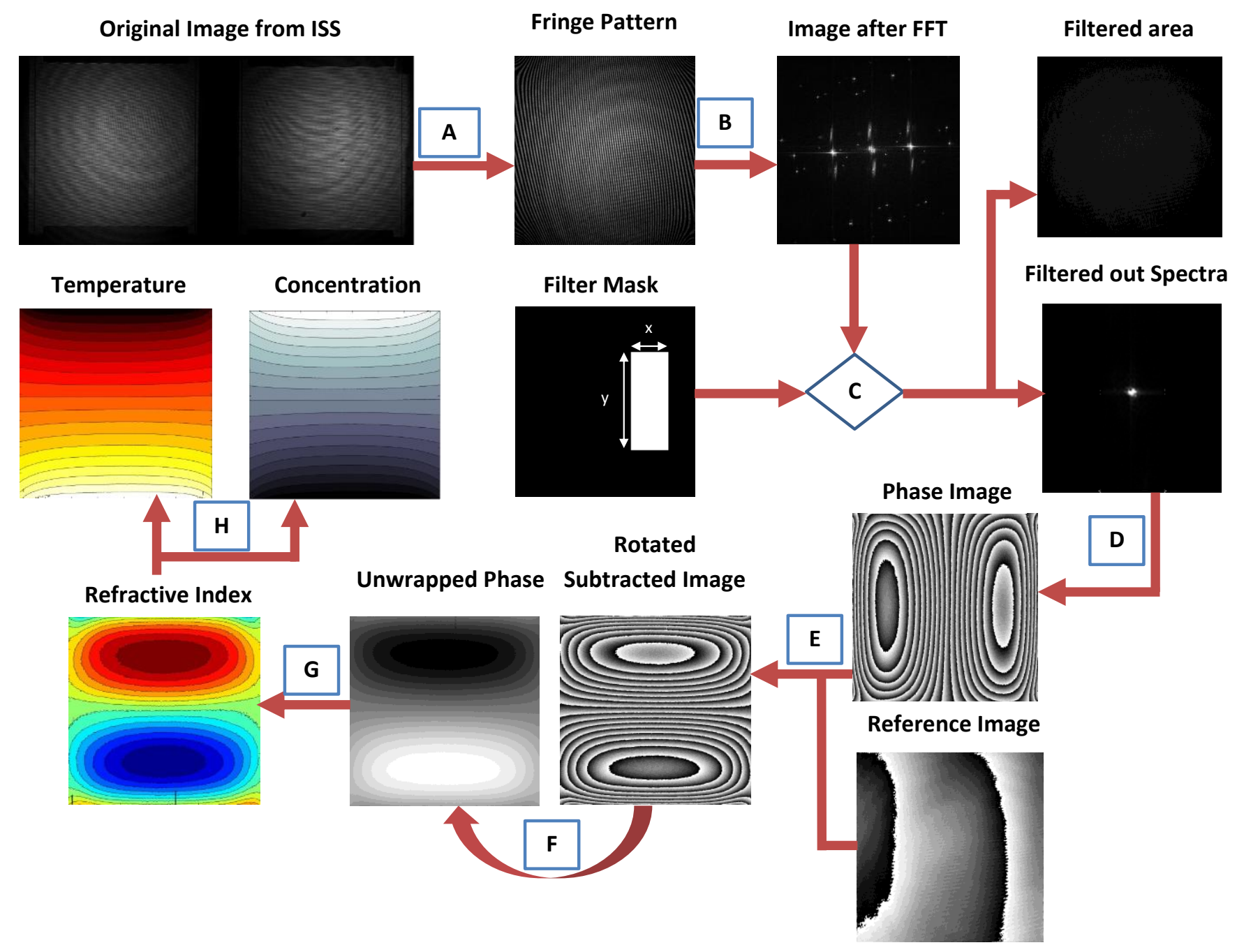

Figure 3-1: Principle scheme of image processing steps (A: Crop the original image to remove wall effect, B: 2D Fast Fourier Transform (FFT), C: Filter out one of the non-zero peaks and bring it in the middle using adjustable [x,y] filter mask, D: Inverse 2D FFT, E: Subtracting the image from reference image, F: Unwrapping the subtracted image, G: Contour of refractive index, H: Contour of Temperature and Concentration) 


\subsection{Calculation of Change in Refractive Index}

The phase difference that is obtained by unwrapping process is used to calculate, $\Delta n$, the total variation in refractive index between cold side and hot side. The relation between phase difference, $\Delta \phi$, and $\Delta n$ is illustrated below in equation 3-14.

$$
\Delta n(x, y)=n(x, y)-n\left(x_{0}, y_{0}\right)=\frac{\lambda}{2 \pi L} \Delta \phi(x, y)
$$

Where, $\mathrm{L}$ is the optical path, which is described as the distance or the depth of the liquid that laser passes through. As it could be seen from the above equation, variation of refractive index is equivalent to the change of the optical phase. Also, $\lambda=670 \mathrm{~nm}$ is the wavelength of $\mathrm{He}-\mathrm{Ne}$ laser concentration and temperature contributed to the variation of refractive index for any given wavelength. This relationship is given in the following formula:

$$
\Delta n(x, y)=\left(\frac{\partial n}{\partial T}\right)_{T_{0}, C_{0}, \lambda} \Delta T(x, y)+\left(\frac{\partial n}{\partial C}\right)_{T_{0}, C_{0}, \lambda} \Delta C(x, y)
$$

Where, $\Delta T(x, y)$ and $\Delta C(x, y)$ are the temperature and concentration changes at the

point $(x, y) .\left(\frac{\partial n}{\partial T}\right)$ is variation of refractive index due to temperature and $\left(\frac{\partial n}{\partial C}\right)$ is variation of refractive index due to concentration.

Optical Digital Interferometry require precise knowledge of $\left(\frac{\partial n}{\partial T}\right)$ and $\left(\frac{\partial n}{\partial C}\right)$. These values are also called contrast factors and their accuracy directly determines the accuracy of the Soret coefficients [53]. These values can be measured using Interferometry technique and calculated for different temperatures. Although direct measurement of contrast factors could be done in Ryerson University Microgravity laboratory, it is outside of scope of this study. Thus, these refractive index variations are taken from different sources in literature [52]. Table 3-1 shows a summary of measured values for contrast factors for three different temperatures of $288 \mathrm{~K}, 298 \mathrm{~K}$, and $308 \mathrm{~K}$. The measured values are obtained by the interferometry technique in a full concentration range for wavelength of $\lambda=670 \mathrm{~nm}$ [53]. 


\begin{tabular}{lccccccc}
\hline \hline$c$, water & \multicolumn{2}{c}{$(\partial n / \partial T)_{p, c} / 10^{-4} \mathrm{~K}^{-1}$} & & \multicolumn{3}{c}{$(\partial n / \partial c)_{p, T} / 10^{-2}$} \\
\cline { 2 - 4 } \cline { 7 - 8 }$T=$ & $288 \mathrm{~K}$ & $298 \mathrm{~K}$ & $308 \mathrm{~K}$ & & $288 \mathrm{~K}$ & $298 \mathrm{~K}$ & $308 \mathrm{~K}$ \\
\hline 0.000 & -4.141 & -4.266 & -4.420 & & & \\
0.052 & -4.243 & -4.369 & -4.505 & & 0.260 & 0.336 & 0.399 \\
0.100 & -4.185 & -4.324 & & -0.564 & -0.517 & \\
0.150 & -4.125 & -4.241 & -4.372 & -1.14 & -1.02 & -0.746 \\
0.200 & & -4.138 & & & -1.45 & \\
0.250 & -3.919 & -4.033 & -4.156 & -2.09 & -1.86 & -1.67 \\
0.300 & & -3.921 & -4.040 & & -2.25 & -2.01 \\
0.351 & -3.705 & -3.809 & -3.922 & -2.83 & -2.62 & -2.42 \\
0.409 & -3.570 & -3.681 & -3.784 & -3.26 & -3.01 & -2.79 \\
0.451 & & -3.585 & & & -3.30 & \\
0.500 & -3.398 & -3.474 & -3.560 & -3.89 & -3.64 & -3.47 \\
0.547 & & -3.359 & & & -4.02 & \\
0.600 & -3.155 & -3.217 & -3.294 & -4.84 & -4.56 & -4.38 \\
0.650 & & -3.062 & & & -5.22 & \\
0.700 & -2.782 & -2.854 & -2.943 & -6.42 & -6.04 & -5.35 \\
0.747 & & -2.574 & & & -6.97 & \\
0.800 & -1.863 & -2.127 & -2.347 & -9.15 & -8.18 & -7.30 \\
0.854 & -1.389 & -1.653 & -1.974 & -9.84 & -8.99 & -8.13 \\
0.899 & -0.9801 & -1.343 & -1.652 & -9.92 & -9.23 & -8.72 \\
0.926 & & & -1.540 & & -9.14 & \\
0.950 & -0.7687 & -1.135 & -1.439 & -9.20 & -8.91 & -8.43 \\
0.975 & & & & & -8.49 & \\
1.000 & -0.7200 & -1.060 & -1.350 & & -7.89 & \\
\hline \hline & & & & & & & \\
\hline
\end{tabular}

Table 3-1: Variation of refractive index with temperature, and concentration, for Water-IPA [53]

Figure 3-2 is taken from another source in literature that shows graphs of $\left(\frac{\partial n}{\partial T}\right)$ and $\left(\frac{\partial n}{\partial C}\right)$ as function of mixture composition at the temperature $T \approx 293 \mathrm{~K}$. Figure 3-2a shows $\left(\frac{\partial n}{\partial c}\right)$ changes sign when the mass fraction of water is about 0.08 . On the other hand, Figure $3-2 b$ shows the temperature dependence $\left(\frac{\partial n}{\partial T}\right)$ that is growing function of water content being negative on whole range and the order of magnitude is about $10^{-4} \mathrm{~K}^{-1}[52]$. The values of refractive index variations are assumed constant for each specific mixture. 

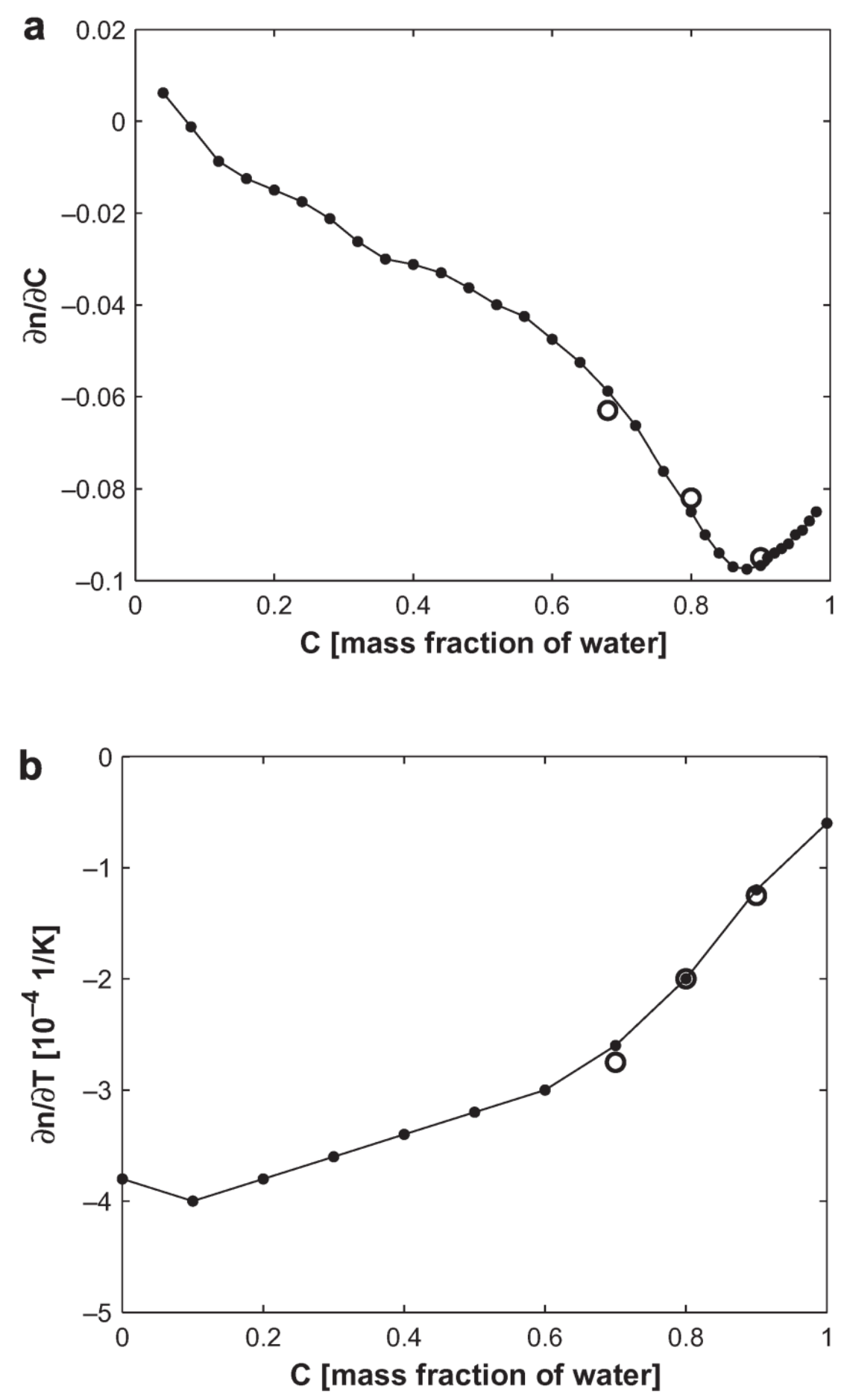

Figure 3-2: Variation of refractive index as function of mass fraction of water. Open circles correspond to laboratory measurements [52] 
As it can be seen from equation 3-15, change in refractive index depends on both concentration and temperature changes in the cavity as a result of applied thermal gradient. For the images recorded early in time of the experiment (i.e. 5-10 $\min ) \Delta T(x, y)=\Delta T_{\text {ref }}(x, y)$. This is because separation is only due to variation of temperature field before the thermal time. In other words, equation 3-16 will be used for the early recorded images instead of equation 3-15.

$\Delta n(x, y)=\left(\frac{\partial n}{\partial T}\right)_{T_{0}, C_{0}, \lambda} \Delta T_{r e f}(x, y)$

However, both temperature and concentration field are responsible for separation of components during thermodiffusion stage and both components of equation 3-15 are used. Then later in time the refractive index will change only due to the Soret induced variations of concentration field. This is in the second stage during diffusion period.

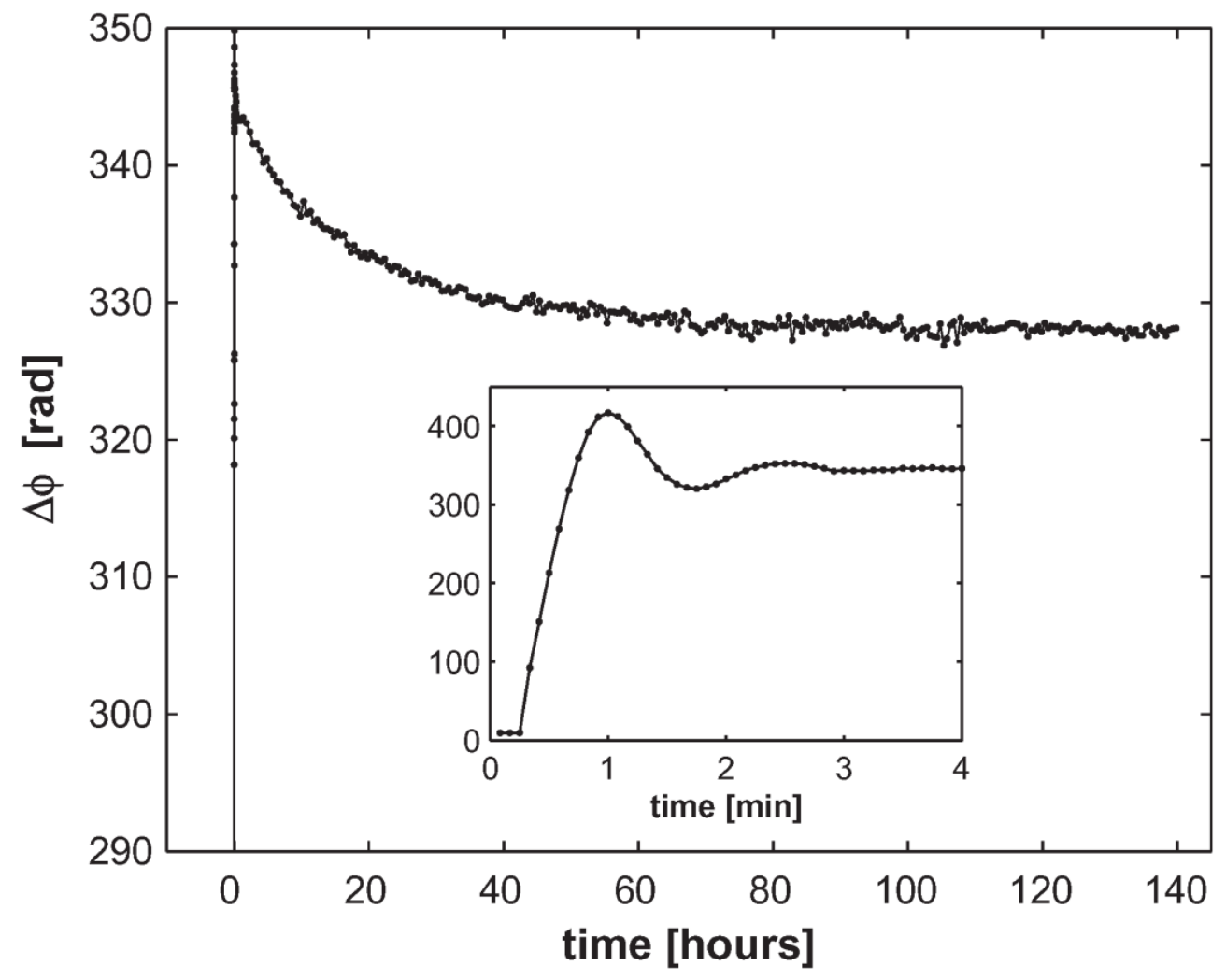

Figure 3-3: Evolution of the phase difference with time during thermodiffusion experiment. Insertion demonstrates the phase change on short time scale when all changes are only due to the establishment of temperature gradient; water/isopropanol mixture with $\mathrm{C}_{0}=0.5$ [52] 
The evolution of the phase difference with time is shown in Figure 3-3 for mixture of (50\% IPA-50\% Water). The main graph presents the progress on long time scale and insertion displays the process on short time scale [52].

The evolution of concentration in space and time is given by equation 3-17:

$C(t, z)=C_{0}+\Delta C_{s t}\left[\frac{z}{L}-\frac{1}{2}+\frac{4}{\pi^{2}} \sum_{n, \text { odd }}^{\infty} \frac{1}{n^{2}} \exp \left(-n^{2} \frac{t}{\tau_{D}}\right) \cos \left(\frac{n \pi z}{L}\right)\right]$

Where $C_{0}$ is the initial concentration, $\tau_{D}=\frac{L^{2}}{\pi^{2} D}$ is the diffusion time, $D$ is the diffusion coefficient, $L$ is the optical length, and $\Delta C_{s t}=-S_{T} C_{0}\left(1-C_{0}\right) \Delta T$ is the steady state separation, where $S_{T}$ is the Soret coefficient. The sign between the first two terms in equation 15 depends on the choice of the component to be analyzed. The sign is negative in the case of heavier component and positive in the case of a lighter component [51]. Thus, the evolution of concentration difference between top and bottom of the experimental cell is defined by equation 3-18:

$\Delta C(t)=C(t, L)-C(t, 0)=\Delta C_{s t}\left[1-\frac{8}{\pi^{2}} \sum_{n, o d d}^{\infty} \frac{1}{n^{2}} \exp \left(-n^{2} \frac{t}{\tau_{D}}\right)\right]$

Nevertheless, steady state condition is assumed to be reached for the purpose of this study. In other words, Soret coefficient, $S_{T}$, is calculated using the steady state separation formula. 


\section{CHAPTER 4 EXPERIMENTAL RESULTS}

In this chapter selected experimental runs according to Table 2-2 are analysed and compared to benchmark values to clarify the role of forced vibration and show the temperature and concentration distribution in the experimental cavity. This is done for both the mixture of (10\% IPA-90\% Water) and (50\%IPA-50\% Water). The latter goes through further discussion as this mixture is unstable especially as higher thermal gradient is applied in the cavity. The mixture of (10\% IPA-90\% Water) is purely aimed to investigate the influence of force vibration on separation ratio.

\subsection{Analysis of Mixture with Negative Soret Coefficient}

In this section, several parameters are taken in to account. $\Delta T$ is kept at constant value of $5 \mathrm{~K}$ for all the four runs. However, frequency and amplitude of applied vibration vary in each run. The main goal is to check any possible effect of vibration on measurement of Soret coefficient. For this purpose, behaviour of water-isopropanol mixture is studied under different Rayleigh vibration. Obtained results are validated by comparing them with the results of the second run that involves no vibration.

\subsubsection{Concentration Profile}

Figure 4-1 shows concentration profile of the mixture during different time of the experiment. Moreover, Table 4-1 indicates values of $\Delta \mathrm{C}$ and $S_{T}$ for each Run at steady state condition after passing 12 hours. It can be observed in Figure 4-1 that the highest concentration difference, $\Delta C$, (i.e. Soret separation) corresponds to Run 2 without any vibration. While, concentration difference between the cold and hot wall is relatively small at higher Rayleigh vibration. In other words, less separation occurs in the mixture as Rayleigh vibration number increase in every run. The reason for such behaviour is that more disturbances are created in the system due to higher Rayleigh vibration. 


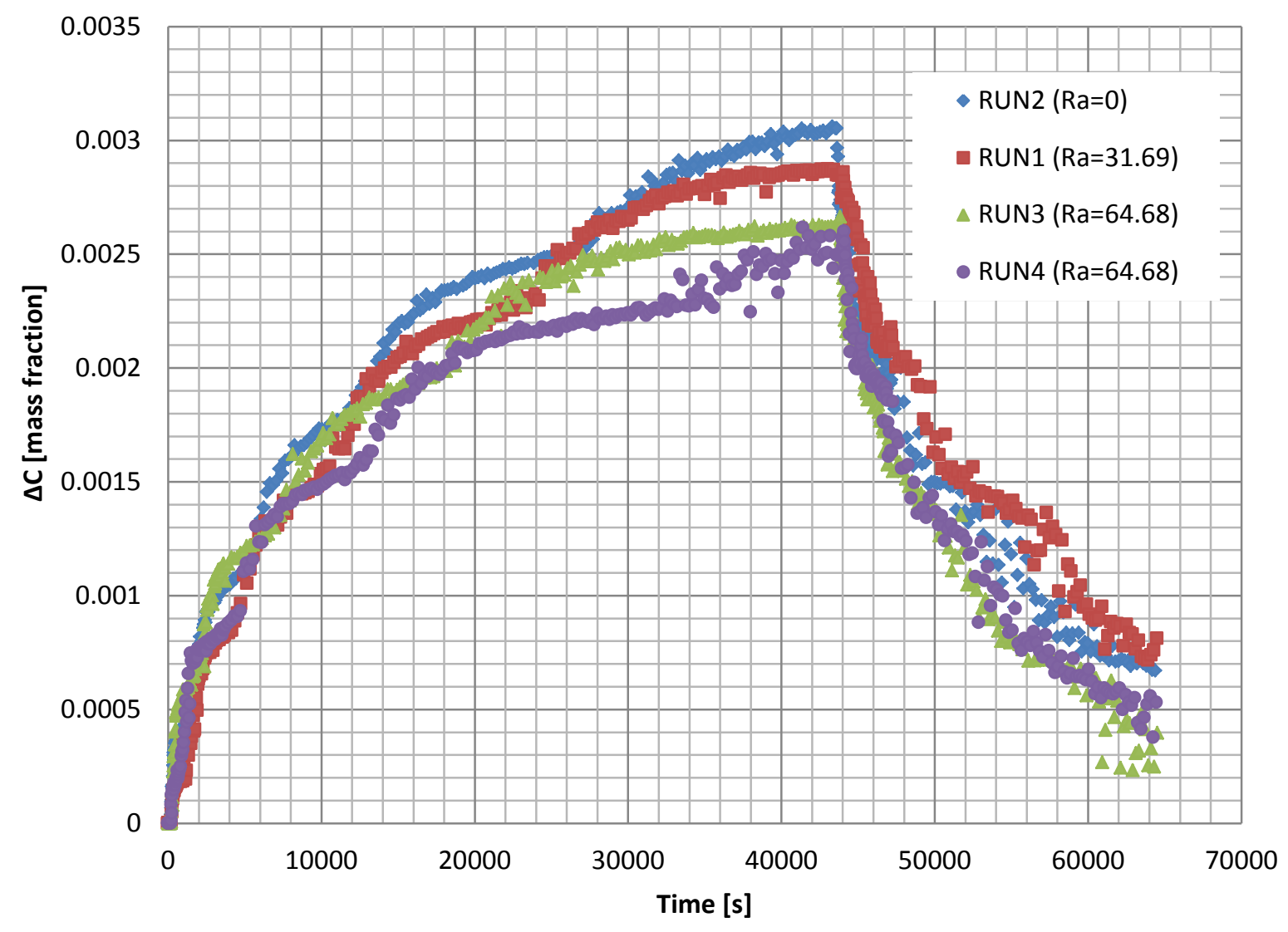

Figure 4-1: Concentration profile at different time for low Rayleigh vibration runs. (Non-uniform sampling rate is due to limited number of downloaded images)

\begin{tabular}{|c|c|c|c|c|c|c|c|}
\hline $\mathbf{R U N} \#$ & $\mathbf{f}[\mathbf{H z}]$ & $\mathbf{A}[\mathbf{m}]$ & $\begin{array}{c}\Delta \mathbf{T} \\
(\mathbf{2 5} \pm \mathbf{\Delta T} / \mathbf{2})\end{array}$ & $\mathbf{R a}_{\text {vibration }}$ & $\Delta \mathbf{C}_{\text {steady }}$ & $\mathbf{S}_{\mathbf{T}, \text { steady }}[\mathbf{1} / \mathbf{K}]$ & $\begin{array}{c}\text { Literature } \\
(\text { V.Shevtsov et al., } \\
\mathbf{2 0 1 0})\end{array}$ \\
\hline $\mathbf{1}$ & 0.5 & 0.070 & 5 & 31.69 & $2.85 \times 10^{-3}$ & $-6.33 \times 10^{-3}$ & \\
\hline $\mathbf{2}$ & 0 & 0 & 5 & 0 & $3.05 \times 10^{-3}$ & $-6.78 \times 10^{-3}$ & $(-6.77 \pm 0.11) \times 10^{-3}$ \\
\hline $\mathbf{3}$ & 2.0 & 0.025 & 5 & 64.68 & $2.62 \times 10^{-3}$ & $-5.83 \times 10^{-3}$ & \\
\hline $\mathbf{4}$ & 1.0 & 0.050 & 5 & 64.68 & $2.50 \times 10^{-3}$ & $-5.55 \times 10^{-3}$ & \\
\hline
\end{tabular}

Table 4-1: Comparison of $\Delta C$ and $S_{T}$ for ISS experimental runs at steady state condition 


\subsubsection{Concentration profile in step I}

Following from Figure 4-1, concentration differences for all the runs are increasing in similar trend until temperature gets stabilized (i.e. end of first thermal time). After passing thermal time, both temperature and concentration are responsible for separation of components. In this period (i.e. Thermodiffusion phase), concentration profiles start to follow different path. While the trend for all the runs is relatively similar, the maximum difference is noticeable at the end of thermodiffusion period. This is where the effect of vibration can be clearly shown. The plots indicate that as Rayleigh number increases concentration difference decreases.

\subsubsection{Concentration profile in step II}

In our calculation it is assumed that separation in the system reaches an acceptable steady state value at the end of approximately 12 hours. After this period, right after shutting down the temperature gradient, the second thermal time starts and takes about the same time as the first one. Again, it is clearly seen from the plots that concentration difference for all the runs follow a similar path and decrease. Again the fluctuation of concentration profile for each run is noticeable after finishing thermal time where diffusion takes over all separation process in the mixture. However, the trend is more linear compare to the thermodiffusion phase.

\subsubsection{Soret Coefficient, $S_{T}$}

\subsubsection{Run 2 without vibration}

Soret coefficient is determined for each experimental runs based on measured concentration difference. The value of Soret coefficient is obtained using the steady-state formula and is $S_{T}=-6.78 \times 10^{-3} 1 / \mathrm{K}$ assuming separation process has reached a steady value. This value is validated with a recent paper published by V. Shevtsova et al. in 2010. 
They also used ISS data to calculate Soret coefficient at steady state for the same mixture (i.e Water-Isopropanol). As follows from Table 4-1, the obtained value for $S_{\mathrm{T}}$ is in good agreement with the literature value

\subsubsection{Runs with vibration}

The case without any vibration has been going under many analyses by many researchers. In contrast, there are no analyses done so far in the cases that vibration is applied to the system. Nonetheless, in this study, Soret separations for runs with low Rayleigh vibration are also compared with the second run. The difference between Soret coefficients of each run with the original run without any vibration is noticeable. As follows from Table 4-1, when the Rayleigh vibration number starts to increase, Soret coefficient decreases accordingly. Thus, ISS experimental data analyses clearly indicate the effect of vibration on Soret coefficient measurement.

\subsubsection{Frequency and Amplitude of Low Rayleigh Vibration}

Furthermore, impact of amplitude and frequency variation could be seen from run 3 and run 4 separations, which have same Rayleigh vibration numbers. Although these vibrations are small and justification of their corresponding separation plots is difficult, results are good indication of frequency and amplitude impact on separation of components.

\subsubsection{Effect of frequency variation on Soret coefficient measurement}

Results show that concentration difference decreases as frequency of vibration decreases from $2 \mathrm{~Hz}$ to $1 \mathrm{~Hz}$. Thus, less separation is appeared in the system due to Soret effect. This behaviour may indicate a direct relationship between frequency of vibration and separation of components. Correspondingly, Soret coefficient, $\mathrm{S}_{\mathrm{T}}$, decreases from run 3 to run 4 as frequency of vibration decreases. This result agrees with finding of other

researchers [54] about effect of frequency of vibration on thermodiffusion. Increase in 
frequency of transitional vibration to higher values results in appearance of averaged flow that effect heat and mass transport in a system [54]. This would increase the separation of components in the mixture resulting in a higher Soret coefficient.

\subsubsection{Effect of amplitude variation on Soret coefficient measurement}

Conversely, less separation appears in the mixture as amplitude of vibration increases from run 3 to run 4 . In other words, steady state Soret coefficient, $S_{\mathrm{T}}$, reduces by increasing the amplitude of vibration from $25 \mathrm{~mm}$ to $50 \mathrm{~mm}$. This result illustrates a probable inverse relationship between amplitude of vibration and separation of components.

These findings are clear indication of fluid behaviour under different frequency and amplitude because all other parameters are kept the same in the two runs (3 and 4).

In short, results are in good agreement with benchmark values according to Table 4-1. This means that we can draw reliable conclusion based on our results. From the trends, it is unclear whether vibration would impact the results significantly. There is some nonlinearity in the thermodiffusion part of each plot. Whereas, in the second part (separation occurs due to pure diffusion) more linear concentration change could be seen from the plots. In all the cases, concentration profile somehow is linear because low Rayleigh vibrations are applied to the system. However, the amount of mixing in the cavity due to vibration increases that reduces separation of components due to thermodiffusion.

Results clearly indicate the influence of different vibration levels on thermodiffusion measurement in our test mixture. The vibration causes convection and remixes the fluid mixture resulting in imperfect development of Soret separation. Also, having a non-uniform temperature can be a source of convection in the system. The calculated Soret coefficient from Image Processing is in good agreement with the benchmark value. Nonetheless, the effect of vibrations is clearer during thermodiffusion time where separation is due to Soret effect. Results specify a possible relationship between separation and frequency, and between separation and amplitude of the vibration. Also, based on the results less 
separation appears within isopropanol and water components as Rayleigh vibration number increases. Results show maximum separation in the case without any vibration.

\subsubsection{Temperature Distribution in the Experimental Cavity}

Figure 4-2, Figure 4-3, and Figure 4-4 show temperature distribution in the cavity during thermal time for runs 1, 2, and 3 respectively. The pattern of temperature distribution in the cavity is almost similar for all the runs regardless of the magnitude of their vibration. The first image shows the moment right before the temperature is applied to the system. Linearization process of temperature is shown in the second, third image that are taken 20 second and 40 second after applying temperature respectively. Consequently, the last image is taken 770 second after applying temperature. According to the given relation for thermal time stated in Chapter 2, this parameter for water/IPA mixture is close to 770 s. Linear variation of thermal gradient is reached in the cavity at the end of thermal time. The separation of components begins as soon as the thermal gradient is applied. Nevertheless, this separation can be neglected because separation is a slow process; thus neglecting few minutes of this process may not cause a noticeable influence. Although this assumption does not count as a source of visible change in the final result, it is vital to consider the separation as soon as linear temperature is achieved between two walls as result of high ratio of separation at beginning. Clearly, similar contours for all three cases are resulted regarding different times. In addition to this similarity, the approximate linear variation between cold and hot sides can also be perceived. Lateral temperature gradient can be noticeable in the cavity. The curvature pattern at four corners of the cavity at the end of thermal time may be due to technical design of the cell and as a result of imperfect experimental setups. Although IVIDIL experiment is done in a complete weightless condition in microgravity environment, an ideal condition can never be achieved in any experiment. An environment with no convection is defined as an ideal case for this experiment. However, temperature field in IVIDIL approaches the corner regions in a nonlinear pattern, meaning existence of convection in the cavity. This would directly influence 
the separation process and create mixing in the cavity. In other words inaccurate thermal design of the experimental cell would defeat the purpose of carrying the experiment in a microgravity environment.

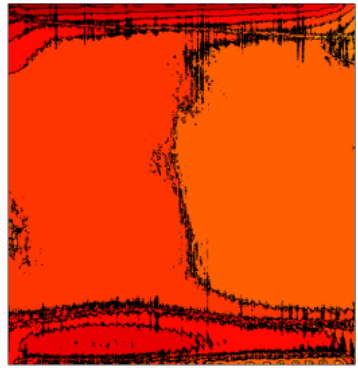

$\mathbf{t}=\mathbf{0}[\mathrm{sec}]$

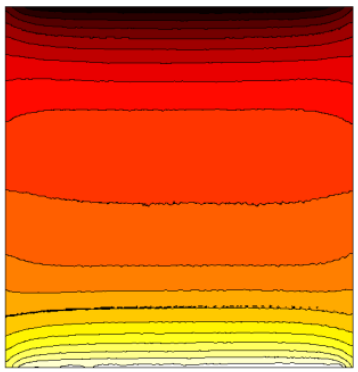

$\mathbf{t}=27[\mathrm{sec}]$

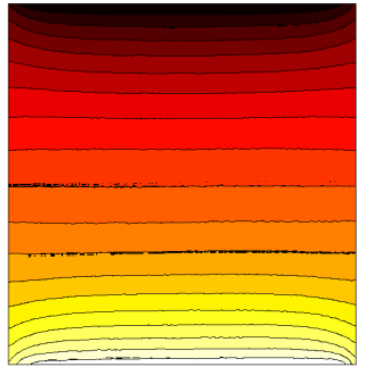

$\mathrm{t}=47[\mathrm{sec}]$

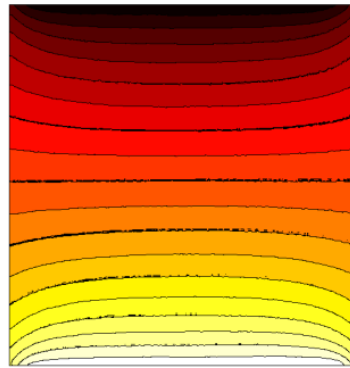

$\mathbf{t}=770[\mathrm{sec}]$

Figure 4-2: Temperature distribution in the cavity during thermal time. Run 1

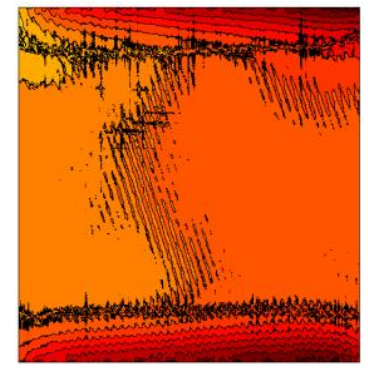

$\mathbf{t}=\mathbf{0}[\mathrm{sec}]$

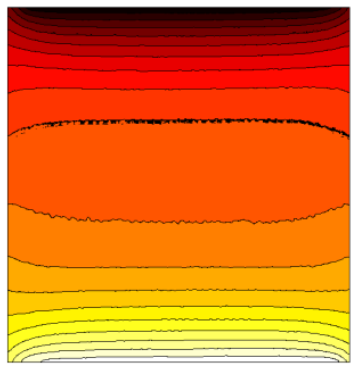

$\mathbf{t}=\mathbf{2 7}[\mathrm{sec}]$

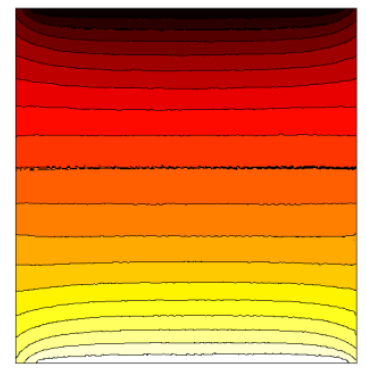

$\mathrm{t}=47[\mathrm{sec}]$

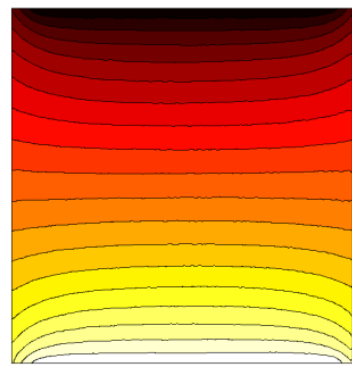

$\mathrm{t}=770[\mathrm{sec}]$

Figure 4-3: Temperature distribution in the cavity during thermal time. Run 2

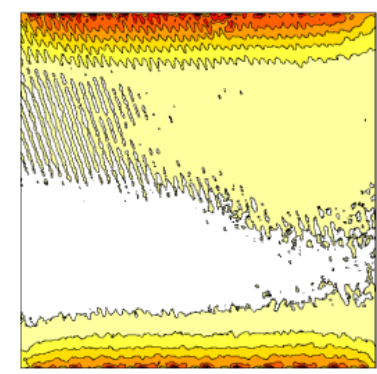

$\mathbf{t}=\mathbf{0}[\mathrm{sec}]$

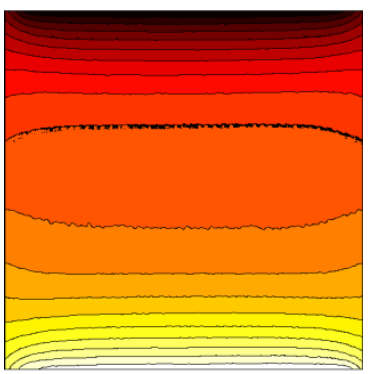

$\mathbf{t}=\mathbf{2 7}[\mathrm{sec}]$

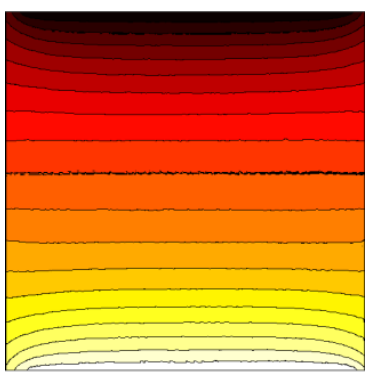

$\mathbf{t}=47[\mathrm{sec}]$

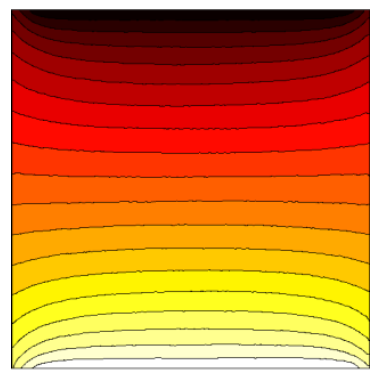

$\mathbf{t}=770[\mathrm{sec}]$

Figure 4-4: Temperature distribution in the cavity during thermal time. Run 3 


\subsubsection{Concentration Distribution in the Experimental Cavity}

Figure 4-5, Figure 4-6, and Figure 4-7 are used to illustrate the distribution of concentration inside the IVIDIL cell in runs 1, 2, and 3 respectively at various times. Noticeably, there are different contours for various cases. Also, it is clearly seen that due to thermodiffusion the concentration field changes near the lateral walls. Though the separation mainly begins close to hot and cold sides, the interesting observation is that the separation starts from two lateral walls as well. Whereas, these walls are meant to provide adiabatic condition. This phenomenon can be seen during first 30 minutes of diffusion time. This is as a result of heat flux which exists close to corners of the cavity due to inappropriate isolation setup. When the separation in the domain becomes more pronounce after about one hour, this observation diminishes. In other words, on long time scale it will diffuse and concentration field becomes similar to temperature field. Concentration contours usually behave like temperature contours. As mentioned, temperature contours shows high temperature gradient close to corners of the cavity because of small gaps at the corners. Thus, these temperature variations affect the concentration pattern in the cavity mostly close to each corner at end of thermodiffusion phase. The similar temperature and concentration patterns between hot and cold walls can be observed clearly in the domain for run 2 with zero Rayleigh number which shows higher separation at the middle of the cavity. While for runs 1 and 3 the similarity between concentration and temperature field cannot be observed. Conversely, As a result of higher level of vibration, higher concentration exists closer to the corners. It demonstrates a strong induced velocity field which is caused by at least one strong convection cell in the cavity due to force vibration. This observation has its maximum effect on run3, in which diffusion pattern is destroyed by two strong concentration cells at the top and bottom of the cavity and create two different regimes with almost homogenize concentration profiles.

In short, Rayleigh vibration can destroy pure separation pattern, while it may not cause a remarkable deviation from linear temperature variation from zero gravity condition. 


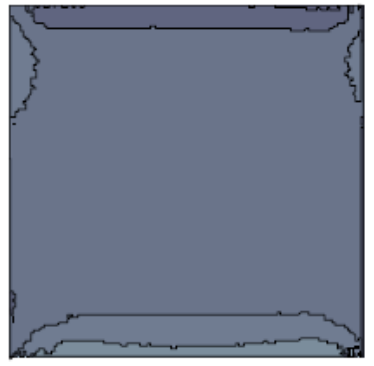

$\mathbf{t}=\mathbf{1 0}[\min ]$

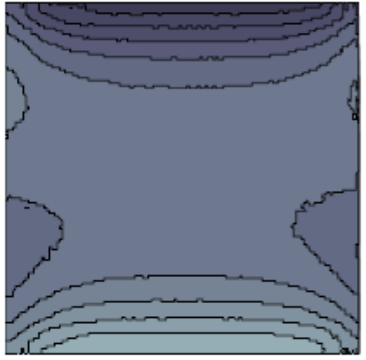

$\mathbf{t}=\mathbf{3 0}[\mathrm{min}]$

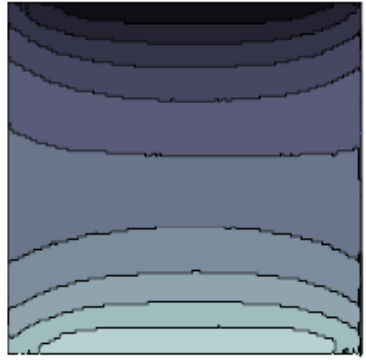

$\mathrm{t}=\mathbf{1}[\mathrm{hr}]$

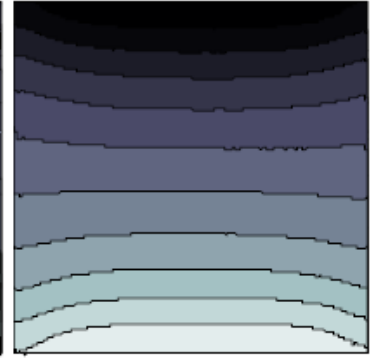

$\mathrm{t}=12[\mathrm{hr}]$

Figure 4-5: IPA concentration profile in the cavity at different time during diffusion time, Run1

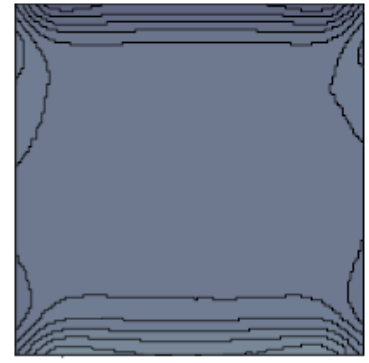

$\mathrm{t}=\mathbf{1 0}[\mathrm{min}]$

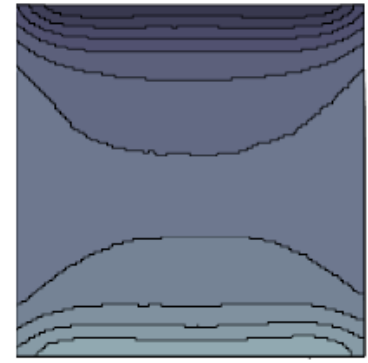

$\mathbf{t}=\mathbf{3 0}[\min ]$

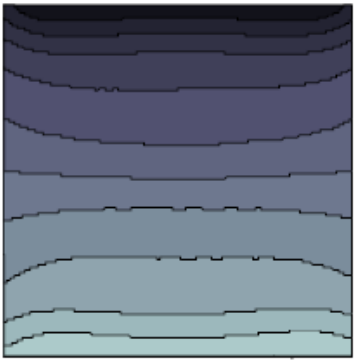

$\mathrm{t}=\mathbf{1}[\mathrm{hr}]$

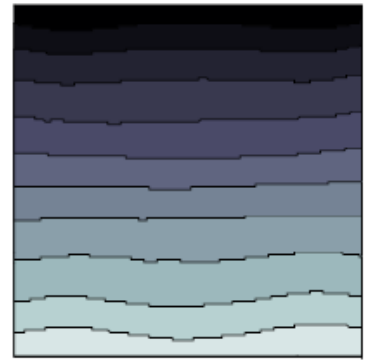

$\mathrm{t}=12[\mathrm{hr}]$

Figure 4-6: IPA concentration profile in the cavity at different time during diffusion time, Run2

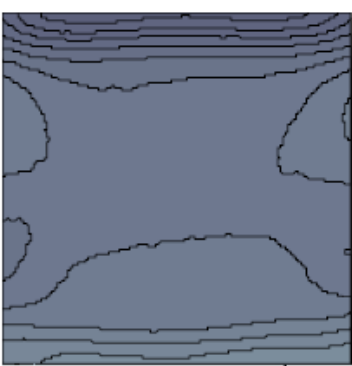

$\mathrm{t}=\mathbf{1 0}[\mathrm{min}]$

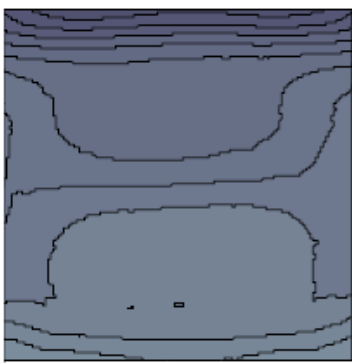

$\mathbf{t}=\mathbf{3 0}[\mathrm{min}]$

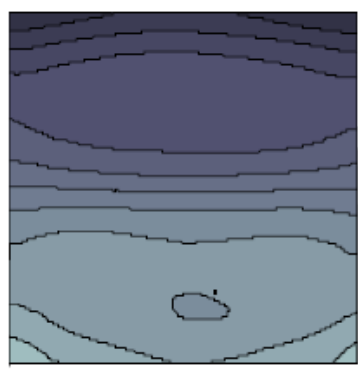

$\mathrm{t}=\mathbf{1}[\mathrm{hr}]$

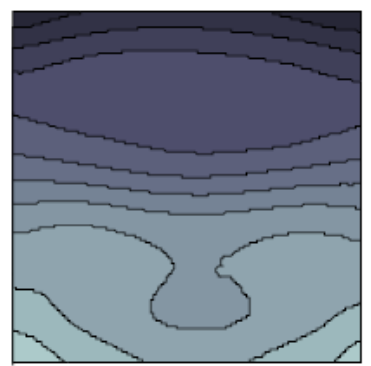

$\mathrm{t}=12[\mathrm{hr}]$

Figure 4-7: IPA concentration profile in the cavity at different time during diffusion time, Run3 


\subsection{Analysis of Mixture with Positive Soret Coefficient}

This section is dedicated to analyse the mixture with positive Soret coefficient which is $50 \%$ IPA and $50 \%$ water. The behaviour of the mixture in the second sets of runs (29 Runs are devoted to 50\% IPA and 50\% water) is noticeably different from the first set and it is not only due to the different Soret sign. This means that other factors such as temperature, thermal and cell design, applied vibration, and experimental setup create chaotic behaviour in the experimental mixture during thermodiffusion measurements.

The basic fluid mechanics indicates that lighter fluid rises while heavy fluid sinks in gravity field. However, a phenomenon such as isothermal Rayleigh-Taylor instability oppose this fact and forces the heavy fluid be placed on top of a light one [55]. In the case of negative Soret effect, unstable density stratification might be established in a binary mixture when heating from above. In other word, the heavier liquid accumulates on top of the lighter one. The system faces buoyant instabilities due to the large differences between viscous, thermal and diffusion times [55].

Similarly, as IVIDIL reports call it "unknown kind of instability" that is an unstable behaviour of mixture occurs during the experiment while testing the mixture with positive Soret coefficient. Furthermore, positive Soret coefficient implies that temperature difference drive the lighter component of the mixture into the direction of higher temperature. This behaviour indicates that Soret effect enhances the buoyancy forces that result from the temperature dependence of the density [56]. In other words, in a binary mixture, the lighter component with positive separation ratio migrates to the hot wall of the experimental cavity, which augments thermal buoyancy enhancing instability in the system.

In general, there is insufficient amount of research and experimental information regarding analysis of thermodiffusion in mixtures with positive separation ratio. This issue

creates difficulty in making quantitative or even qualitative measurements and drawing final conclusions when Soret coefficient is positive. 


\subsubsection{Analysis of Run 34 Results}

The condition of Run 34 in terms of vibration and temperature is as follow: This run has frequency of $0.5 \mathrm{~Hz}$ and amplitude of $0.07 \mathrm{~m}$ which gives Rayleigh number of 100.90 based on equation 2-1. The temperature difference between the top and bottom plate of the cell is $(\Delta \mathrm{T}=5 \mathrm{~K})$ which is assumed to be uniformly distributed in the experimental cavity. Figure 4-8 shows the concentration profile in the direction of temperature gradient versus the duration time of the experiment.

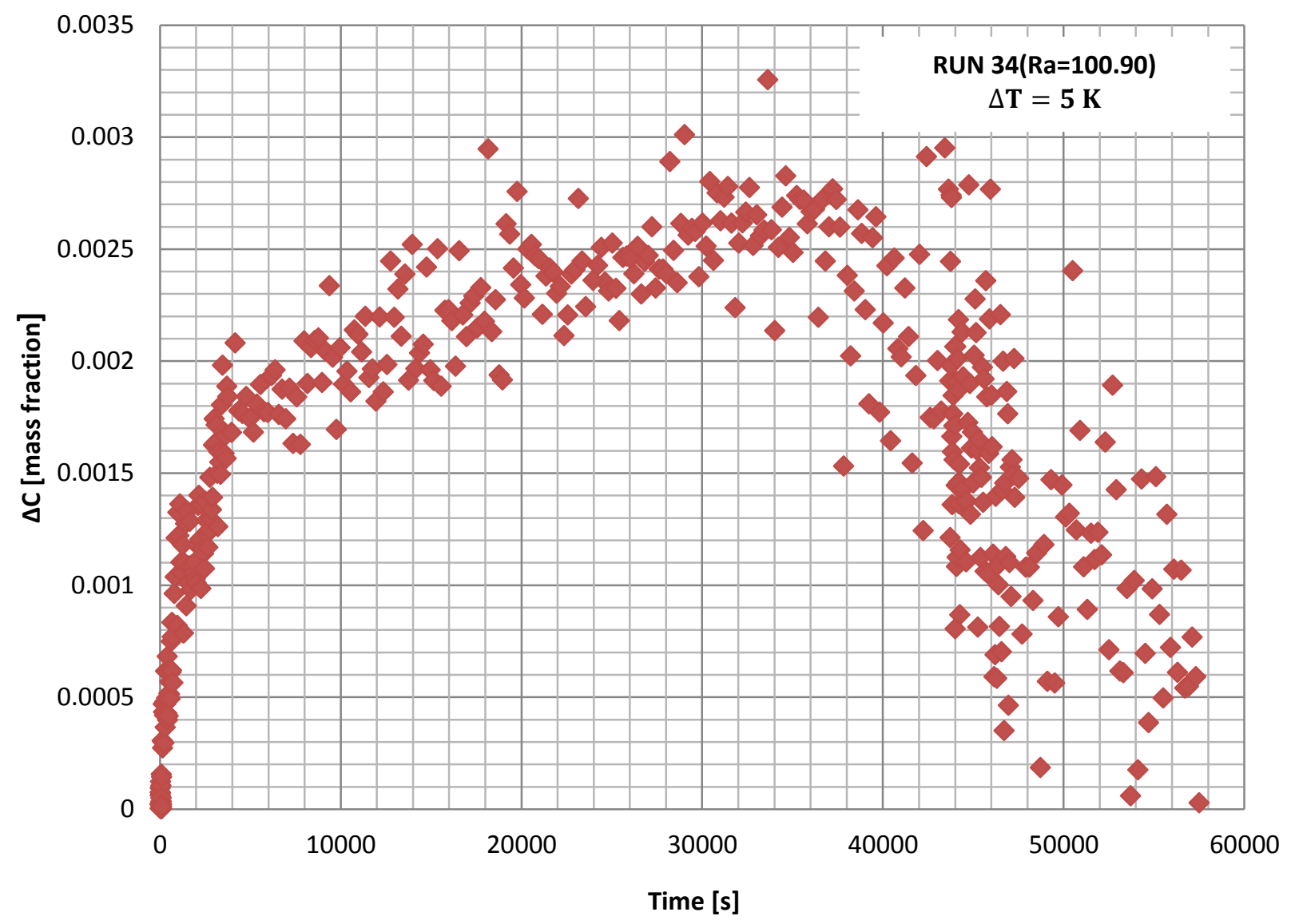

Figure 4-8: Concentration profile for run 34

As it can be seen from the graph, the trend starts with a fast increase. This indicates that separation begins as soon as the temperature is applied to the system. After the temperature is stabilized in the system, separation process starts to follow a slower pace until the 12 hours of applied temperature is completed. Scattered data during both the thermodiffusion 
and diffusion steps could be resulted from various reasons such as the instability of the system, lack of number of available raw images, and possibility of incompatible processing technique. However, the trend of concentration is reasonable considering the above possible issues. Nonetheless, $\Delta \mathrm{C}$ profile is assumed to reach a steady state at the end of 12 hours and slow diffusion process starts to take over separation right after temperature is stopped. Please note that maximum concentration difference based on the available data is $2.79 \times 10^{-3}$ which in turn gives $S_{T}=2.23 \times 10^{-3} 1 / K$ (refer to Table 4-2).

Figure 4-9 shows fringe pattern and refractive index of image number 52 chosen right after temperature is stabilized in the cavity. In other words, the image is taken after the thermal time around 13 minutes after applying the temperature. A linear fringe pattern is seen in the cavity as $\Delta \mathrm{T}=5 \mathrm{~K}$ that is considered to be small. This means less mixing exists in the cavity and any mixing or disturbance is mostly due to induced vibration.
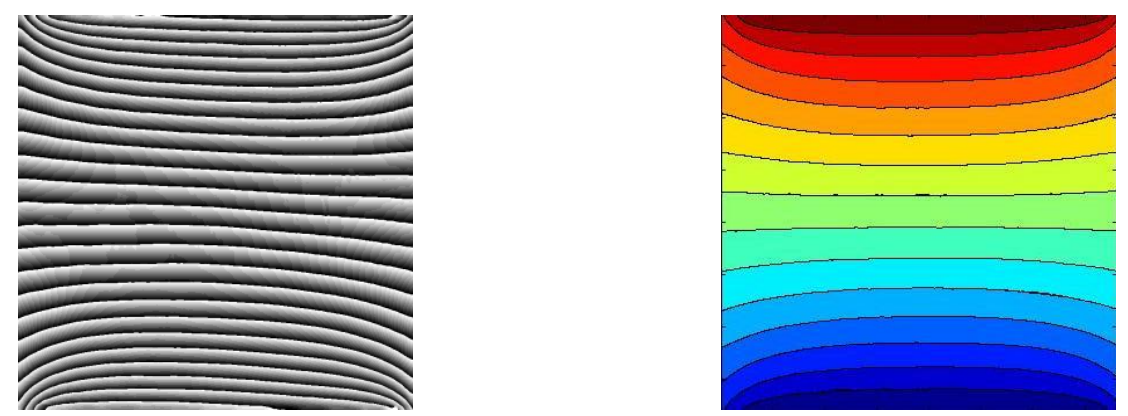

Figure 4-9: Fringe pattern and refractive index, run 34

\subsubsection{Temperature Distribution}

Figure 4-10 illustrates temperature distribution inside IVIDIL cell during the thermal time at different instances of time. The thermodiffusion process starts not only near the hot and cold walls but also near lateral walls because of an additional lateral temperature gradient [45]. This type of behaviour is noticeable for all the runs regardless of the magnitude of their vibration. Linear temperature pattern is seen in the cell at the end of thermal time with slight curvature in all the four corners of cavity. This means convection still exists in the system as a result of vibration and thermal design of the cell. 


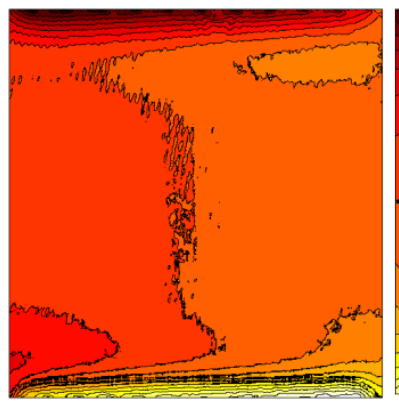

$\mathrm{t}=0[\mathrm{sec}]$

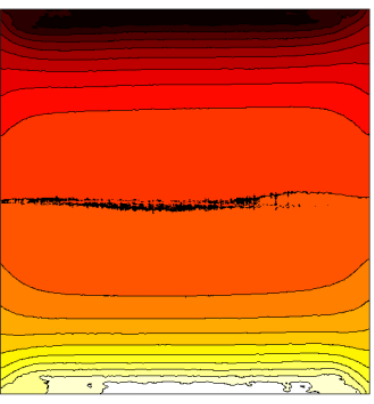

$\mathbf{t}=\mathbf{2 7}[\mathrm{sec}]$

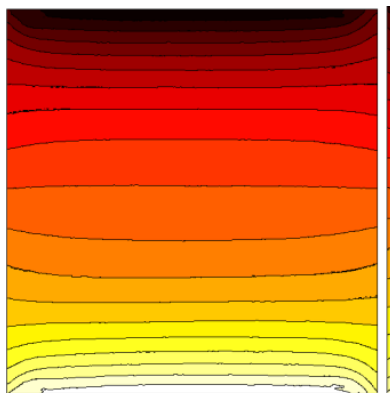

$\mathrm{t}=47[\mathrm{sec}]$

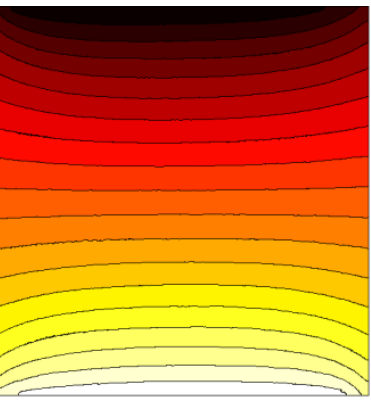

$\mathrm{t}=\mathbf{7 7 0}[\mathrm{sec}]$

Figure 4-10: Temperature distribution in the cavity during thermal time. Run 34

It has been stated that thermal time for water/IPA mixture is close to 770s. This parameter is based on the given relation for thermal time stated in chapter 3. Consequently changes of refractive index in this phase occur due to temperature change in the cell. Liquid properties and contract factor at mean temperature of $298 \mathrm{~K}$ which can be found in Table 2-1and Table 3-1 are used to measure the temperature field in the cavity at each pixel of image with a small error bar with maximum of $0.1 \mathrm{~K}$. The separation of components begins as soon as the thermal gradient is applied. Nevertheless, this separation can be neglected because separation is a slow process; thus neglecting few minutes of this process may not cause a noticeable influence.

The importance of cell design along with thermal design has been mentioned in the previous sections. Moreover, the effect of thermal design of the cell on temperature field has been emphasized on the latest IVIDIL technical report from ISS [27]. It has been shown that existence of additional lateral temperature gradient may be due to technical design of the cell and as a result of imperfect experimental setups [45]. The small thin gap between the quart glasses and both cold and hot wall of the cell-setup causes non-linearity starting from corners of the cavity. From the internal side of the O-rings, the small gap between copper and quartz is filled by working liquid. On the other hand, from external side the gap is filled by surrounding gas that provides particularly inappropriate heat conductor [46]. Consequently, the largest thermal gradient arises at the corner mainly due to the improper 
heat conductivity of the rubber sealing and region nearby. Nonetheless, as experiments are never ideal, the temperature field in IVIDIL approaches the corner regions in a non-linear pattern, even though this non-ideality does not provoke convection in weightlessness condition [57].

\subsubsection{Concentration Distribution}

In the (50\% IPA-50\% Water) mixture diffusion process is too slow in comparison with the (10\% IPA-90\% Water) mixture. This means that the lateral concentration variations cannot be eliminated in the cavity. The distribution of the concentration in the cell for run 34 is shown at various times in Figure 4-11.

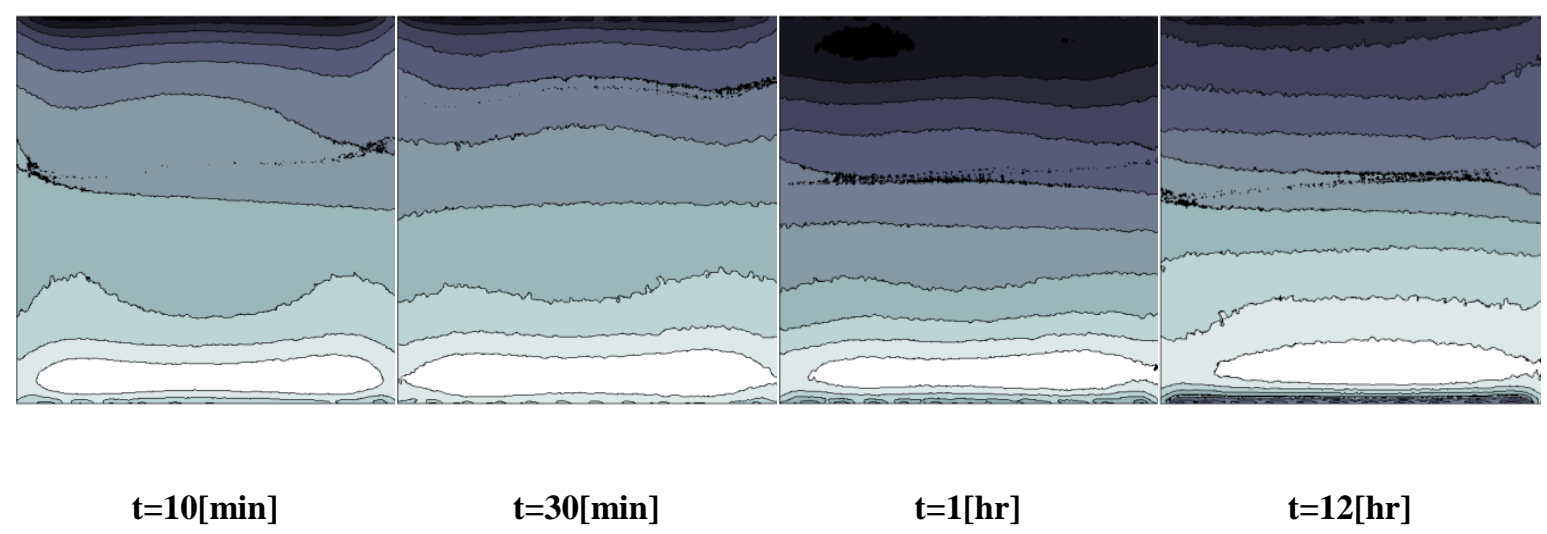

Figure 4-11: IPA concentration profile in the cavity at different time during diffusion time, Run34

Though the separation mainly begins close to hot and cold sides, the interesting observation is that the separation starts from two lateral walls as well. Whereas, these walls are supposed to provide an adiabatic condition. This phenomenon can be seen during first 30 minutes of diffusion time Figure 4-11. This is as a result of heat flux which exists close to corners of the cavity due to inappropriate isolation setup. When the separation in the domain becomes more pronounce after about one hour, this observation diminishes. Concentration contours usually behave like temperature contours. As mentioned in the 
previous section temperature contours shows high temperature gradient close to corners of the cavity because of small gaps at the corners. Thus, these temperature variations affect the concentration pattern in the cavity mostly close to each corner at end of thermodiffusion phase. Furthermore, at $\mathrm{Ra}=100.90$ disturbances are expected in the system which slightly reduce the separation in this case.

\subsubsection{Concentration profile comparison}

In Figure 4-12 concentration profile of Run 34 (50\% IPA-50\% water) is compared with Run 1 (10\% IPA-90\% Water). These two runs have similar condition meaning same frequency and amplitude and temperature difference are applied to the system. However, Rayleigh number varies because physical properties are different. The trend of concentration for Run 1 follows a linear pattern throughout the experiment and steady state condition is reached after the 12 hours. Whereas, the trend for run 34 shows instabilities and fluctuation in the system as a result of higher Rayleigh vibration. Although experimental data are scattered in this profile, the plot shows similar trend as Run 1 (10\% IPA-90\% water) mixture. After applying temperature, separation follows gradual path in both runs until temperature stabilizes in the system. On the other hand, different numbers of image are provided for each runs. The numbers of images in run 34 are less than the number of images in run 1. For instance, the total number of raw images sent from ISS for run 34 is only 350 whereas for run 1 is 700 images.

Accordingly, experimental data are closer together in run 1 which provides more accurate results. In other words, the gap between each image is larger in run 34 considering the duration of the experiment. Thus, the image processing technique is influenced by not having enough data to be processed. Note that every point in the graph represents an image taken at subsequent instants of the experiment. Nevertheless, the image processing technique provides reasonable results for run 34 even though unstable system is expected in (50\% IPA-50\% water) mixture. 


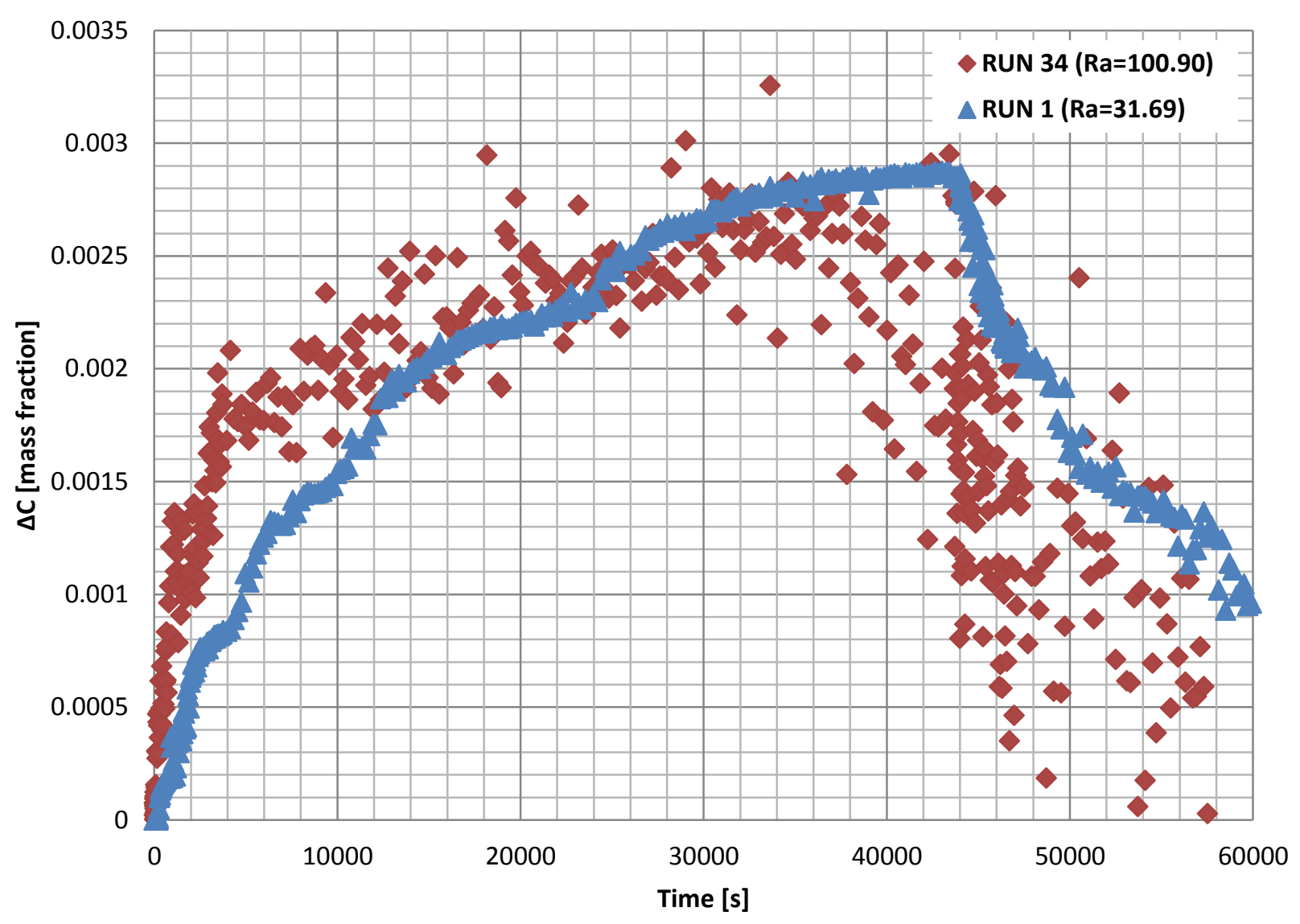

Figure 4-12: Concentration profile for run 34 and run1 comparison

It should be noted that during the first hour the results are following similar pattern but then unknown kind of instability happens which influences the further separation of components. As other groups of researches suggest, performing new runs with higher vibration with homogenous initial conditions can clarify the origin and mechanism of the observed intriguing phenomena [43].

\subsubsection{Analysis of Run 36 Results}

The vibration and temperature that are applied in run 36 are as follow: This run has frequency of $0.5 \mathrm{~Hz}$ and amplitude of $0.07 \mathrm{~m}$. The temperature difference between the top and bottom plate of the cell is $(\Delta \mathrm{T}=10 \mathrm{~K})$ which is assumed to be uniformly distributed in 
the experimental cavity. These properties provide Rayleigh number of 403.60 based on equation 2-1. Figure 4-13 shows the concentration profile in the direction of temperature gradient versus the duration time of the experiment.

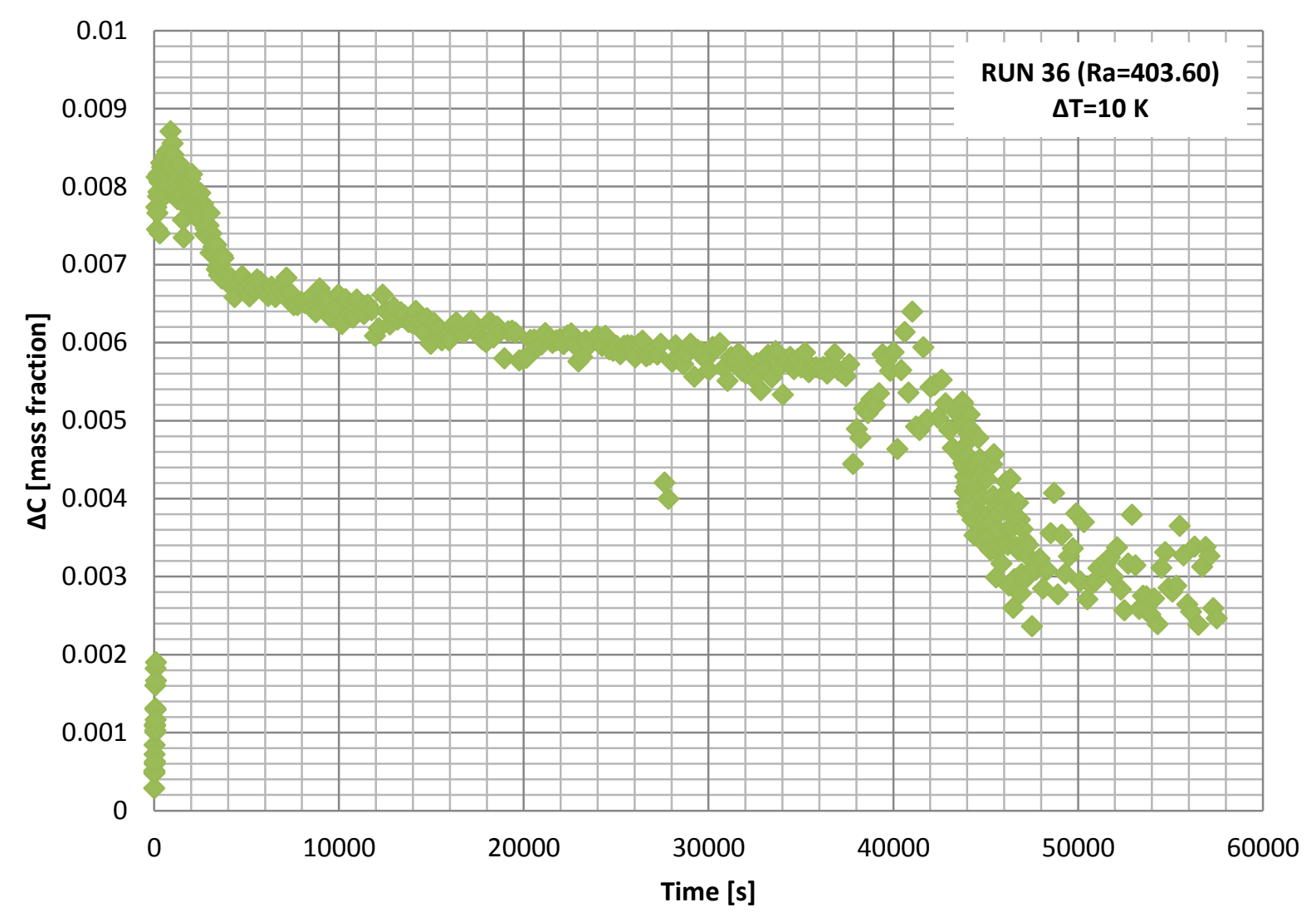

Figure 4-13: Concentration profile for run 36

This run is selected to study the effect of temperature difference while applying force vibration with same amplitude and frequency of run 34. Literature reviews and theoretical studies show that instability of mixture with positive Soret coefficient increases as higher thermal gradient is applied. The concentration profile for this run needs to be analysed as different behaviour is observed in comparison with other runs.

The main issue could rise from the image processing technique used to analyse the data. The method provides a different $\Delta \mathrm{C}$ profile as $\Delta \mathrm{T}$ increases to $10 \mathrm{~K}$. The suitability of the technique can be questioned since it might not be compatible with mixture of (50\% IPA- 
$50 \%$ Water). Images are processed with inbuilt software with no control over changing the process other than cropping the images. However, there is a limit in cropping the images because the applied temperature on top and bottom walls should not be ignored. Moreover, images need to have equal number of pixels in height and width for proper image processing. Having said that Figure 4-14 shows fringe pattern and refractive index of image 25 showing the time when temperature is stabilized in the system.
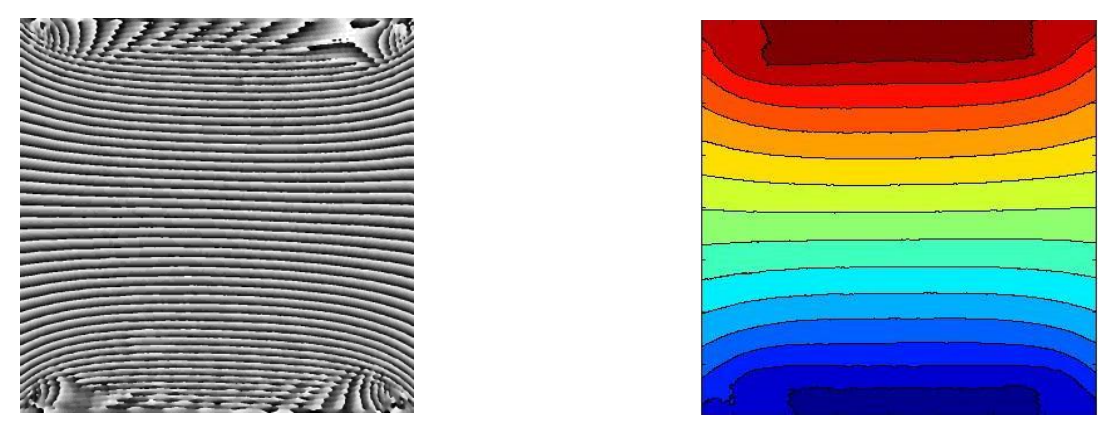

Figure 4-14: Fringe pattern and refractive index, run 36

Fringes are distorted at top and bottom of the cavity. Several reasons could cause this pattern on top and bottom. As temperature difference increases, more convection is observed in the cavity. Also, the small gap between the glass walls and copper plates could result to creating convection in the cavity. Nonetheless, the distortion or noises in fringe pattern cause significant error in unwrapping process and provide less accurate results. This is because every image contributes to prediction of maximum concentration and separation in the mixture. Hence, the sudden jump in $\Delta \mathrm{C}$ profile right after the beginning of separation is not only because of unusual behaviour of the mixture, but also the quality of processed images. Concentration goes down at that point when it is expected to have smooth trend with gradual increase during thermodiffusion process while thermal gradient is still applied to the system. However, the images are close together meaning the trend of stabilization is still the same and lead to reasonable result. Assuming the system has reached a steady state region and using maximum concentration difference of $\Delta C=5.5 \times 10^{-3}$ would give $S_{T}=2.19 \times 10^{-3} 1 / K$ according to Table $4-2$. The effect of vibration is still visible when 
comparing this Run with Run 34.Separation decreases considering higher Rayleigh vibration number. Furthermore, the second stage of concentration profile follows a smooth trend when pure diffusion is slowly taking over separation process.

\subsubsection{Analysis of Run 38 Results}

The vibration and temperature that are applied in run 38 are as follow: This run has frequency of $0.1 \mathrm{~Hz}$ and amplitude of $0.07 \mathrm{~m}$.

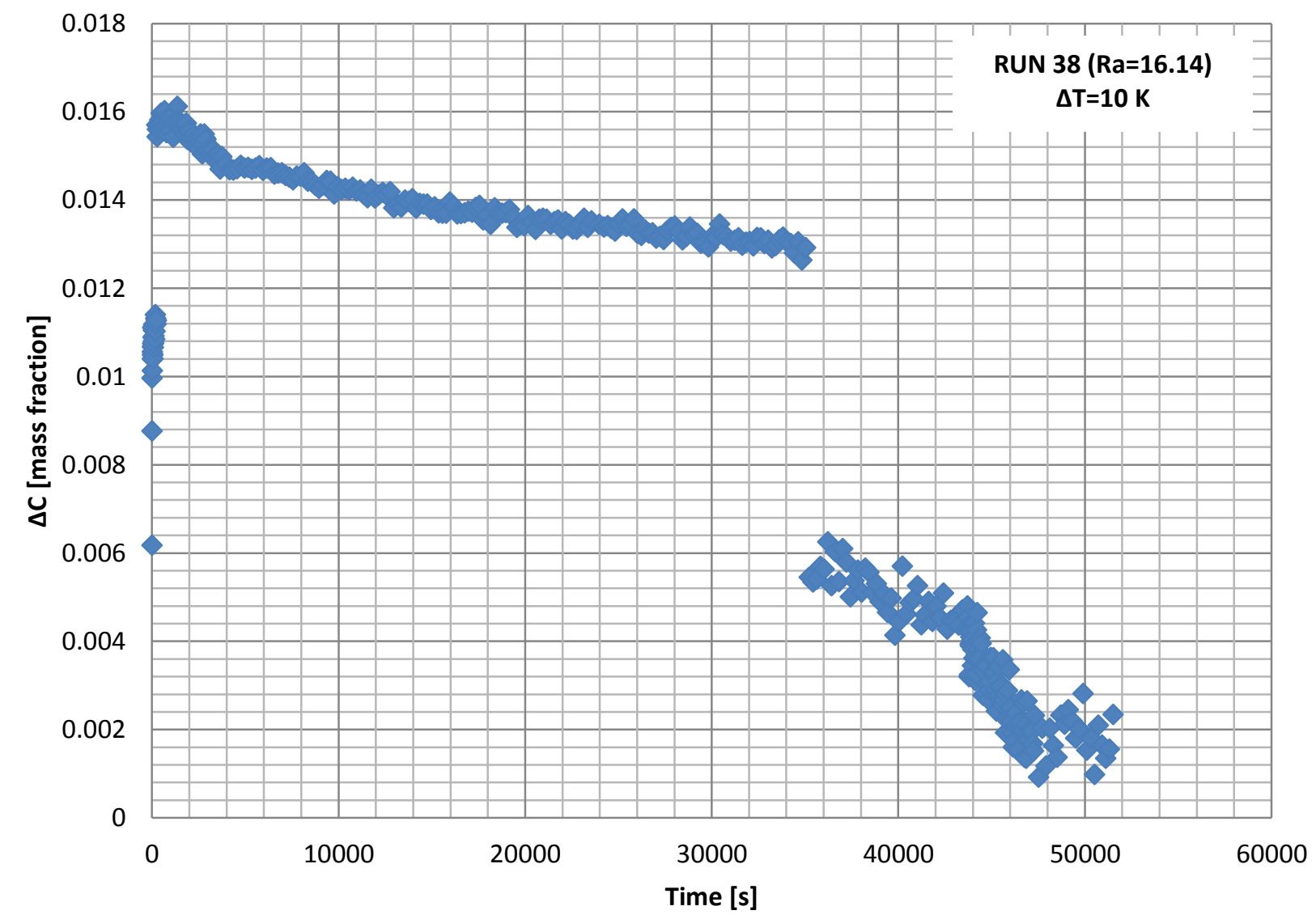

Figure 4-15: Concentration profile for run 38

The temperature difference between the top and bottom plate of the cell is $(\Delta T=10 \mathrm{~K})$ which is assumed to be uniformly distributed in the experimental cavity. These properties provide Rayleigh number of 16.14 based on equation 2-1. Figure 4-15 shows the concentration profile in the direction of temperature gradient versus the duration time of the 
experiment.

This plot shows similar trend of concentration difference as previous runs only the data are closer together as a result of low frequency vibration. Although the applied temperature difference is high, smooth graph is obtained with less fluctuation. Once again, this result might be influenced by the image processing method and lack of provided data from ISS.

Figure 4-16 shows fringe pattern and refractive index of image 52 chosen right after temperature is stabilized in the cavity. In other words, the image is taken after the thermal time around 13 minutes after applying the temperature. Although $\Delta \mathrm{T}=10 \mathrm{~K}$ and some disturbances are expected as per the fringe image, refractive index shows linear pattern as result of low frequency vibration. This means any mixing in the cavity is mostly due to induced vibration. Consequently, refractive index provides a good quality image that can be used for obtaining temperature distribution in the system and concentration profile.
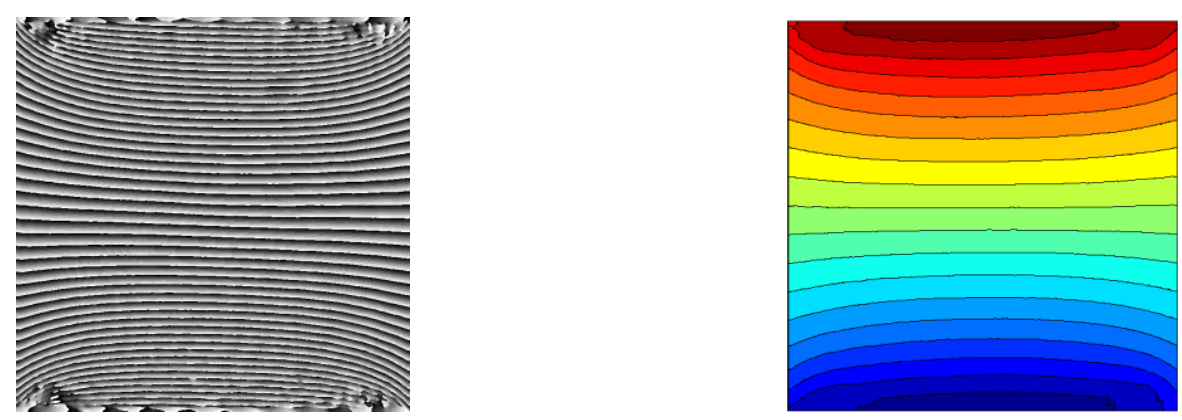

Figure 4-16: Fringe pattern and refractive index, run 38

This Run has the lowest Rayleigh vibration number among other selected Runs. The trend of $\Delta \mathrm{C}$ clearly shows higher separation in comparison with other Runs from (50\% IPA-50\% Water) mixture

\subsubsection{Analysis of Run 32 Results}

The vibration and temperature that are applied in Run 32 are as follow: This run has 
frequency of $0.5 \mathrm{~Hz}$ and amplitude of $0.07 \mathrm{~m}$ which gives Rayleigh number of 908.11 based on equation 2-1. Maximum value of temperature difference is applied in this run. The temperature difference between the top and bottom plate of the cell is $(\Delta \mathrm{T}=15 \mathrm{~K})$ which is assumed to be uniformly distributed in the experimental cavity. Figure 4-17 shows the concentration profile in the direction of temperature gradient versus the duration time of the experiment.

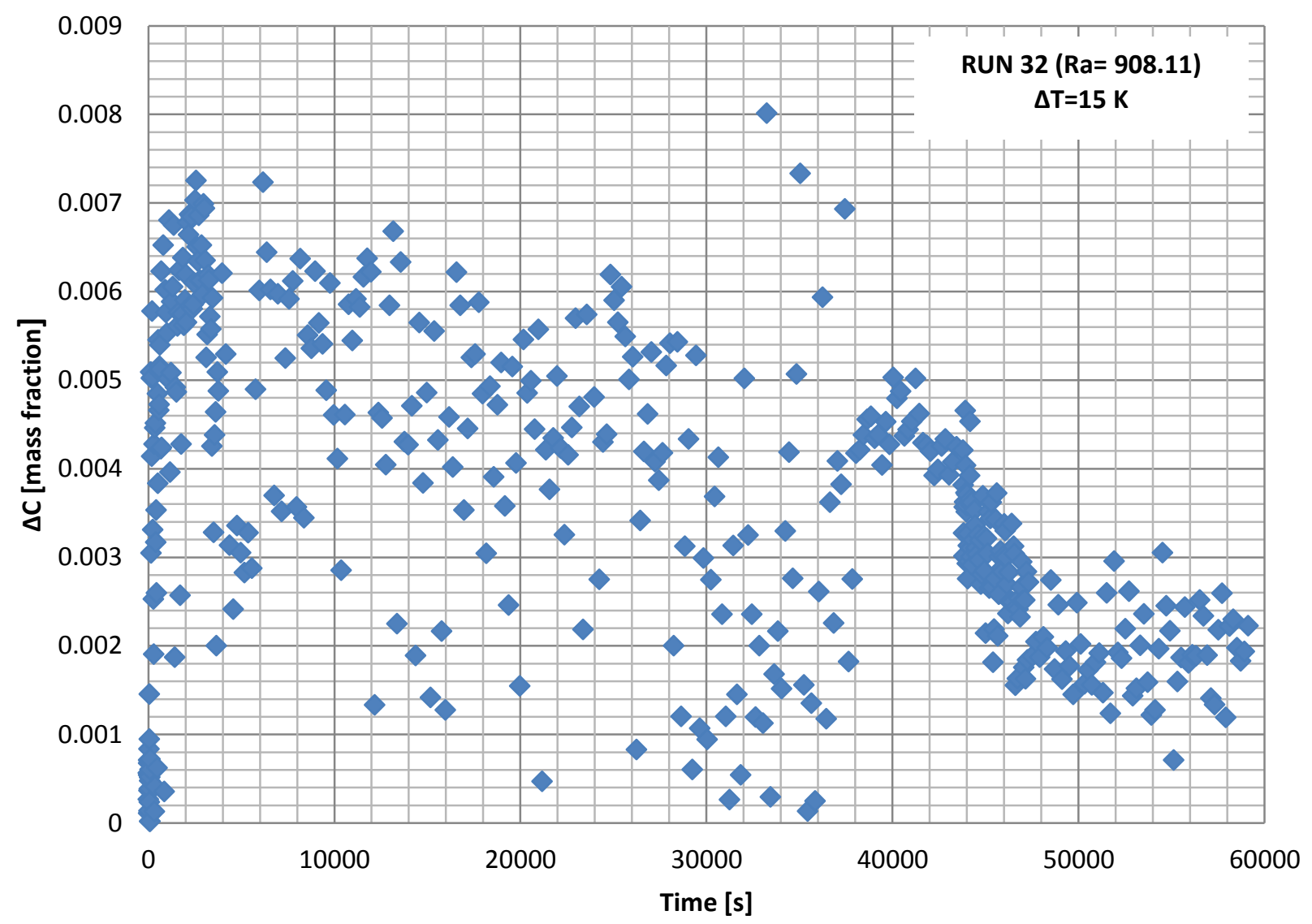

Figure 4-17: Concentration profile for Run 32

As it can be seen from Figure 4-17, very scattered results are obtained through image processing of run 32 data. This run has the same frequency and amplitude as run 34 and run 36 but it has the maximum temperature difference which is $15 \mathrm{~K}$ according to Table $4-2$. Hence, the effect of temperature gradient applied to the mixture can be shown by comparing these runs. 
However, drawing any conclusion based on these results is very difficult especially when dealing with positive Soret coefficient. Not only the system is already unstable but also the temperature difference is high. Figure 4-18 shows fringe pattern in an image chosen right after temperature is stabilized in the cavity. Very distorted fringes are seen at the top and bottom of the cavity. The noise in the fringe pattern are more compare to run 36 and Run 38 in which $\Delta \mathrm{T}=10 \mathrm{~K}$.
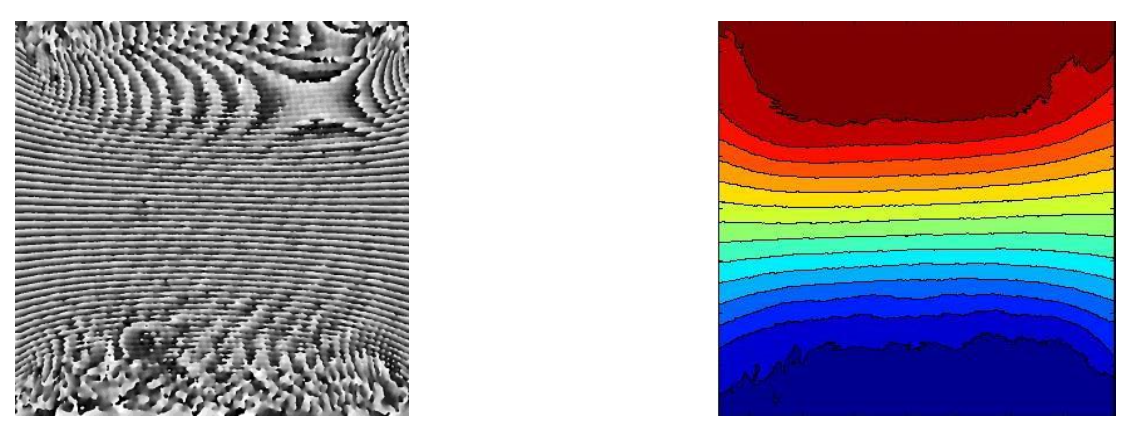

Figure 4-18: Fringe pattern and refractive index, run 32

It should be noted that unwrapping process require fringe pattern without or limited amount of noise since every fringe belongs to range of $\pi$ to $-\pi$ and existence of such distorted boundaries have negative impact on processed results.

Nonetheless, drawback of a built in program (this particular technique) are clearly shown in the case of high temperature difference. Results are very scattered during thermodiffusion stage. However, the maximum $\Delta \mathrm{C}$ is chosen to calculate the steady state Soret coefficient.

Lack of literature information and experimental measurements on positive Soret separation make any validation difficult. Further, more studies need to be done with repeated runs and high temperature difference to find out whether the cause of the issues is the data processing or the experimental procedure. Moreover, applying the highest vibration with $\mathrm{Ra}=908.11$ not only reduces the separation but also enhances instability in the system which add to other cause of awkward pattern of concentration change in cavity. 
The influence of vibration is noticeable when comparing all runs together. Table 4-2 shows steady state Soret separation obtained from maximum concentration difference in the system.

\begin{tabular}{|c|c|c|c|c|c|c|}
\hline $\mathbf{R U N} \#$ & $\mathbf{f}[\mathbf{H z}]$ & $\mathbf{A}[\mathbf{m}]$ & $\boldsymbol{\Delta} \mathbf{T}(\mathbf{2 5} \pm \mathbf{\Delta} \mathbf{T} / \mathbf{2})$ & $\mathbf{R a}_{\text {vibration }}$ & $\boldsymbol{\Delta C}_{\text {Steady }}$ & $\mathbf{S}_{\mathbf{T}, \mathbf{S t e a d y}}[\mathbf{1} / \mathbf{K}]$ \\
\hline $\mathbf{3 2}$ & 0.5 & 0.07 & 15 & 908.11 & $5.25 \times 10^{-3}$ & $1.40 \times 10^{-3}$ \\
\hline $\mathbf{3 4}$ & 0.5 & 0.07 & 5 & 100.90 & $2.79 \times 10^{-3}$ & $2.23 \times 10^{-3}$ \\
\hline $\mathbf{3 6}$ & 0.5 & 0.07 & 10 & 403.60 & $5.55 \times 10^{-3}$ & $2.19 \times 10^{-3}$ \\
\hline $\mathbf{3 8}$ & 0.1 & 0.07 & 10 & 16.14 & $1.34 \times 10^{-2}$ & $5.38 \times 10^{-3}$ \\
\hline
\end{tabular}

Table 4-2: Comparison of $\Delta C$ and $S_{T}$ for ISS experimental runs at steady state condition

The effect of vibration on separation ratio is clearly shown based on the above table. Although, the behaviour of mixture is questionable, as the Rayleigh vibration number increases separation decreases. This will indicate that vibration has negative impact on accuracy of thermodiffusion measurement on board ISS.

As it has been mentioned, there is not enough experimental information and benchmarks with regard to mixtures with positive Soret coefficient. However, based on recent study by Mialdun et al. [53] benchmark value for a run with zero force vibration and temperature difference of $15 \mathrm{~K}$ is $S_{T}=5.87 \times 10^{-3} \mathrm{~K}^{-1}$. Run 38 has the lowest vibration witch has the steady state Soret coefficient $\left(S_{T}=5.38 \times 10^{-3} K^{-1}\right)$. There is a close agreement between the benchmark and the obtained value that illustrates the accuracy of the results. 


\section{CHAPTER 5 CONCLUSION AND RECOMMENDATIONS}

\subsection{Conclusion}

A novel experimental approach based on optical digital interferometry (i.e. IVIDIL) is used to show the impact of forced vibration on thermodiffusion measurement.

There is no doubt that microgravity provides a suitable environment for conducting thermodiffusion measurements where they can be benefiting from weightlessness condition. However, the benefits of microgravity environment could be altered by g-jitter or unavoidable micro-accelerations onboard space platforms. Very small disturbances due to buoyancy driven flows can highly impact the results.

The aim of this research is achieved by determining the impacts of varying selected forced vibration level within different temperature gradients on thermodiffusion measurement. For this purpose, eight different runs of water and Isopropanol with both negative and positive Soret coefficient are tested under the microgravity condition. Digital images are recorded at a selected rate during the observation of heat and mass transfer. The

images give information about changes in the refractive index. Mathematical analysis in means of Fast Fourier Transform image processing is employed to obtain the results. In turn, it defines the local gradients of composition inside the fluid. In other words, the concentration difference as a result of thermodiffusion phenomenon is achieved in a steady state condition. Obtained results are divided in the two groups of 10\% IPA-90\% water mixture (negative Soret coefficient) and 50\% IPA $-50 \%$ water mixture (positive Soret coefficient). The latter goes through further discussion as this mixture tends to be unstable under higher thermal gradient.

The results for the mixture with negative Soret coefficient are compared with the case without any force or g-jitter vibration. Results clearly indicate the influence of difference vibration level on the thermodiffusion measurement in the test mixture. Vibration causes convection and remixes the fluid mixture resulting in imperfect development of Soret 
separation. Also, having non-uniform temperature can be a source of convection in the system. The calculated Soret coefficient from image processing is in good agreement with the benchmark value. Nonetheless, the effect of vibration is clearer during thermodiffusion time when separation is due to Soret effect. Results specify a possible relationship between separation and frequency, and between separation and amplitude of the vibration. Also, based on the results less separation appears within Isopropanol and water components as Rayleigh vibration number increases. In short, results show maximum separation for the case with minimum Rayleigh vibration number; however, linear relation between Rayleigh vibration and maximum separation cannot be detected.

Results that are obtained from analysing the mixture with positive Soret coefficient show significant variation from the mixture with negative Soret coefficient. There is noticeable instability in the concentration field while dealing with positive Soret coefficient. In a binary mixture, the lighter component with positive separation ratio migrates to the hot wall of the experimental cavity, which augments thermal buoyancy enhancing instability in the system. However, results illustrate similar relation between the forced vibration and separation of components as the case with negative Soret coefficient. Nonetheless, drawing an exact conclusion is difficult because not only the system is unstable but also high temperature difference is applied in the second sets of experimental runs. 


\subsection{Limitations}

There are some limitations and errors influencing the results of this study. The available time for a space experiment is a limitation of IVIDIL experiment. The duration of experiment is a compromise between relaxation time of the mixture and the available microgravity time. Some of the runs from the second sets with positive Soret coefficient need more time to reach steady state condition. Also, an error may be caused by the improper design of the cell that lead to appearance of heat flux and undesirable thermal gradient. As a result convection appears at the lateral walls of the cell and in the corners. This problem discards the advantages of Interferometer technique by disturbing the experiment.

It should be noted that there are often additional unknown sources of error beyond the statistical and systematic errors. These errors might be too complex and difficult to quantify. They can be caused by unwanted and minor experimental details such as nonuniform boundary conditions, small temperature inhomogeneity, or stability problems.

Furthermore, lack of literature and experimental studies, limited number of available data, and incompatibility of image processing method add to limitation of analysing the data. 


\subsection{Recommendations}

This study has focused on the analysis of selected low Rayleigh vibration IVIDIL runs to illustrate the effect of g-jitter on thermodiffusion measurement of binary mixtures in microgravity environment. Therefore, some recommendations can be made based on the outcomes of this research that may be used as continuation of this study.

This study can be done for multi-component mixtures to clearly illustrate the importance of thermodiffusion applications. It should be noted that research groups are working on the extended IVIDIL experiments to fulfil this purpose. Also, experimental runs involving stronger Rayleigh vibration can be analysed along with some repeatability in the experiments with different remixing approach and longer duration. This suggestion might improve the understanding of observed unknown instability in the positive Soret coefficient mixture. Moreover, a more advanced image processing technique can be used to be compatible with positive Soret coefficient and higher temperature distribution. The method should solve the problem in transient state using curve fitting and special algorithm that provide an optimum solution to the problem. The constraints and errors of the current technique could be minimized when eliminating noises of the images and extract real trends and patterns. 


\section{PUBLICATIONS}

- A. Kianian, A. Ahadi, Z. Saghir, Experimental Evidence of Low Rayleigh Vibration on Mixture during Thermodiffusion Experiment, The Canadian Journal of Chemical Engineering (2012) 9999.

- Ahadi, A. Kianian, Z. Saghir, Heat and Mass Transport Phenomena under Influence of Vibration Using a New Aided Image Processing Approach, International Journal of Thermal Science 


\section{APPENDICES}

\section{Appendix A: MATLAB code to generate phase difference}

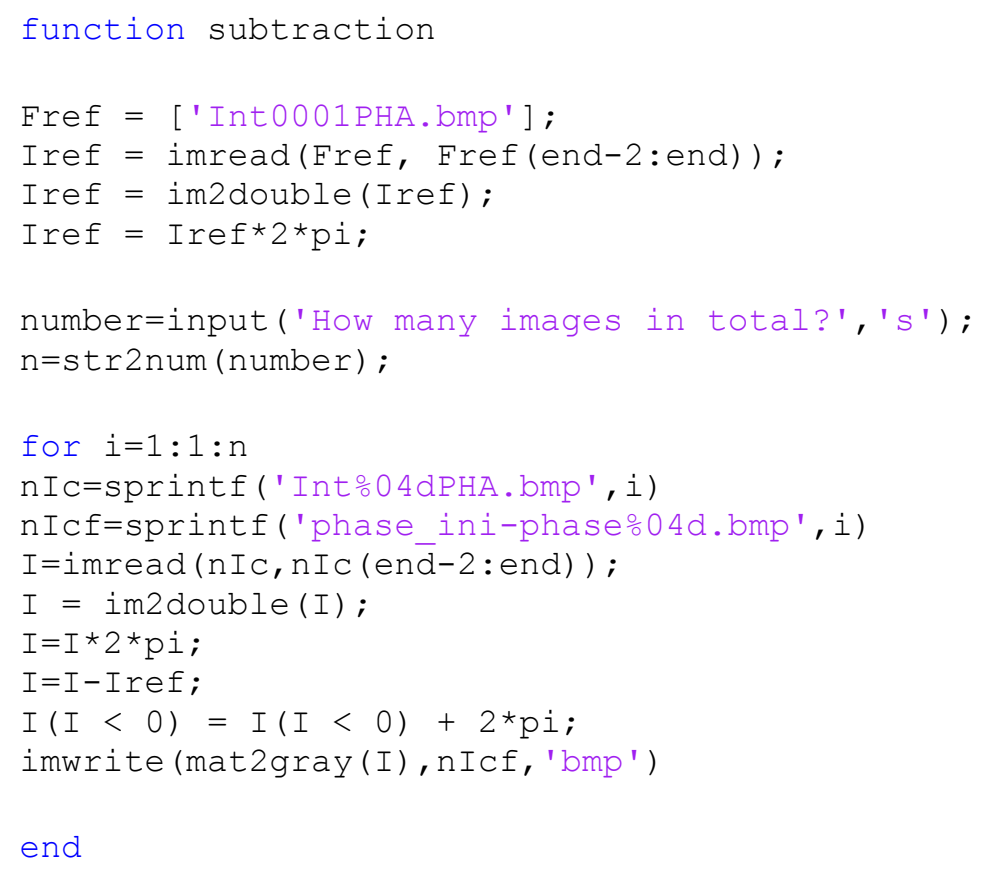




\section{Appendix B: MATLAB code to generate concentration difference}

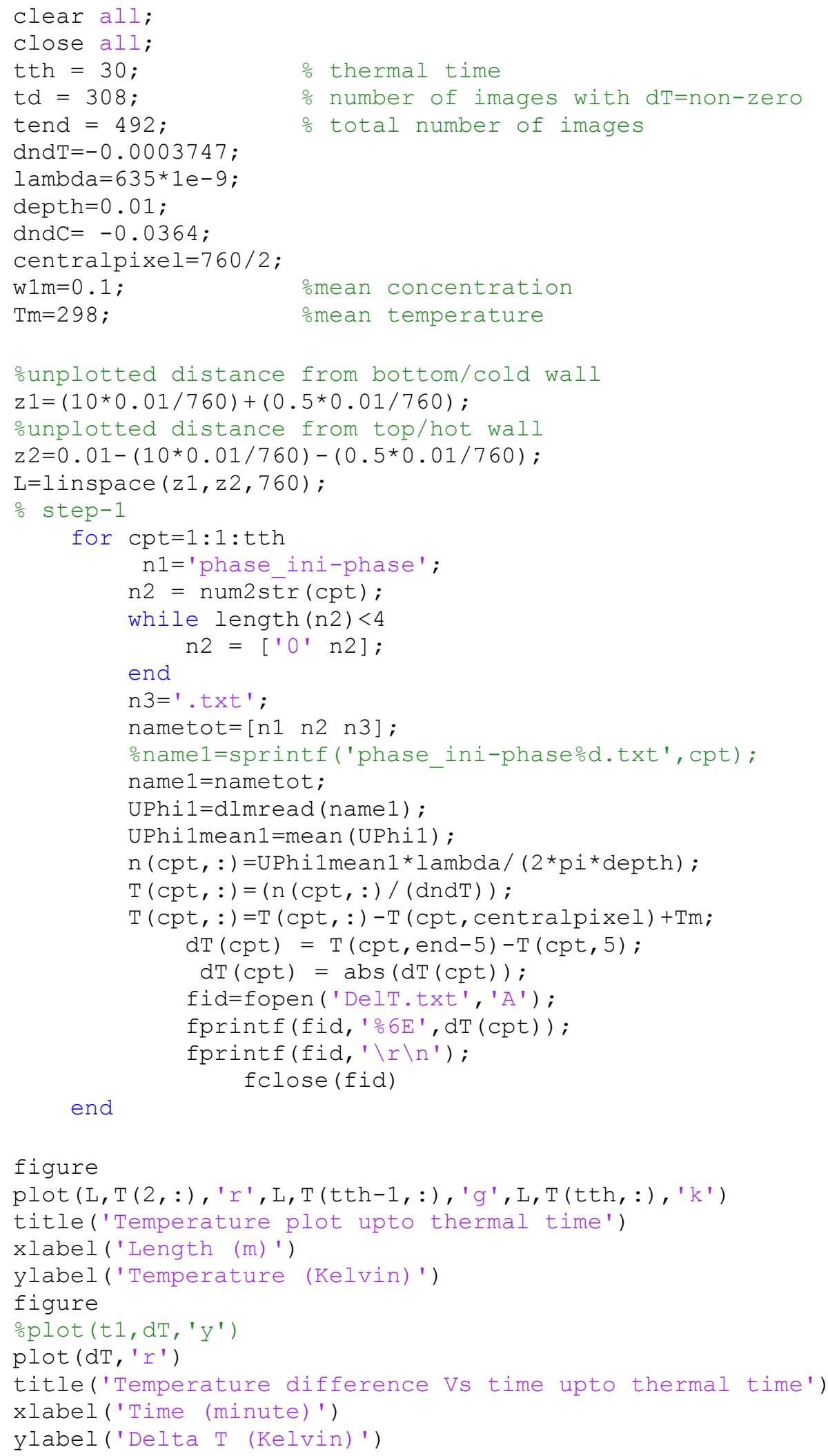




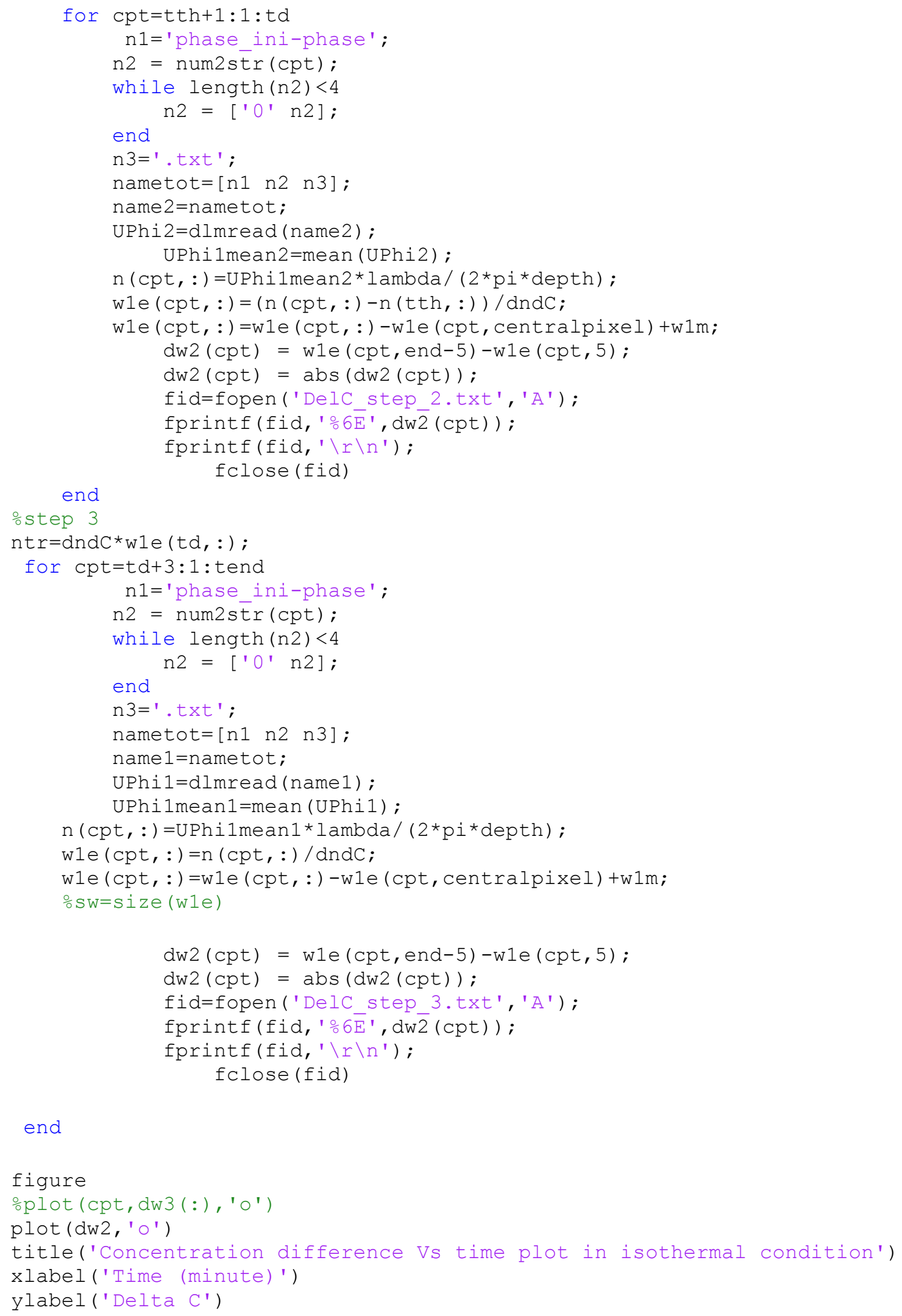




\section{BIBLIOGRAPHY}

[1] J.K. Platten, The Soret effect: a review of recent experimental results, Journal of Applied Mechanics 73 (2006) 5-13

[2] M. Touzet, G. Galliero, V. Lazzeri, M.Z. Saghir, F. Montel, J.C. Legros, Thermodifusion: from microgravity to the initial state of petroleum reservoirs, Journal of C.R. Mecanique 339 (2011) 318-323

[3] L.J.T.M. Kempers, A comprehensive thermodynamic theory of the Soret effect in a multicomponent gas, liquid, or solid, Journal of Chemical Physics 115 (2001) 63306341

[4] Eckert, E. R. G., Carlson, W. O., Natural convection in a square cavity, International Journal of Heat and Mass Transfer, 2 (1961) 106-120

[5] M. Chacha, D. Faruque, M.Z. Saghir, J.C. Legros, Solutal thermodiffusion in binary mixture in the presence of g-jitter, International Journal of Thermal Sciences. 41 (2002) 899-911.

[6] S. Srinivasan, M.Z. Saghir, Experimental approaches to study thermodiffusion- a review, International Journal of Thermal Sciences 50 (2011) 1125-1137

[7] S. Pan, Y. Yan, T. J. Jaber, M. Kawaji, M.Z. Saghir, Evaluation of thermal diffusion models for ternary hydrocarbon mixtures, Journal of Non-Equilibrium Thermodynamics 32 (2007) 241-249

[8] M. Eslamian, M.Z. Saghir, M. M. Bou-Ali, Investigation of the Soret effect in binary, ternary and quaternary hydrocarbon mixtures: New expressions for thermodiffusion factors in quaternary mixtures, International Journal of Thermal Sciences 49 (2010) 2128-2137

[9] S. Srinivasan, M.Z. Saghir, Measurements on thermodiffusion in ternary hydrocarbon mixtures at high pressure, Journal of Chemical Physics 131 (2009) 124508

[10] F. Montel, J. Bickert, A. Lagisquet, G. Galliero, Initial state of petroleum reservoirs: a comprehensive approach, Journal of Petroleum Science and Engineering 58 (3-4) (2007) 391-402 
[11] E.A. Spiegel, Convection in stars-II: special effects, Annual Review of Astronomy and Astrophysics 10 (1972) 261-304

[12] M. Gregg, The microstructure of the ocean, Scientific American 228 (1973) 65-77

[13] M. Eslamian, M.Z. Saghir, A critical review of thermodiffusion models: role and significance of the heat of transport and the activation energy of viscous flow, Journal of Chemical Physics 127 (2007) 14502

[14] S.Wiegand, Thermal diffusion in liquid mixtures and polymer solutions, Journal of Physics: Condensed Matter 16(2004) R357-R379

[15] P. Costeséque, T. Pollak, J.K. Platen, M. Marcoux, Transient state method for coupled evaluation of Soret and Fick coefficients, and related tortuosity factors, using free and porous packed thermodiffuison cells: application to $\mathrm{CuSO}_{4}$ aqueous solution (0.25 M), European Biophysical Journal 15 (3) (2004) 249-253

[16] B.D. Butler, J.C.R. Turner, Flow-cell studies of thermal diffusion in liquids. Part 1cell construction and calibration, Transactions of the Faraday Society 62 (1966) 3114-3120

[17] D. Longree, J.C. Legros, G. Thomaes, Measured Soret coefficients for simple liquefied gas mixtures at low temperatures, Journal of Physical Chemistry 84 (1980) 3480-3483

[18] M.M. Bou-Ali, O. Ecennaro, J.A. Madariaga, C.M. Santamaria, J.J. Valencia, Stability of convection in a vertical binary fluid layer with an adverse density gradient, Physical Review E 59 (1999a) 1250-1252

[19] J.F. Dutrieux, j.K. Platten, G. Chavepeyer, M.M. Bour-Ali, On the measurements of positive Soret coefficients, Journal of Physical Chemistry 106 (23) (2002) 61046114

[20] J.K. Platten, M.M. Bou-Ali, J. Durieux, Enhanced molecular separation in inclined thermogravitational columns, Journal of Physical Chemistry B 107 (42) (2003) 11763-11767

[21] P.Kolodner, H. Williams, C. Moe, Optical measurement of the Soret coefficient of ethanol/water solutions, Journal of Chemical Physics 88 (1988) 6512-6524

[22] P.A. Artila, B. Rousseau, Microscopic interpretation of a pure chemical contribution to the Soret effect, Physical Review Letters 98 (2007) 125901 
[23] S.A. Putnam, D.G. Cahill, Micron-scale apparatus for measurements of thermodiffusion in liquids, Review of Scientific Instruments 75 (7) (2004) 23682372

[24] A. Perronace, C. Leppla, F. Leroy, B. Rousseau, S. Wiegand, Soret and mass diffusion measurements and molecular dynamics simulations of n-pentane-n-decane mixtures, Journal of Chemical Physics 116 (2002) 3718

[25] W. Köhler, Thermodiffusion in polymer solutions as observed by forced Rayleigh scattering, Journal of Chemical Physics 98 (1) (1993) 660-668

[26] H. Ning, R. Kita, H. Kriegs, J.Luettmer-Strathmann, S. Wiegand, Thermal diffusion behavior of nonionic surfactants in water, Journal of Physical Chemistry B 110 (2006) 10746

[27] V. Shevtsova, A. Mialdun, D. Melnikov, I. Ryzhkov, Y. Gaponenko, Z. Saghir, T. Lyubimova, J.C. Legros, IVIDIL experiment onboard ISS: thermodiffusion in presence of controlled vibrations, Comptes Rendus Mecanique, C. R. Mecanique 339 (2011) 310-317

[28] A. Mialdun, D. Melnikov, V. Shevtsova, 2006, Observation of Diffusion Phenomena in Ground Experiments: Problems and Solutions, in Proceeding of IAC Conference, Valensia, Spain, 12p. IAC-06-A2-4-05

[29] R. Monti (2001). Physics of fluid in microgravity, London: Taylor \& Francis

[30] V. Shevtsova, A. Mialdun, D. Melnikov, I. Ryzhkov, Yu. Gaponenko, Z. Saghir, T. Lyubimova and J.C. Legros, 2010, IVIDIL Experiment Onboard ISS, in Proceeding IAC Conference, Sept. 27 - Oct. 01, 2010, Prague, Czech, IAC-10-A2-6-09

[31] M. Sakurai, N. Ohishi, A. Hirata, Oscillatory thermocapillary convection features in a liquid bridge under normal gravity and microgravity conditions-Drop shaft experiments, Advances in Space Research. 24 (1999) 1379-1384.

[32] T. Carlberg, Floating zone experiments with germanium crystals in sounding rockets, Acta Astronaut. 13 (1986) 639-643.

[33] V. Pletser, Short duration microgravity experiments in physical and life sciences during parabolic flights: the first 30 ESA campaigns, Acta Astronaut. 55 (2004) 829-854. 
[34] R. Roşu-Pflumm, W. Wendl, G. Müller-Vogt, S. Suzuki, K.-. Kraatz, G. Frohberg, Diffusion measurements using the shear cell technique: Investigation of the role of Marangoni convection by pre-flight experiments on the ground and during the Foton M2 mission, Int. J. Heat Mass Transfer. 52 (2009) 6042-6049.

[35] R. Savino, D. Paterna, Compatibility of the microgravity environment of the international space station with fluid and material science experimentation, Acta Astronaut. 51 (2002) 229-241.

[36] P. Baglioni, R. Demets, A. Verga, ESA payloads and experiments on the Foton-12 mission. (2000).

[37] S. Mazzoni, V. Shevtsova, A. Mialdun, D. Melnikov, Y. Gaponenko, T. Lyubimova, M.Z. Saghir, Vibrating liquids in space, Europhys. News. 41 (2010) 14-16.

[38] G. F. Putin, L. A. Babushkin, G. P. Bogatyrev, A. F. Glukhov, E. A. Zilberman, A. I. Ivanov, M. M. Maksimova, V. I. Polezhaev, O. A. Bessonov, S. A. Nikitin, V. V. Sazonov, On the Measurement of Low-Frequency Microaccelerations on Board an Orbital Station by the Convection Sensor "DACON", Journal of Advances in Space Research 32 (2003) 199-204

[39] J. P. Garandet, J. I. D. Alexander, S. Corre, J.J. Favier, Composition variations induced by g-jitter in Bridgman growth of Sn-Bi alloys in microgravity, Journal of Crystal Growth 226 (2001) 543-554

[40] Smith R. W., Zhu X., Tunnicliffe M. C., Smith T. J. N., Misener L, Adamson J., The measuremner of precise solute diffusion coefficients in dilute liquid metals and metalloids in a low earth orbiting laboratory - the influence of g-jitter, European Space Agency, (Special Publication), 454 (2000) 887-893

[41] M. Shirkhanzadeh, Analysis of the data obtained from liquid metal diffusion experiments conducted on the MIR space station, Journal of Acta Astronautica, 64 (2009) 256-263

[42] A.H. Ahadi, M.Z. Saghir, Quasi Steady State Effect of Micro Vibration from Two Space Vehicles on Mixture During Thermodiffusion Experiment, FDMP; Fluid Dynamics and Material Processing. 287 (2012). 
[43] V. Shevtsova, D. Melnikov, J. C. Legros, Y. Yan, Z. Saghir, T. Lyubimova, G. Sedelnikon, B. Roux, Influence of vibrations on thermodiffusion in binary mixture: A benchmark of numerical solutions, Journal of Physics of Fluids, 19 (2007) 017111

[44] S. Srinivasan, M. Dejmek, M.Z. Saghir, Thermo-solutal-diffusion in high pressure liquid mixtures in the presence of micro-vibrations, International Journal of Thermal Sciences. 49 (2010) 1613.

[45] V. Shevtsova, T. Lyubimova, Z. Saghir, D. Melnikov, Y. Gaponenko, V. Sechenyh, J.C. Legros, A. Mialdun, IVIDIL: on-board g-jitters and diffusion controlled phenomena, International Symposium on Physical Sciences in Space. 327 (2011) 012031-012031.

[46] A. Ahadi, A. Kianian, M.Z. Saghir, Heat and Mass Transport Phenomena under Influence of Vibration Using a New Aided Image Processing Approach, International Journal of Thermal Science, (Submitted).

[47] A. Mialdun, V. Shevtsova, Open Questions on Reliable Measurements of Soret Coefficients, Journal of Microgravity Science and Technology, 21 (2009) 31-36

[48] A. Parsa, S. Srinivasan, M.Z. Saghir, Themodiffusion of Binary Fluid Mixture in The Reduced Gravity Environment of The International Space Station, International Astronautical Congress 2010, IAC 2010, v 11, p 9203-9206

[49] E. Leussink, Selectable Optical Diagnostics Instrument (SODI) Software User Manual, SODI-MAN-372-VE Rev. 1, Verhaert Space Company (2009) 26-29.

[50] M. Hipp, J. Woisetschlager, P. Reiterer, T. Neger, Digital evaluation of interferograms, Journal of Measurement 36 (2004) 53-66

[51] A. Mialdun, V. Shevtsova, Measurement of the Soret and diffusion coefficients for benchmark binary mixtures by means of digital interferometry, Journal of Chemical Physics 134 (2011) 044524

[52] A. Mialdun, V. Shevtsova, Development of optical digital interferometry technique for measurement of thermodiffusion coefficients, International Journal of Heat and Mass Transfer 51(2008) 3164-3178

[53] A. Mialdun, V. Yasnou, V. Shevtsova,, A. Koniger, W. Kohler, D. Alonso de Mezquia, M. M. Bou-Ali, A comprehensive study of diffusion, thermodiffusion, 
and Soret coefficient of water-isopropanol mixture, Journal of Chemical Physics, 136 (2012) 244512

[54] V. Shevtsova, I. I. Ryzhkov, D. E. Melnikov, Y. A. Gaponenko, A. Mialdun, Experimental and theoretical study of vibration-induced thermal convection in low gravity, Journal of Fluid Mechanics 648 (2010) 53-82

[55] V. Shevtsova, D. E. Melnikov, J.C. Legros, Onset of convection in Soret-deriven instability, Journal of Physical Review, 73 (2006) 047302

[56] B. Huke, M. Lucke, Convection Patterns in Binary Fluid Mixtures with Positive Separation Ratios, Journal of Physics of Fluid, (2006) 151150

[57] S. Mazzoni, V. Shevtsova, A. Mialdun, D. Melnikov, Yu. Gaponenko, T. Lyubimova, M. Z. Saghir, Vibrating Liquids in Space, Euro Physics News, DOI: 10.1051/epn/2010601 